\title{
Berechnung gewölbter Platten
}

\section{Doctoral Thesis}

\section{Author(s):}

Keller, Huldreich

Publication date:

1912

Permanent link:

https://doi.org/10.3929/ethz-a-000091960

Rights / license:

In Copyright - Non-Commercial Use Permitted 


\title{
Berechnung gewölbter Platten.
}

\author{
Von der \\ Eidgenössischen Technischen Hochschule in Zürieh \\ zur Erlangung der
}

Würde eines Doktors der technischen Wissenschaften

genehmigte

Promotionsarbeit

vorgelegt von

Huldreich Keller, Dipl.=Ing.

aus Arbon (Schweiz).

Referent: Herr Professor Dr. E. Meissner.

Korreferent: Herr Professor Dr. A. Stodola.

Berlin 1912. 
Der Verfasser vorliegender Arbeit wurde geboren am 16. März 1868 in A r b o n am Bodensee. Ich besuchte während sechs Jahren die Primar- und sodann während drei Jahren die Sekundarschule meines Geburtsortes und im Anschluss daran dreieinhalb Jahre hindurch die technische Abteilung der thurgauischen Kantonsschule in F r a u e $\mathrm{n} f \mathrm{e} l \mathrm{~d}$.

Alsdann machte ich drei Jahre Werkstattpraxis teils in den Werkstätten meines Vaters, teils in den Maschinen-Fabriken von $\mathrm{B}$ a $\mathrm{um}$ und von $\mathrm{S}$ a $\mathrm{u}$ r e $\mathrm{r}$ in Arbon.

Vom Oktober 1889 bis März 1893 besuchte ich die mech. technische Abteilung des eidgenössischen Polytechnikums in Zürich und erhielt daselbst zum Schluss das Diplom eines Maschineningenieurs. Während des darauffolgenden Jahres war es mir vergönnt, eine Assistentenstelle bei Herrn Professor Stodola einzunehmen. Sodann bekleidete ich die Stelle eines Konstruktionsingenieurs in der Dampfmaschinen-Abteilung von Escher Wyss \& Cie. in Zürich. Im Sommer-Semester 1895 war ich aushilfsweise als Lehrer am Technikum Winterthur verpflichtet.

Vom Herbst 1896 bis Ende 1908 war ich bei der AE G Berlin engagiert und zwar etwa sieben Jahre lang in der Maschinenfabrik Brunnenstrasse und fünf Jahre lang in der neugegründeten Turbinenfabrik Huttenstrașe. An beiden Orten war mir insbesondere das mechanische Laboratorium unterstellt. Zu Ende 1908 kehrte ich wieder zu Escher Wyss \& Cie. in Zürich zurück, wo ich seither dem Literarischen und dem Patentbureau vorstehe. 


\title{
Berechnung gewölbter Platten.
}

\author{
Von Ingenieur Huldreich Keller in Zürich.
}

Durch vorliegende Arbeit soll der Weg gezeigt werden für eine annäherungsweise Berechnung von gewölbten Platten. Er ist ähnlich demjenigen, den ich einer früheren Arbeit über die Berechnung von umlaufenden Rạdscheiben zugrunde gelegt habe ${ }^{1}$ ). Das Hauptkennzeichen dieses Rechnungsverfahrens liegt darin, daß man die Differentialgleichungen, anf die man gelangt, dureh das annäherungsweise »Rechnen mit kleinen Differenzen" löst.

Unsere neue Aufgabe ist aber wesentlich umfangreicher, als die Berechnung von Radscheiben, weil $z u$ den Normalspannungen in radialer und tangentialer Richtung, wie sie in Radscheiben fast allein vorkommen, in einer einseitig belasteten, gewölbten Platte noch Schub- und Biegungsspannungen hinzutreten.

Der 'Wweck vorliegender Arbeit soll insbesondere auch darin bestehen, die teils ziemlich verwickelten Formeln in eine möglichst einfache Form zu bringen, wie sie für ein am Konstruktionstisch gefordertes, hinreichend zuverlässiges Rechnen brauchbar ist, das nicht allzusehr ermüdet.

Die Berechnung soll die Möglichkeit schaffen, in einer als Drehkörper durchgebildeten, gewölbten (oder ebenen) Platte, welche von einer Seite durch einen Gas- oder Fluissigkeitsdruck belastet ist, in jedem Punkt die Beanspruchung und die Formländerung zu ermitteln. Es kommen also Platten in Frage, wie sie als Deckel und Zwischenböden in Dampitturbinen, als Böden von Dampfkesseln oder andern Hochdruckgefí Ben Verwendung finden.

Das Verfahren setzt voraus, daß die Dicke der Platte im Verhältnis zum Krümmungshalbmesser des Meridians klein, der Stoff durchaus homogen und $\mathrm{d} \boldsymbol{Q} ß$ jede schroffe Querschnittänderung vermieden sei. Ferner soll die Forminderung entsprechend der für den gebogenen Balken aufgestellten Bernoullischen Annahme derart vor sich gehen, daß alle Punkte der Platte, welche vor der Biegung auf einer zur Plattenwölbung senkrechten Geraden lagen, auch nach der Durchbiegung auf einer Geraden liegen, die senkrecht steht zur MittelHläche ${ }^{2}$.

In die Rechnung führen wir folgende, aus Fig. I ersichtliche Bezeichnungen ein:

i) Slehe -Schweizerische Bauzeitung; vom 27. November I909 S. 307 und 》Die Turbine vom 5. Dezember 1909 S. 88.

2) Dies trifft für guBeiserne gewölbte Platten nur annïherungsweise zu. Vergl. Bach, "Elastizitat und Festigkeit» I902 S. 504 u. f. über »gekrünımte, stabfürmige Korper๙.

Diss, Keller. 
$x$ in cm Abstand des auf der Meridian-Mittelfaser $\widehat{J A}_{1}$ gelegenen zu untersuchenden Punktes $A$ von der Symmetrieachse $z-z$ der Platte.

$h$ in cm Dicke der Platte beim Punkt $A$.

$\eta$ in $\mathrm{cm}$ Abstand eines auf dem durch $A$ gehenden Krümmungshalbmesser des Meridians gelegenen Punktes $C$ von $A$.

0 in $\mathrm{cm}$ Krümmungshalbmesser der Meridian-Mittelfaser im Punkt $A$.

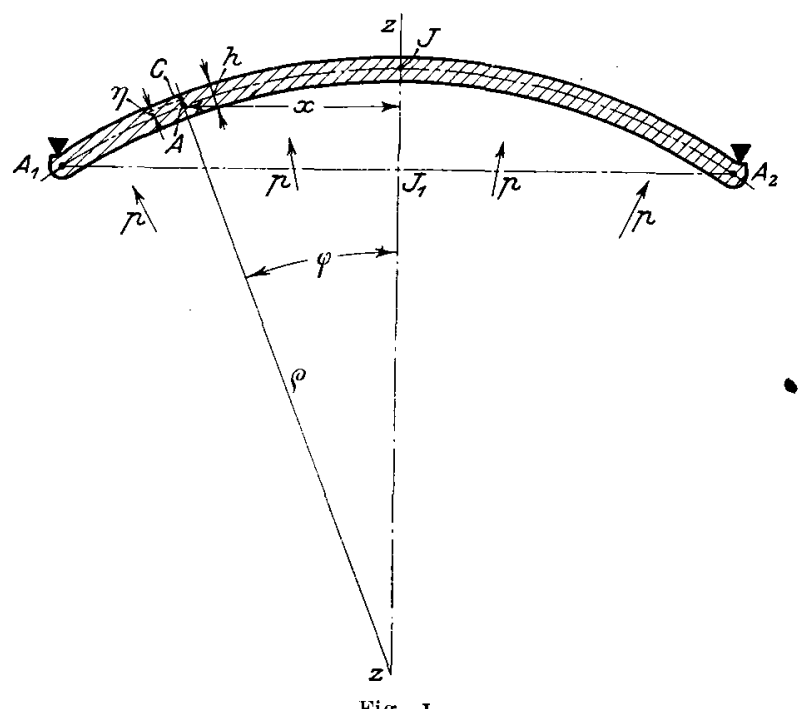

$\Phi$ Winkel zwischen diesem Krümmungshalbmesser und der Symmetrieachse.

$\Delta \varphi \equiv \psi$ Aenderung dieses Winkels $\varphi$ bei der Durchbiegung.

$\Delta(d q) \equiv d \psi$ Aenderung des Winkelelementes $d q$.

$\infty=\frac{\Delta(d \varphi)}{d \varphi}$ spezifische Aenderung des Winkelelementes.

$p$ in $\mathrm{kg} / \mathrm{qcm}$ der gleichmäßig verteilte, einseitige Ueberdruck, winkelrecht auf die Platte wirkend. Für eine Einzelbelastung $P$ wären diejenigen Glieder der Rechnung, in denen sonst $p$ vorkommt, sinngemäß zu ändern. Ein Gleiches gilt für ein Zusammenwirken einer gleichmäßig verteilten Be lastung $p$ und einer Finzellast $P$.

$\sigma_{r}$ in $\mathrm{kg} / \mathrm{qcm}$ die Normalspannung in einem den Punkt $C$ enthaltenden Flachenelement, das auf dem durch den Punkt $A$ oder $C$ senkrecht zur Plattenwölbung geführten Kegel liegt, dessen Symmetrieachse mit derjenigen der Platte zusammenfallt. $\sigma_{r}$ ist gleich gerichtet wie die Meridian-Mittelfaser. Der Hauptsache nach verläuft sie »radial«; ihr Wert sei deshalb abkürzungshalber mit »Radialspannung " bezeichnet -, dies in Anlehnung an die Scheibenberechnung.

$\sigma_{t}$ in $\mathrm{kg} / \mathrm{qcm}$ die Normalspannung (Hauptspannung) im Meridianschnitt; sie verläuft tangential zum Parallelkreis und soll deshalb »Tangentialspannung* heißen.

$\tau$ in $\mathrm{kg} / \mathrm{qcm}$ die Schubspannung in dem Flächenelement, auf welches $\sigma_{r}$ winkelrecht wirkt.

$E$ in $\mathrm{kg} / \mathrm{qcm}$ der Elastizitätsmodul des Plattenstoffes.

In Fig. I ist ein Meridianschnitt durch einen von der konkaven Seite, also in positiven Sinn mit der spezifischen Pressung $p$ belasteten Deckel gezeichnet. 
Durch diese Belastung $p$ erfährt ein im Abstand $x$ von der Symmetrieachse $z-z$ gelegenes Element der im Meridianschnitt liegenden Mittelfaser in Richtung dieser Faser die spezifische Verlängerung $\varepsilon_{r 0}$, ein im Abstand $\eta$ von der Mittelfaser liegendes, parallel $\mathrm{zu}$ ersterem gerichtetes Faserteilchen die spezifische Verlängerung $\varepsilon_{r}$. Diese Verlängerungen werden bedingt durch die an diesen Stellen herrschenden Radialspannungen $\sigma_{r 0}$ in der Mittelfaser und $\sigma_{r}$ im Abstand $\eta$ hiervon und den Tangentialspannungen $\sigma_{t 0}$ und $\sigma_{t}$.

\section{1) Berechnung der spezifischen Verlängerung $\varepsilon_{r}$.}

Die oben gemachte Annahme, daß die Plattendicke gering sei im Verhältnis zum Krümmungshalbmesser des Meridianschnittes, hat zur Folge, daß die spezifischen Dehnungen der Meridianfasern in einem linearen Verhältnis zu ihrem Abstand $y$ von der Mittelfaser angenommen werden können, gleich wie in einem gekrïmmten Balken ')

$$
\xi_{r}=\varepsilon_{r 0}+\left(\omega-\varepsilon_{r 0}\right) \frac{\frac{\eta}{\varrho}}{\mathrm{I}+\frac{\eta}{\varrho}} .
$$

Um die Rechnung zu vereinfachen und dadurch den praktischen Bedürfnissen anzupassen, wollen wir verschiedene Annäherungen zulassen. Weil die Dicke $h$ der Platte im Verhältnis zum Krümmungshalbmesser ! gering sein soll, dürien wir setzen:

$$
\varrho+\eta \sim \varrho ; \quad\left(\mathrm{I}+\frac{\eta}{\varrho}\right) \sim \mathrm{I} .
$$

Hierdurch vereinfacht sich die Gleichung für $\varepsilon_{r}$ auf

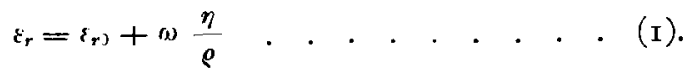

\section{2) Berechnung der spezifischen Verlängerung $\varepsilon_{t}$ im Parallelkreis vom Halbmesser $\xi$ und dem Abstand $\eta$ von der Mittelfaser.}

In Fig. 2 ist im vergrößerten Maße dargestellt, wie der Meridianschnitt aus seiner anfänglichen Lage $\overparen{A B}$ bei der Belastung in die Lage $\widehat{A}^{\prime} B^{\prime}$ verschoben wird. Dabei vergrößern sich die Halbmesser der durch den Mittenpunkt $A$ und den im Abstand " davon gelegenen Punkt $C$ gehenden Parallelkreise von den Anfangswerten $x$ und $\xi$ auf die Endwerte $(x+\Delta x)$ und $(\xi+J \xi)$. Hierbei erfährt der durch den Punkt $C$ gehende Parallelkreis eine spezifische Dehnung

$$
\varepsilon_{t}=\frac{2 \pi(\xi+\Delta \xi)-2 \pi \xi}{2 \pi \xi}=\frac{\Delta \xi}{\xi} .
$$

Nun ist gemäß Fig. 2

$$
\xi=x+\eta \sin \uparrow \text {. }
$$

Durch Differenzieren dieser Gleichung erhält man für die Zunahme von $气$ den Ausdruck

$$
\Delta \xi=\Delta x+\Delta \eta \sin \gamma+\eta \cos \eta \Delta q .
$$

\footnotetext{
1) Vergl. Bach, Elastizitäts- und Festigkeitslehre* 1902 S. 472.
}

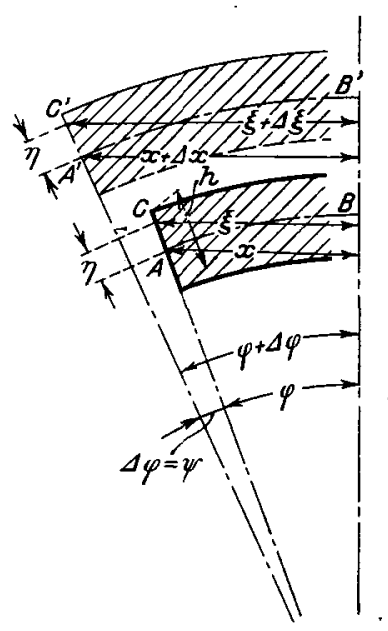

Fig. 2. 
Wegen der Kloinheit von $y$ und der daraus folgenden Kleinheit von $J y$ wird auf der rechten Seite der zweite Summand gegeniiber den beiclen anderen Summanden vermachlässigt, und es bleibt noch

$$
\Delta \xi=\Delta x+\eta \Delta \varphi \cos q .
$$

Diesen Wert eingesetzt in die Gleichung $\varepsilon_{t}=\frac{\Delta \xi}{\xi}$, gibt

$$
\varepsilon_{t}=\begin{gathered}
\Delta x+\eta \Delta \varphi \cos \varphi \\
x+\eta \sin \varphi
\end{gathered}
$$

Mit hinreichender Anniiherung kann man setzen $x+\eta \sin \varphi \sim x$; ferner ist $\frac{\Delta x}{x}=\varepsilon_{t 0}$, wo $\varepsilon_{t 0}$ die spezifische Dehnung des durch den Punkt $A$, d. h. im Abstand $\eta=0$ von der Mittelfaser gezogenen Pararallelkreises ist. Dadurch vereinfacht sich der Ausdruck für $\varepsilon_{t}$ auf die Form:

$$
\varepsilon_{t}=\varepsilon_{t 0}+{ }_{x}^{\eta} \Delta q \cos q \text {. . . . . . . . . . (2). }
$$

Hierin ist aber vorlaufig weder $\varepsilon_{t 0}$ noch $\lrcorner q$ bekannt.

\section{3) Berechnung der im Punkt $C$ herrschenden Radialspannung $\sigma_{r}$ und Tangentialspannung $\sigma_{i}$.}

Die Elastizitätslehre ${ }^{1}$ ) gibt zwischen den Spannungen und den Iehnungen die Beziehungen :

$$
\sigma_{r}=\frac{m E}{m^{2}-\mathrm{I}}\left[m \varepsilon_{r}+\varepsilon_{t}\right] ; \quad \sigma_{t}=\frac{m E}{m^{2}-\mathbf{I}}\left[\varepsilon_{r}+m \varepsilon_{t}\right]
$$

wo $m$ das Verhältnis der spezifischen Längsdehnung zur linearen Quer\%usammenziehung eines auf reinen Zug beanspruchten Stabes bedeutet ${ }^{2}$ ).

Wir setzen

$$
\text { c. } \quad \frac{m E}{m^{2}-\mathrm{I}} \cdot
$$

Unter Verwendung der Gl. (I) und (2) erhalten wir

$$
\begin{aligned}
& \sigma_{r}=c\left[m \varepsilon_{r 0}+m \omega \frac{\eta}{\varrho}+\varepsilon_{t 0}+{ }_{x}^{\eta} \Delta \varphi \cos \varphi\right] \\
& \sigma_{t}=c\left[\varepsilon_{r 0}+\omega \frac{\eta}{\varrho}+m \varepsilon_{t 0}+m \frac{\eta}{x} \Delta \varphi \cos q\right] .
\end{aligned}
$$

Setzen wir in diesen beiden Gleichungen $\eta=0$, so erhalten wir als Sonderfiille die Normalspannungen in Abstand $x$ von der Symmetrieachse und in der jeweiligen Mittelfaser des Meridianschnittes und des Parallelkreisschnittes:

$$
\begin{aligned}
& \sigma_{r 0}=c\left[m \varepsilon_{r 0}+\varepsilon_{t 0}\right] \\
& \sigma_{t 0}=c\left[\varepsilon_{r 0}+m \varepsilon_{t 0}\right] .
\end{aligned}
$$

Berücksichtigi man terner, daß

$$
\omega=\frac{\Delta d \varphi}{d \varphi} ; \quad \frac{\omega}{\varphi}=\frac{\Delta d \varphi}{\varphi d \varphi}={ }_{d s}^{d d \varphi} ; \quad d s=\underset{\cos \varphi}{d x} ; \quad \Delta d \varphi=d \psi
$$

so gehen die Gleichungen für $\sigma_{r}$ und $\sigma_{t}$ über in die Form

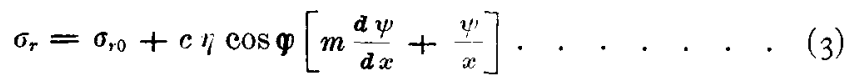

$$
\begin{aligned}
& \sigma_{t}=\sigma_{t 0}+c \eta \cos \varphi\left[\frac{d \psi}{d x}+m \frac{\psi}{x}\right] \text {. . . . . . . (4) }
\end{aligned}
$$

1) s. Füplls » Festigkeitslehre* Band III I gog S. 246 .

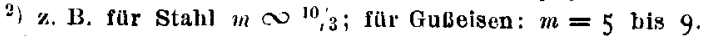


Setzen wir in diesen beiden Gleichungen als Sonderwerte für $\eta$ die Grenzwerte. $\left( \pm \begin{array}{l}h \\ 2\end{array}\right)$ ein, so erhalten wir die Spannungen $\sigma_{r}$ und $\sigma_{t}$ in den Aubenund Innenfasern der Platte.

Um nun für jeden Punkt der Platte die Werte $\sigma_{r}$ und $\sigma_{t}$ berechnen zu können, wollen wir vorerst für $\sigma_{r 0}$, $\sigma_{t 0}$ und $\psi$ Beziehungen aufstellen.

\section{4) Berechnung von $\sigma_{r o}$ unter Vermittlung der Gleichgewichtsbedingung der am Plattenelement angreifenden Kräfte.}

Wir denken uns gemäß Fig. 3 im Abstand $x$ von der Symmetrieachse aus der Platte ihrer ganzen Höhe nach ein Element in Richtung des Meridians und des Parallelkreises von vorerst unendlich kleinen Grundrilb-Abmessungen herausgeschnitten. Die vier Schnittflichen sollen alle senkrecht stehen zu den Meridianund Parallelkreis-Mitteliasern des Elementes. Zwei dieser Schnittflächen sollen Ebenen sein, durch die Symmetrieachse gehen und unter sich den Winkel $\boldsymbol{d} \alpha$ einschließell. Winkelrecht auf die von ihnen gebildeten Seitenflächen $G C D H$ und $E F K I$ des Plattenelementes wirken die Tangentialspannungen $\sigma_{\ell}$ im Abstand $\eta$ von der mittleren Neridianfaser und $\sigma_{t}$ in der Mitteliaser selbst, und diese haben die Richtung der Tangenten an die bezüglichen Parallelkreise. Die auf diese beiden Seitenflächen wirkenden Resultierenden seien $T$, welche ebenfalls den Winkel $\boldsymbol{d} \ll$ miteinander einschließen.

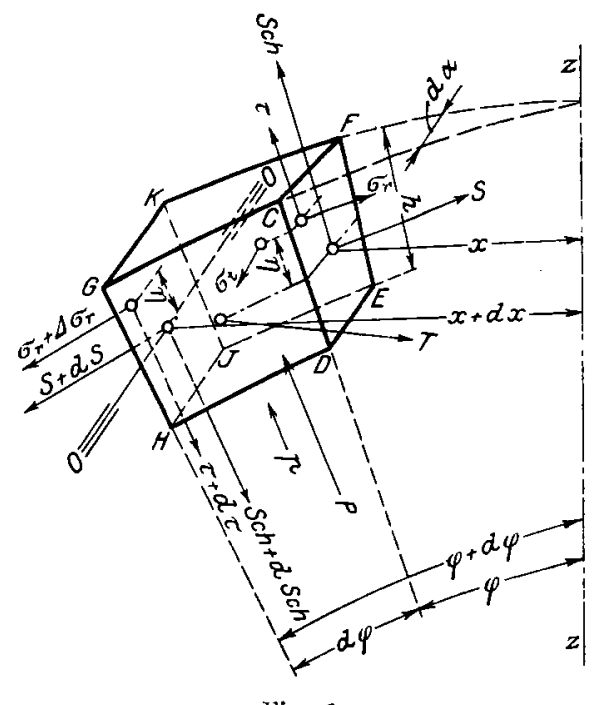

Fir. 3 .

Die beiden anderen Schnittflächen für das Plattenelement, nämlich $C D E F$ und $G H I K$, sind eigentlich Kegelflächen, dürfen ihrer Kleinheit wegen jedoch als Ebenen betrachtet werden. Sie schließen unter sich den Winkel $d q$ und mit der Symmetrieachse $z-z$ die Winkel $\varphi$ und $(\varphi+d \varphi)$ ein. Die in diesen Flächen herrschenden Normalspannungen seien $\sigma_{r}$ und $\left(\sigma_{r}+\boldsymbol{d} \sigma_{r}\right)$ im Abstand $\eta$ vom mittleren Parallelkreis sowie $\sigma_{r 0}$ und $\left(\sigma_{r 0}+\boldsymbol{d} \sigma_{r 0}\right)$ in den mittleren Parallelkreisen selbst. Sie ergeben die auf die ganzen Flïchen wirkenden Resultierenden $S$ und $(S+d S)$.

In den beiden zuletzt betrachteten Schnittflachen wirken aulber den Normalspannungen noch Schubspannungen $\tau$ und $(\tau+d \tau)$. welche die Resultierenden $S c h$ und $(S c h+d S c h)$ erzeugen. Es sei gleich an dieser Stelle hervorgehoben, daß diese in Richtung des Krümmungshalbmessers wirkenden Schubspannungen in der Mitte der Flächen einen Höchstwert $\tau_{0}$, an Rand der Flächen, z. B. an den Kanten $C F$ und $D E$ jedoch den Wert 0 haben. Ihr Mittelwert $\tau_{m}$ tritt also nicht in der wagerechten Mittellinie der Seitenfläche $C D E F$ auf, doch wollen wir dies nicht weiter verfolgen, da sich dieser Wert aus der Rechnung ganz eliminieren liaßt.

Weil die Seitenflïchen nicht Quadrate sondern trapezähnliche Flächen sind, so sind die in ibnen wirkenden mittleren Normalspannungen $\sigma_{r m}$ und $\sigma_{t m}$ auch nichṭ genau gleich den in dẹn Mittelfasern herrschenden Spannungen $\sigma_{r n}$ 
und $\sigma_{t h}$; doch ist der Unterschied so klein, daß man davon absehen darf, ohne einen unzulässigen Fehler zu begehen.

Die Größen der auf die Seitenflächen des Plattenelementes wirkenden Resultierenden ergeben sich als Produkte aus den Flächen und den in ihnen wirkenden mittleren Spannungen.

Wir können für sie folgende Aufstellung machen:

Fidche

$C D E F=\boldsymbol{E} d \boldsymbol{\alpha} h$

$C D E F=x d \alpha h$;

$C D H G=F E I K=d s h ;$

$D E I H=\left(x+\frac{d x}{2}\right) d \alpha d s ;$

$=\left(x+\frac{d x}{2}\right) \frac{d x}{\cos p} d \psi$ mittl. Spannung

$\sigma_{r 0}$ (normal);

$\tau_{m}$ (Schub);

$\sigma_{\text {ti }}$ (normal);

$p$

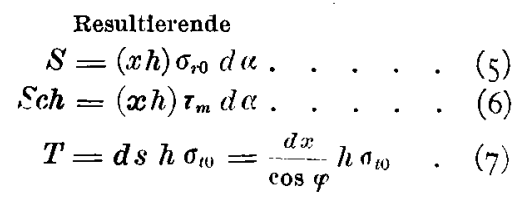

$$
P=p \cdot \frac{d x}{\cos p}\left(x+\frac{d x}{2}\right) d x .
$$

(Im Ausdruck für $P$ werden wir $\frac{d x}{2}$ gegenüber $x$ nicht vernachlässigen mit Rücksicht auf die später durchgeführte Rechnung mit endlich kleinen Differenzen statt unendlich kleinen Differentialen, weil sonst bei kleinem $x$ der Fehler zu groß würde.)

An Hand von Fig. 4, d. i. der Seitenansicht des Plattenelementes, kann man für dieses Element folgende Gleichgewichtsbedingung für die an ihm wirkenden Kräfte aufstellen: Wir vergleichen die in Richtung der Normalkraft $(\boldsymbol{S}+d \boldsymbol{S})$ fallenden Komponenten:

$$
S+d S=S \cos d q+S c h \sin d q+P \sin \frac{d r}{2}+2 T^{\prime} \cos (\varphi+d \eta)
$$

Berücksichtigt man wiederum, daß $d \varphi$ sehr klein, so daß $\cos d \boldsymbol{q} \sim$ I $\sin d \eta \propto d \tau, \cos (\eta+d \eta) \sim \cos \varphi$, so bleibt

$$
d S=S \operatorname{ch} d \varphi+P \frac{d \varphi}{2}+2 T^{\prime} \cos q
$$

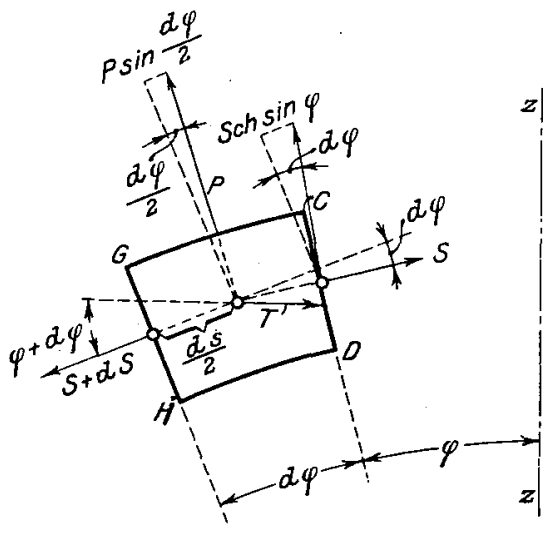

Fig. 4.

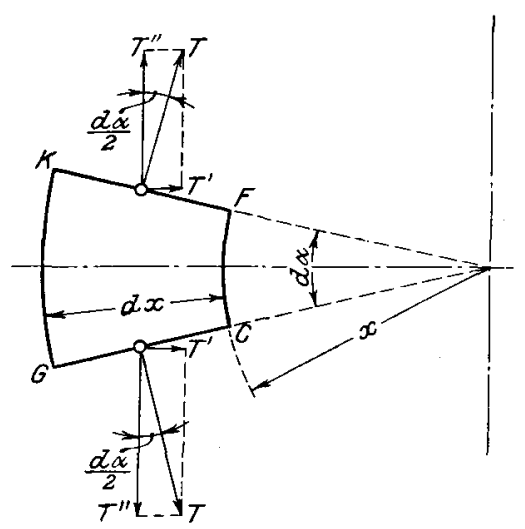

Fig. 5.

Hierin ist $T^{\prime}$ die in Richtung des Halbmessers $x$ fallende Komponente von $T$

Nach Gl. (5) ist

$$
T^{\prime}=T \sin \frac{d x}{2} \sim T \frac{d \alpha}{2} ; \text { (vergl. Fig. 5). }
$$

$$
\begin{aligned}
S & =(x h) \sigma_{r 0} d u, \\
d S & =\left[(x h) d \sigma_{r 0}+\sigma_{r 0} d(x h)\right] d \alpha .
\end{aligned}
$$


Unter Verwendung der Gl. (6) bis (8) erhält man nach Kürzung des Faktors $d x$ :

$$
(x h) d \sigma_{r 0}+\sigma_{10} d(x h)=\tau_{m}(x h) d \eta+p \frac{d x}{\cos \varphi}\left(x+\frac{d x}{2}\right) \frac{d \varphi}{2}+i \frac{d x}{\cos \varphi} \sigma_{t 0} \cos \varphi \quad \text {. (9). }
$$

Fig. 6 zeigt die Möglichkeit, die mittlere Schubspannung $\tau_{m}$ durch die Normalspannung $\sigma_{t 0}$ und die äußere Belastung $p$ auszudrücken und sie hierdurch aus der Rechnung zu eliminieren.

Um die Rechnung nach Möglichkeit zu verallgemeinern, wollen wir eine gewölbte Platte betrachten, welche in der Mitte eine gleichachsige Bohrung vom Halbmesser $\boldsymbol{x}_{\boldsymbol{i}}$ hat. Aus dieser Platte schneiden wir ein Ringteil mit dem äußeren Halbmesser $x$ und dem Zentriwinkel $d \alpha$ heraus. Dieser Ringausschnitt ist in Fig. 6 in der Seitenansicht dargestellt.

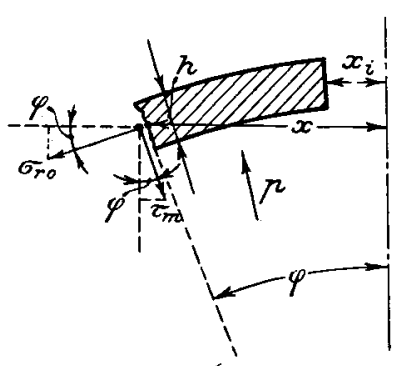

FIg. 6. Aus ihr lassen sich folgende Beziehungen ablesen:

$$
\begin{aligned}
& \left(x^{2}-x_{i}{ }^{2}\right) \pi\left(\frac{d \alpha}{2 \pi}\right) p=x d u h\left(\tau_{m} \cos \varphi+\sigma_{r i} \sin \varphi\right) \\
& \left.(x h) \tau_{m}=\frac{p}{2}\left(\frac{x^{2}-x_{i}{ }^{2}}{\cos \psi}\right)-(x h) \frac{\sin \varphi}{\cos \varphi} \sigma_{r 0} \text {. . . . . . . . . (г ( } 0\right) \text {. }
\end{aligned}
$$

Die rechte Seite dieser Gleichung werde in G1. (9) eingesetzt:

$$
\begin{aligned}
(x h) d \sigma_{r 0}+\sigma_{r 0} d(x h)=\frac{p}{2} \cdot\left(x^{2}-x_{i}^{2}\right) \frac{d x}{\rho \cos ^{2} \varphi} & -(x h) \sigma_{r 0} \sin \varphi \frac{d x}{\rho \cos ^{3} \varphi} \\
& +\frac{p}{2} \frac{d x^{2}}{\rho \cos ^{2} \varphi}\left(x+\frac{d x}{2}\right)+h d x \sigma_{t 0} .
\end{aligned}
$$

Hieraus finden wir:

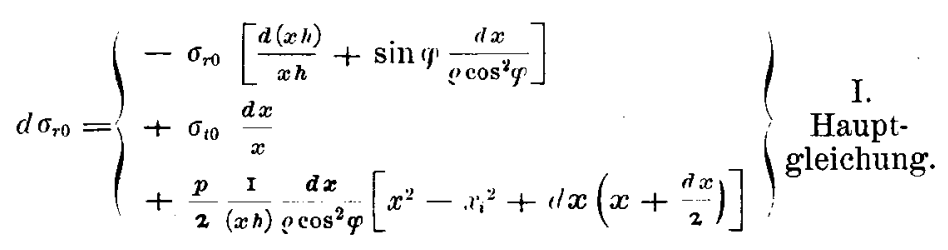

Diese I. Hauptgleichung hat die Form:

$$
\left.d \sigma_{r 0}=-\sigma_{r 0}\left(1_{5}\right)+\sigma_{10}(\mathrm{I} 6)+(24) . . . \quad . \quad . \quad \text { ( } \mathbf{I} \mathbf{a}\right),
$$

wo die Ziffern in () Zahlenwerte bedeuten, die abhängig sind von der Form und der äußeren Belastung der Platte und der Lage des augenblicklich zu untersuchenden Punktes $\boldsymbol{A}$ auf der Mittelfaser des Meridianschnittes.

Würde man für den Halbmesser $x$ die mittlere Radialspannung $\sigma_{r 0 x}$ kennen, so lieferte die Hauptgleichung (I) den Wert fïr die mittlere Radialspannung $\sigma_{r 0}(x+d x)$ im Halbmesser $(x+d x)$

$$
\sigma_{r 0}(x+d x)=\sigma_{r j x}+\left.d \sigma_{r 0}\right|_{x} ^{x+d x} \text {. . . . . . . (II). }
$$

\section{5) Berechnung von $\sigma_{t 0}$, hergeleitet aus der Dehnung der Platte.}

Der Parallelkreis mit dem Halbmesser $x$, der die gestreckte Länge $(2 \pi x)$ hat, dehnt sich um das Stück $J(2 \pi x)$, wenn in Richtung der Tangente die spezifische Spannung $\sigma_{t 0}$, senkrecht dazu die Spannung $\sigma_{r 0}$ wirkt, und zwar ist;

$$
\Delta(2 \pi x)=\frac{2 \pi x}{E}\left(r_{t},-\frac{\sigma_{r_{0}}}{m}\right) ;
$$


danach

$$
\Delta x=\frac{x}{E}\left(\sigma_{t 0}-\frac{\sigma_{r 0}}{m}\right) .
$$

Die Differenzierung dieser Gleichung liefert die Dehnung des Halbmesserelementes $(d \boldsymbol{x})$

$$
\left.\boldsymbol{\Delta}(d \boldsymbol{x})=\frac{d x}{\boldsymbol{E}}\left(\sigma_{i 0}-\frac{\sigma_{r 0}}{m}\right)+\frac{x}{\boldsymbol{E}}\left(d \sigma_{\iota_{0}}-\frac{d \sigma_{r 0}}{m}\right) . . \text {. . (I } 2\right) .
$$

Für diese Dehnung können wir noch einen zweiten Ausdruck aulistellen:

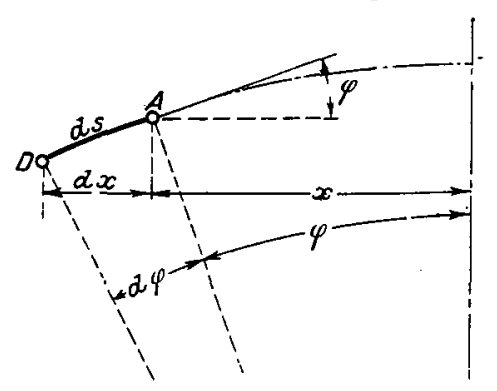

Fig. 7.

Wir denken uns gemäß Fig. 7 aus der mittleren Meridianfaser im Abstand $x$ von der Symmetrieachse bei $A$ ein Element von der Lünge $A D=d s$ herausgegriffen.

$$
\text { Weil }
$$

$$
d x=d s \cos \varphi,
$$

so ist auch die durch die Belastung eriolgte Aenderung von $d x$, das ist:

$$
\begin{aligned}
J(d x) & =\Delta(d s \cos \varphi) \\
& =\Delta(d s) \cos q+d s \Delta(\cos q)
\end{aligned}
$$

Nun ist

$$
\begin{aligned}
& \left.\Delta(d s)={ }_{E}^{d s}\left(\sigma_{r 0}-\frac{\sigma_{t_{0}}}{m}\right) . . . . . \quad . \quad \text { (I } 3\right), \\
& \Delta(\cos \eta)=-\sin \varphi \Delta \varphi=-\sin \eta \cdot \psi . . \quad . \quad . \quad \text { (I } 4) \text {, }
\end{aligned}
$$

demnach

$$
\begin{aligned}
& \Delta(d x)={ }_{E}^{d s}\left(\sigma_{r 0}-\sigma_{t 0}\right) \cos q-d s \sin \phi \cdot \psi \\
& \left.\Delta(d x)={ }_{E}^{d x}\left(\sigma_{r 0}-\frac{\sigma_{t 0}}{m}\right)-d x \operatorname{tg} \gamma \cdot \psi \cdot . \quad . \quad . \quad . \quad \text { ( } 15\right)
\end{aligned}
$$

Die Aenderung von $(d x)$ ist das Ergebnis zweier Formänderungen, nämlich der Läingenänderung und der Richtungsänderung des Meridian-Liementes $d s$.

Durch Gleichsetzen der rechten Seiten der Gl. (I2) und (I5) erhalten wir

$$
{ }_{E}^{d x}\left(\sigma_{t 0}-\frac{\sigma_{r 0}}{m}\right)+\frac{2}{E}\left(d \sigma_{t 0}-\frac{d \sigma_{r 0}}{m}\right)=\frac{d x}{E}\left(\sigma_{r 0}-\frac{\sigma_{t 0}}{m}\right)-d x \psi \operatorname{tg} \varphi
$$

Ls werden beide Seiten dieser Gleichung mit $\frac{p}{a}$ nultipliziert und die Klammern aufgelöst:

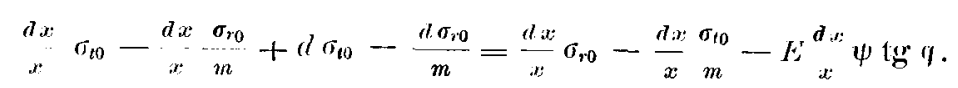

Daraus finden wir

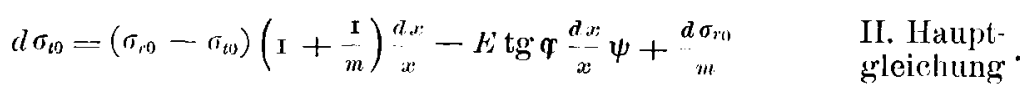

Diese Gleichung hat die Form:

$$
d \sigma_{t 0}=\left(\sigma_{t 0}-\sigma_{t 0}\right)(\mathrm{I} 7)-\boldsymbol{\psi}(27)+\frac{d \sigma_{r 0}}{m} \quad . \quad . \quad .
$$

Für die nur durch Fliehkräfte beanspruchte umlaufende scheibe fand ich in meiner diesbezüglichen, eingangs erwähnten Arbeit die Ausdrücke (in die hịer gewählte Bezeichnungsweise übersetzt):

$$
\begin{aligned}
& d \sigma_{r 0}=-\sigma_{r 0}(\ldots)+\sigma_{t 0}(\ldots)+(\ldots), \\
& d \sigma_{t 0}=\left(\sigma_{r 0}-\sigma_{t 0}\right)\left(\mathrm{I}+\frac{\mathrm{r}}{m}\right) \underset{p}{d x}+\frac{d \sigma_{r 0}}{m}
\end{aligned}
$$


Der Aufbau der Formel fïr $d \sigma_{r 0}$ ist genau der gleiche wie derjenige del hier gefundenen Formel (I) für die Platte, nur dals natürlich für die (....) Ausdrücke andere Werte in Frage kommen. Zu der Gleichung für $d \sigma_{t 0}$ kommt laut Gl. (IIa) für die Platte gegenüber derjenigen für die umlanfende Scheibe nur noch der Summand $-\psi(27)$ hinzu. Es ist also möglich, beide Rechnungen niteinander zu vereinigen, wodurch das Mittel an die Hand gegeben wird, eine umlaufende Scheibe zu berechnen, die außer durch Fliehkräfte auch noch durch einen einseitig wirkenden, gleichmäßig verteilten Druck $p$ belastet wird. Derartige Falle kommen vor in Reaktions-, seltener auch in Aktionsdampiturbinen.

Unter der vorläufig noch unzutreffenden Annahme, man kenne von der Platte für den Halbmesser $\boldsymbol{x}$ die mittlere Tangentialspannung $\sigma_{t 0 x}$, liefert Hauptgleichung (II) den Wert für die mittlere Tangentialspannung im Halbmesser $(x+d x)$, indem man die Gleichung autstellt:

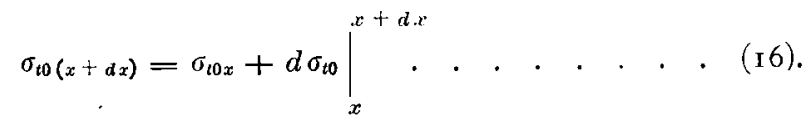

\section{6) Berechnung von $\frac{d \psi}{d x}$ und $\psi$ unter Benutzung der Momenten- gleichung.}

Wir stellen zu diesem Zwecke für das in Fig. 3 axonometrisch dargestellte Körperelement $C D E F G H I K$ die Momentengleichung auf. Als Momentenachse greif́en wir die Achse $o-o$ heraus, welche im Alsstand $(x+d x)$ von der Symmetrieachse $z-z$ und senkrecht zu ihrer Richtung mitten durch die äußere Bogrenzungsfläche $G H I K$ des Plattenelementes läuft. Bei Gleichgewicht muß dic Summe der Momente aller äußeren Kräfte, bezogen auf die Achse o-o, gleich null sein.

An dem Ṕlattenelement wirken folgende äußere Krräfte auf Verdrehung un die Achse $0-o$ :

a) auf die der Symmetrieachse zugekehrte Begrenzungsfläche CDFF:

Die Normalspannungen, deren Wirkung ersetzt werden kann:

I) durch eine im Mittelpunkt der Fläche angreifende Einzelkraft $S=x$ d $\boldsymbol{h} \sigma_{r 0}$ wirkend am Hebelarm $d s \sin (d \boldsymbol{q})$.

2) ein Moment $" \boldsymbol{M}_{\jmath^{\prime}}$, auf welches später einzugehen ist.

3) die Schubkraft $S c h=x d \alpha h \tau_{m}$ am Hebelarm $d s \cos (d \varphi)$.

b) Auf die beiden Seitenflächen $G C D H$ und $E F K I$ wirken Normalspannungen, deren Einfluß ersetzt werden kann:

I) durch die Normalkraft $T \sim d s h \sigma_{10}$.

Von ihr kommt als drehend um die Achse o-o nur die Komponente $T^{\prime}=T \sin \frac{d a}{2} \sim T \frac{d a}{2}$ in Betracht (vergl. Fig. 5). Die beiden anderen Komponenten $T^{\prime \prime}=T \cos \frac{d \alpha}{2}$ verlaufen parallel zur Achse $o-o$ und ergeben daher kein Drehmoment (vergl. Fig. 5 und Fig. 3). Die beiden Komponenten $T^{\prime}$ wirken je an Hebelarm ${ }_{2}^{d s} \sin (q+d q) \propto \frac{d s}{2} \sin \varphi$ (siehe Fig. 4).

2) durch das Yoment " $\boldsymbol{M}_{\boldsymbol{s}_{t}}$ (ler Spannungen $\sigma_{t}$, auf welches wir später zurückkommen.

c) Normal zur unteren Begrenzungsfläche $H D K I$ und in die Mitte derselben konzentriert gedacht, wirkt die Kraft $P=\left(x+\frac{d x}{2}\right) d \alpha d s p$ ạm Hebelarm $\begin{gathered}d s \\ 2\end{gathered}$. 
[Auch hier wollen wir mit Rücksicht auf die spätere Rechnung mit kleinen Differenzen statt Differentialen von einer Vernachlässigung des Wertes $\frac{d x}{2}$ absehen.]

Alle die unter $a, b$ und $\mathrm{c}$ genannten Momente versuchen, das Plattenelement im einen oder anderen Sinn um die Achse o-o, Fig. 3, zu drehen. Es kann in seiner Lage, d. h. im Gleichgewicht, nur dadurch gehalten werden, daß auf die äußere Begrenzungsfläche $G H I K$ das bisher noch nicht berücksichtigte Moment $M_{\sigma_{r}}+\Delta \sigma_{r}$ wirkt, welches gleich ist der algebraischen Summe jener vorgenannten Spannungsmomente und entgegengesetztes Vorzeichen hierzu hat Wir wollen jene Momente zuerst nach der Größe, sodann nach dem Vorzeichen bestimmen:

$\mathrm{Zu}$ a I)

$$
M_{S}=S d s \sin (d q) \infty S \frac{d x}{\cos \varphi} d \varphi
$$

Nun ist

$\mathrm{Zu}$ a 3)

$$
\begin{aligned}
& d \Phi=\frac{d s}{\rho}=\frac{d x}{\rho \cos \varphi} .
\end{aligned}
$$

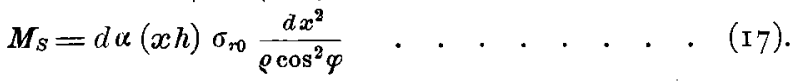

Aus Gl. (Io) finden wir für $S c h=(x h) \tau_{m} d r$

$$
\begin{gathered}
S c h=d l e\left[\frac{p}{2}\left(\begin{array}{c}
x^{2}-x_{i}{ }^{2} \\
\cos \varphi
\end{array}\right)-(x h) \sigma_{r o} \operatorname{tg} \Upsilon\right] . \\
\left.M_{S c h}=d \ll \frac{p}{2}\left(x^{2}-x_{i}^{2}\right) \frac{d x}{\cos ^{2} \varphi}-d \propto(x h) \sigma_{r o} \sin \varphi \frac{d x}{\cos ^{2} \varphi} \text {. . . (I } 8\right) .
\end{gathered}
$$

$\mathrm{Zu} \quad a_{2}$ ) In Fig. 8 ist das Plattenelement in gleicher Weise dargestellt, wie in Fig. 3, und es sind an dem einen Rand der inneren Begrenzungsfläche $C D E F$

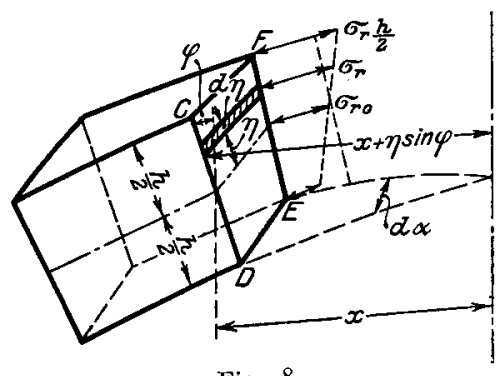

Fig. 8 . in einem gewissen Maßstab die Spannungen $\sigma_{r}$ der Größe und Richtung nach aufgetragen. In der Mitte der Kante $E F$ herrscht die Spannung $\sigma_{r 0}$, im Abstand $\eta$ von der Mitte die Spannung $\sigma_{r}$. Sie darf iiber dem gestrichelten Flächenstreifen von der Höhe $d \eta$ und der angenäherten Breite $x d \alpha$ als unveränderlich angenommen werden. Das Moment der Spannungsdifferenzen $\left(\sigma_{r}-\sigma_{r 0}\right)$, welches auf die Begrenzungsfläche $C D E F^{\prime}$ wirkt, und welches wir abkürzungsweise mit $M_{\sigma_{r}}$ bezeichnen wollen, ist gleich der Summe aller Produkte, die aus der Multiplikation nachstehender drei Faktoren entstehen:

I) dem in Fig. 8 gestrichelten Flächenelement $d t$,

2) dem Unterschied der in diesem Flächenelement $d f$ herrschenden Spannung $\sigma_{r}$ gegenüber der in der Mittelfaser herrschenden Spannung $\sigma_{r 0}$, also der Differenz $\left(\tau_{r}-\sigma_{r 0}\right)$,

3) dem Abstand $\eta$ des Flächenelementes von der Mittelfaser.

Hierin ist

$$
M_{\sigma_{r}}=\int_{-\frac{h}{2}}^{+\frac{h}{2}} d f\left(\sigma_{r}-\sigma_{r 0}\right) \eta .
$$

$$
d f=(x+\eta \sin \varphi) d \alpha d \eta
$$


Für $\left(\sigma_{r}-\sigma_{r_{0}}\right)$ wollen wir an Hand von Gl. (3) einsetzen

$$
\left(\sigma_{r}-\sigma_{r 0}\right)=c \eta_{\eta} \cos \varphi u \text {, }
$$

wo

$$
\begin{aligned}
& u \equiv\left[m \frac{d \psi}{d x}+\frac{w}{x}\right] \text {. . . . . . . . . ( (19), } \\
& +\frac{h}{2} \\
& M_{\sigma_{r}}=\int_{-\frac{h}{2}} c \eta \cos \eta u(x+\eta \sin \eta) d \alpha d \eta \eta .
\end{aligned}
$$

In diesem Integral ist nur $\eta$ als Veränderliche, alle übrigen Größen sind als Konstante $\mathrm{zu}$ betrachten.

$$
\begin{aligned}
& M_{\sigma_{r}}=d u c \cos q u\left[x \int \eta^{2} d \eta+\sin \eta \int \eta^{3} d \eta\right] ; \quad \int_{-h}^{+\frac{h}{2}} \eta^{2} d \eta=\frac{h^{3}}{12} ; \quad \int_{-\frac{h}{2}}^{+\frac{h}{2}} \eta^{3} d \eta=0 . \\
& M_{\sigma_{r}}=d \varkappa c \cos \tau u x \frac{h^{3}}{12} .
\end{aligned}
$$

Setzen wir den Wert für $u$ wieder ein aus Gl. (I9), so erhalten wir:

$$
M_{\sigma_{r}}=d x c x \frac{h^{3}}{\mathrm{I} 2} \cos \varphi\left[m_{d x}^{d \psi}+\frac{\psi}{x}\right] \text {. . . . . . (20). }
$$

Wir werden später sehen, daß wir noch des Wertes $d M_{\sigma_{r}}$ bedürfen, d. h. des Betrages, um den sich $\boldsymbol{M}_{\boldsymbol{\sigma}_{r}}$ ändert, wenn wir von $x$ um $d \boldsymbol{x}$ vorwärts gehen. Wir erhalten, indem wir die Gl. (20) nach $x$ differenzieren:

$$
d \boldsymbol{M}_{\sigma_{r}}=d \kappa_{\mathrm{I} 2}^{c}\left\{\begin{array}{l}
\left(m x h^{3} \cos \boldsymbol{\eta}\right) \frac{d^{3} \psi}{d x^{2}} d x+d\left(m x h^{3} \cos \eta\right) \frac{d \psi}{d x} \\
+h^{3} \cos \varphi d \psi+d\left(h^{3} \cos \varphi\right) \psi
\end{array}\right\} \text { (2I). }
$$

$\mathrm{Zu} \mathrm{b} \mathrm{I)}$

$$
\Sigma M_{T}=2 T^{\prime} \frac{d s}{2} \sin \gamma=2 T \sin \frac{d r}{2} \frac{d s}{2} \sin \gamma .
$$

Statt $\Sigma M_{T}$ wollen wir einfach setzen $I_{T}$.

$$
\begin{gathered}
M_{T}=d \alpha(d s h) \sigma_{t 0} \frac{d s}{2} \sin \eta \\
M_{T}=d \varkappa\left(\frac{h}{2} \frac{d x^{2}}{\cos ^{2} \varphi} \sin \gamma\right) \sigma_{t 0}
\end{gathered}
$$

$\mathrm{Zu} \mathrm{b} \mathrm{2)} \mathrm{Das} \mathrm{Moment} \boldsymbol{M}_{\sigma_{t}}$ der Spannungen $\sigma_{t}$, oder was das Gleiche besagt,

- der Spannungsdifferenzen $\left(\sigma_{t}-\sigma_{t 0}\right)$, welches auf jede der beiden Seitenflächen $G H D C$ und $K I E F$ von Fig. 3 wirkt, ist gleich der Summe aller Produkte, die aus der Multiplikation nachstehender drei Faktoren entstehen: (vergl. Fig. 9).

I) dem Flächenelement $d f$ von der Höhe $d \eta$, welches im Abstand $\eta$ von der Mittel-Meridianfaser zur Kante $G C$ parallel verläuft.

2) von dem Unterschied der in diesem Flächenelement herrschenden Spannung $\sigma_{t}$ gegenüber der Spannung $\sigma_{t 0}$ in der Mittelfaser die in die Richtung senk-

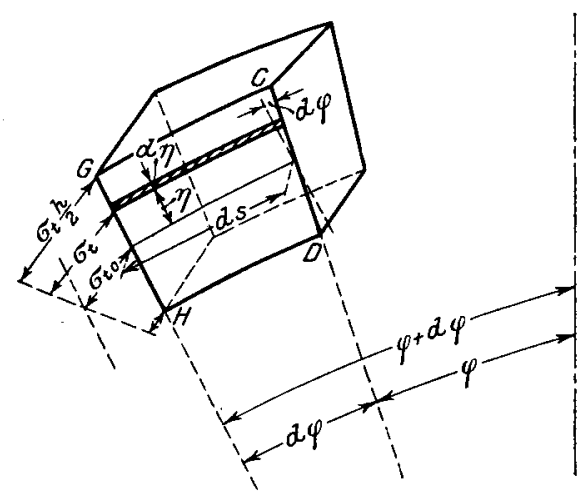

Fig. 9. 
recht zur' Symmetricachse entiallende Komponente, also

$$
\left(\sigma_{t}-\sigma_{t 0}\right) \sin \frac{d \alpha}{2} \sim\left(\sigma_{t}-\sigma_{t 0}\right) \frac{d \alpha}{2} .
$$

3) dem Abstand $\eta$ des Flächenelementes von der Mittelfaser, multipliziert mit $\cos (p$

$$
M_{\sigma_{t}}=2 \int_{-\frac{h}{2}}^{+\frac{h}{2}} d f\left(\sigma_{t}-\sigma_{t 0}\right){ }_{2}^{d a} \cdot \eta \cos \eta
$$

Hierin ist nach Fig. 9

Gl. (4) besagt:

$$
\begin{gathered}
d f=(d s+\eta d \boldsymbol{q}) d \eta . \\
\left(\sigma_{t}-\sigma_{t 0}\right)=c \eta \cos \boldsymbol{\varphi}\left[\frac{d \psi}{d x}+m \frac{\psi}{x}\right] .
\end{gathered}
$$

Setzen wir vorïbergehend als Abkürzung

so ist

$$
v=-\left[\frac{d \psi}{d x}+m \frac{\psi}{x}\right] \quad . \quad . \quad . \quad . \quad . \quad . \quad . \quad . \quad(23),
$$

$$
H_{\boldsymbol{v}_{t}}=2 \int_{-{ }_{-}}^{+\frac{h}{2}}(d s+\eta d \varphi) d \eta c \eta \cos ^{2} \boldsymbol{\eta} \frac{d \alpha}{2} \eta .
$$

Auch hier, wie bei der Ausrechnung von $M_{\sigma_{r}}$ sind bei der Integration alle Gröfien auber $y$ als Konstante zu betrachten.

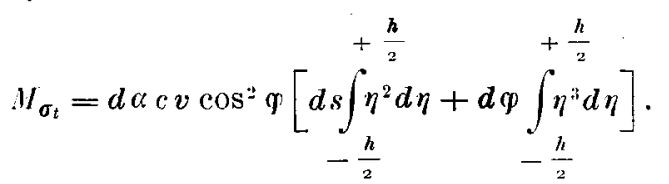

Durch analogen Rechnungsgang wie für $M_{\sigma_{r}}$ finden wir:

\%u c)

$$
M_{\sigma_{t}}=d \mu c d x \underset{\mathrm{I} 2}{h^{3}}\left[\frac{d \psi}{d x}+m \frac{\psi}{x}\right] \cos \varphi \quad \text {. . . . . (24). }
$$

$$
\begin{aligned}
& M_{P}=P \frac{d s}{2}=d s\left(x+\frac{d x}{2}\right) d \alpha p \frac{d s}{2} \\
& \quad M_{P}=d \alpha \frac{p}{2}\left(x+\frac{d x}{2}\right) \frac{d x^{2}}{\cos ^{2} \varphi} \quad \cdot \quad \cdot \quad . \quad . \quad . \quad . \quad . \quad(25) .
\end{aligned}
$$

Nunmehr sind von diesen Momenten noch die Vorzeichen zu bestimmen. Sie erhalten bei der Summierung das positive oder negative Vor'zeichen, ic nachdem sie verstärkend oder verschwächend auf die Plattenkrümmung in dem Querschnitt hinwirken, der die. Drehachse o-o, Fig. 3, enthält. Es wirken auf diese Krümmung:

$M_{S}$ verstärkend, also Vorzeichen + ,

$\boldsymbol{M}_{\text {Sch }}$ verschwächend, also Vorzeichen -,

$M_{\sigma_{r}}$ verstärkend, wenn die Spannungsunterschiede $\left(\sigma_{r}-\sigma_{r v}\right)$ über der

Mitte des Querschnittes positiv sind, somit dann $M_{\sigma_{r}}+$

$M_{T}$ verstärkend, also +,

$M_{\sigma_{t}}$ analog $M_{\sigma_{r}}$, also + ,

$M_{P}$ verschwächend, also -

$$
M_{\left\langle\sigma_{r}+d \sigma_{r}\right\}}=M_{\sigma_{r}}+M_{S}-M_{\text {Sck }}+M_{7}+M_{\sigma_{t}}-M_{P_{,}}
$$


Num kann auch geschrieben werden:

$$
M_{\left(\sigma_{r}+{ }^{\prime} \sigma_{r}\right)}=M_{\sigma_{r}}+d M_{\sigma_{r}}
$$

und daraus ergibt sich

$$
d M_{\sigma_{r}}=M_{S}-M_{S c h}+M_{T}+M_{\sigma_{t}}-M_{P} \ldots \text {. . . . (26). }
$$

Hierin setzen wir die Werte ein aus den

$$
\text { Gl. (2I), (17), (18), (22), (24), (25). }
$$

Alle diese Werte enthalten $d \alpha$, welchen Wert wir besonders vorangestellt haben, als Hinweis darauf, daß man damit kiirzen kann. Fs bleibt:

$$
\begin{aligned}
& \text { I } 2\left[\left(m x h^{3} \cos \varphi\right) \frac{d^{2} \psi}{d x^{2}} d x+d\left(m x h^{3} \cos \varphi\right){ }_{d x}^{\lambda \psi}+h^{\prime \prime} \cos \varphi d \psi+d\left(h^{\prime \prime} \cos \varphi\right) \psi\right. \text { aus (2I) }
\end{aligned}
$$

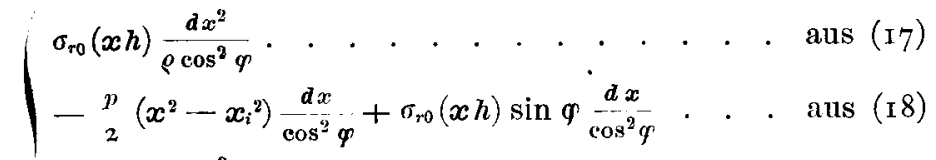

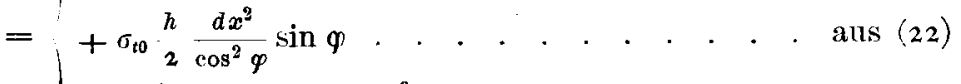

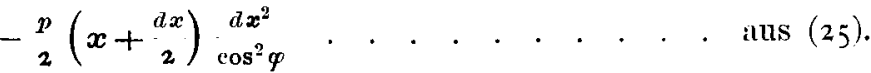

Die Multiplikation dieser Gleichung mit $\frac{12}{c d x}$ gibt

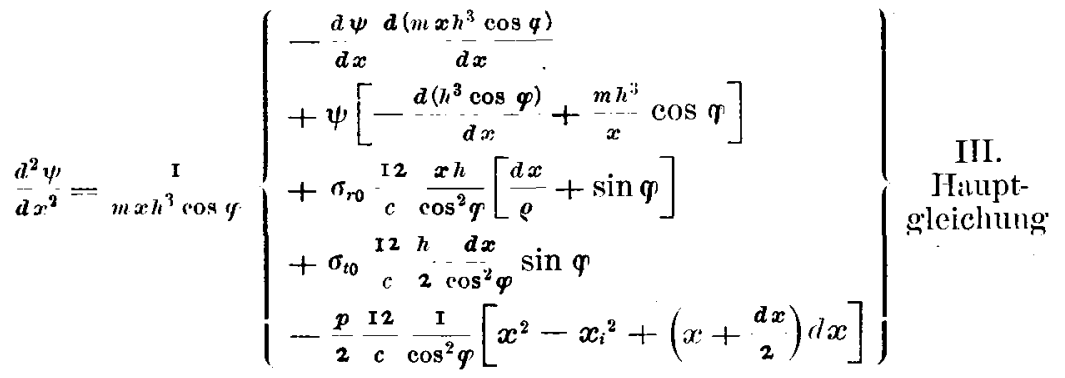

Dicse Hauptgleichıng hat die schematische Form:

$$
d^{d^{2} \psi}=\underset{(40)}{\text { I }}\left\{(32) \sigma_{r 0}+(36) \sigma_{t 0}-(42) \frac{d \psi}{d x}+(49) \psi-(5 \mathrm{I})\right\} . \quad \text { (III a), }
$$

wo die in den runden Klammern () stehenden Ziffern Faktoren bedeuten, welche lediglich abhängig sind von der Form und der äuleren Belastung $\boldsymbol{p}$ der Platte, nicht aber z. B. von der Randbedingung, d. h. daron, ob die Platte am Rand eingespannt sei oder frei aufliege. Ob die Platte in der Mitte eine Bohrung hat oder nicht, kommt lediglich im Summand (5I) zum Ausdruck, indem dort der Summand $x_{i}^{2}$ einen von null verschiedenen Wert erhält oder nicht. Da ferner die Platte nicht eben zu sein braucht, sondern in jedem Punkt der Meridianmittelfaser eine andere Krümmung mit dem veränderlichen Halbmesser $\varrho$ und außerdem eine von P'unkt zu Punkt etwas verïnderliche D:cke $h$ haben kann, so ist dieses Rechnungsverfahren anwendbar auf gewölbte wie ebene $(\varrho=\infty$, $q=0$ ) Platten, volle und in der Mitte gelochte, am Außenrand frei aufliegende oder eingespannte Platten von verinderlicher Dicke.

Nach Ausrechnung der Gl. (III) erhalten wir: 


$$
\begin{aligned}
& -16- \\
& \left.\frac{d \psi^{\prime}}{d x}\right|_{x+d x}=\left.\frac{d \psi}{d x}\right|_{x}+\frac{d^{2} \psi}{d x^{2}} d x . \quad . \quad . \quad . \quad . \quad . \quad \text { (27) } \\
& \psi_{x+d x}=\psi_{x}+\left.\frac{d \psi}{d x}\right|_{x} d x \text {. . . . . . . . (28). }
\end{aligned}
$$

Hierbei erinnern wir uns stets, daß wir bei der Ausrechnung statt des unendlich kleinen Differentials eine zwar kleine, aber endliche Differenz $d x=x_{2}-x_{1}$ einzusetzen haben.

\section{- Gang der Rechnung.}

Für irgend einen Halbmesser $r_{2}$ der Meridian-Mittelfaser können mit Hülfe der drei Hauptgleichungen (I). (II) und (III) und der Nebengleichungen (11), (I6) und (27), (28) die Werte $\sigma_{r 0}, \sigma_{t 0}$ und $\psi$ berechnet werden, wenn die korrespondierenden Werte für den um das endliche, aber kleine Stück (dx) verschiedenen Halbmesser $x_{\mathrm{I}}$ bekannt sind.

Um die Werte der Normalspannungen und der Winkeländerungen für alle Punkte der Mittelfaser zu erhalten, nehmen wir für irgend einen Punkt dieser Faser Werte an und rechnen für alle aufeinanderiolgenden Werte von (dx) nach den Gl. (I), (II), (III) die Aenderungen der Normalspannungen und der Winkeländerung und sodann die Spannungen und Winkeländerung selbst aus. Am besten beginnt man innen an der Platte.

Stimmt nach der erstmaligen Durchrechnung das Endergebnis nicht mit den Randbedingungen, so ist die Rechnung von innen bis außen zu wiederholen. Während man aber bei der oben erwähnten Berechnung der umlaufenden Scheibe beim Beginn einer Durchrechnung nur mit einer Unbekannten $\left(\sigma_{t}\right) \mathrm{zu}$ variieren brauchte, sind es hier deren zwei, wie wir noch sehen werden.

\section{Regeln für den Beginn der Ausrechnung.}

I. Fall: Die Platte hat in der Mitte keine Bohrung. $x_{i}=0$.

Für irgend einen auf der Symmetrieachse im Abstand $\eta$ von der Mittelfaser entfernten Punkt ist

$$
\sigma_{r \gamma_{i} i}=\sigma_{t \gamma_{i} i} .
$$

Für den Schnittpunkt der Symmetrieachse mit der Mittelfaser des Meridianquerschnittes ist im besonderen

$$
\text { (fuir } x=0) \quad \sigma_{r 0 i}=\sigma_{i 0 i} \text {; }
$$

Es sei aber ganz besonders erwähnt, daß wegen der Durchbiegung der Platte

$$
\sigma_{r n t}=-1=\sigma_{r \tau_{i} \cdot}
$$

In einer besonderen Zahlentafel, wir wollen sie »Haupttafel « nennen, stellen wir alle in den für die ganze Durchrechnung erforderlichen Gleichungen vorkommenden Faktoren zusammen, welche abhängen von der Form und der vorgeschriebenen Belastung der Platte, nicht aber von den für die einzelnen Durchrechnungen zu machenden Annahmen. In welcher Weise dies geschieht, werden wir später an Hand eines Zahlenbeispieles zeigen. Wir wollen also voraussetzen, daß wir die in den Hauptgleichungen (Ia), (IIa) und (IIIa) durch die in ( ) stehenden Ziffern dargestellten festen Faktoren von vornherein festgestellt haben. 
Nun wählen wir einen ersten Wert für $\sigma_{r 0 i}=\sigma_{t 0 i}$; es sei dies $\sigma_{r 0 i}^{\prime}=\sigma_{i 0 i}^{\prime}$ und erhalten für ein erstes Intervall von der Größe $d x$ nach Gl. (Ia) den Wert $d \sigma_{r o}$ und sodann nach Gl. (11)

$$
\sigma_{, 0} \text { fiür } x=0+d x \text {. }
$$

Um mit der ersten Wahl $\sigma_{r 0 i}^{\prime}=\sigma_{t 0 i}^{\prime}$ nicht gar $\mathrm{zu}$ weit neben das Ziel zu schießen, machen wir folgende Ueberlegung: Der in Fig. I dargestellte Meridianschnitt (Plattenquerschnitt) erleidet eine mittlere Tangentialspannung $\sigma_{i}^{*}$, die man erhält, indem man die Projektion der Plattenkuppe auf die Meridianebene, d. i. die Flä.che $A_{1} J A_{2} J_{1} A_{1}$ mit der spezifischen äußeren Belastung $p$ multipliziert und dieses Produkt durch den Flächeninhalt des von $A_{1}$ über $J$ bis $A_{2}$ gemessenen Plattenquerschnittes $=\Sigma(d s h)$ dividiert.

$$
\begin{aligned}
& \sigma_{t}^{*}=\frac{\left(\text { Flăche } A_{1} J A_{2} J_{1} A_{1}\right) p}{\Sigma(d 8 h)} \\
& \sigma_{10 t}^{\prime} \text { ist zu wählen }=k \sigma_{t}^{*},
\end{aligned}
$$

wo $k$ in der Regel größer als I.

Für die Anwendung der Hauptgleichung (II a) kennen wir für das erste Intervall bereits $d \sigma_{20}$. Die übrigen Summanden in Gl. (IIa) sind für dieses erste Intervall $=0$, weil

$$
\begin{array}{rlrlrl}
\text { für } x & =0 & & \left(\sigma_{r 0}-\sigma_{t 0}\right) & =0 \\
\text { Es ist also } & \gg x & =0 & & =0 . \\
\psi & \gg x & =0 & d \sigma_{t 0} & =\frac{l \sigma_{r n}}{m} .
\end{array}
$$

Hiernach wird Gl. (16) angewendet.

Um nun mittels der Hauptgleichung (III a) füir das erste Interval $d \boldsymbol{x}$ den Ausdruck $\frac{d^{2} \psi}{d x^{2}}$ berechnen zu können, ist vorerst eine zweite Schätzung erforderlich, und zwar für $\left(\frac{d \psi}{d x}\right)_{x=0}$, nachdem wir ja vorher bereits $\sigma_{t o i}^{\prime}$ abschätzen mußten. Es fällt aber insbesondere dem in derartigen Rechnungen noch Ungeübten schwer, solche Winkelfunktionen auch nur einigermaßen annähernd abzuschätzen. Man hat eher Gefühl für die Wahl spezifischer Spannungen. Es empfiehlt sich daher, für $x=0$ nicht $\frac{d \psi}{d x}$, sondern den äquivalenten Wert $\sigma_{\frac{t}{2}} h_{i}$, d. h. die Spannung in der Begrenzungsfaser, also im Abstand $\eta=\frac{h}{2}$ von der Mittelfaser abzuschätzen. Sie ist natürlich im allgemeinen von $\sigma_{t 0 i}$ verschieden. Nun ist nach Gl. (4):

$$
\sigma_{t}=\sigma_{t 0}+c r \cos \varphi\left[\frac{d \psi}{d x}+m \frac{v}{x}\right]
$$

für $x=0$ ist $\cos \varphi=\mathrm{I}$; für $y=\frac{h}{2}$ ist $\sigma_{t}=\sigma_{t} \frac{h}{2}$.

$$
\text { " } x=0 \text { ० } \psi=0 \text {. }
$$

Damit ist aber noch nichts gesagt über das Verhältnis $\frac{\psi}{x}=\frac{0}{0}$. Wir finden jedoch für diesen Quotienten einen Anhalt, wenn wir bedenken, daß

$$
\text { fïr } \begin{aligned}
& x=d x \ldots \psi \psi=d \psi ; \\
& » \quad x=d x \ldots{ }^{\psi}=\frac{d \psi}{d x} .
\end{aligned}
$$

Von $x=d x$ bis zurück auf $x=0$ ändert sich der Ausdruck $\frac{\psi}{x}$ unendlich wenig, so daß wir auch für $x=0$ setzen dürfen $\frac{\psi}{x}=\frac{d \psi}{d x}$. 
Es ist also riul $x=0$ und $y=-\frac{h}{2}$

$$
\sigma_{t} h=\sigma_{10}+c_{2}^{h}\left[\begin{array}{l}
d \psi \\
d \boldsymbol{x}
\end{array}+m \frac{d \psi}{d \cdot \boldsymbol{x}}\right]
$$

für $x=0$ :

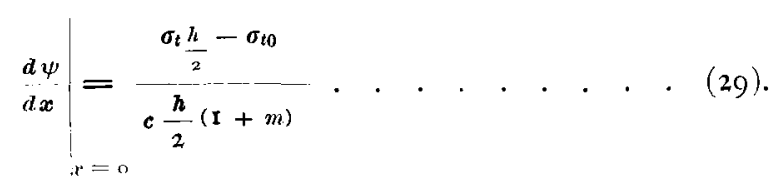

Sind also für $\sigma_{w}$ tund $\sigma_{\frac{t}{2}} \frac{h}{2}$ die ersten Schätzungen getroffen, so kann danach für das erste Intervall $l x$ das $\frac{d \psi}{d x}$ berechnet werden, und damit sind auch für die letzte der drei Hauptgleichungen, d. i. fiir (III a) alle Unterlagen geschaffen.

2. Fall: Die Platte hat in der Mitte eine kreisrunde Bohrung vom Halbmesser $x_{i}$.

Wine einfache Ueberlegung zeigt, daß für

also auch

$$
\begin{aligned}
x=x_{i} \quad \sigma_{r 0 i} & =0 \\
\sigma_{r \eta i} & =0, \\
\sigma_{r \frac{h}{2} i} & =0 .
\end{aligned}
$$

Fïr das zugehörige $\sigma_{t 0 i}$ muß ein erster Wert abgeschäitzt werden; hierfïr

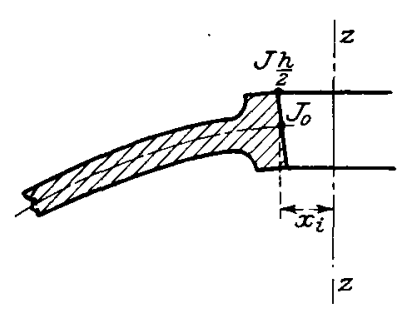

Fin. Io. sind die gleichen Frwägungen und Hülfsmittel zı empiehlen, wie im Fall r. Nur int hier fül $\sigma_{t u i}$ gegenüber dem Mittelwert $\sigma_{t}^{*}$ in der Regel noch cin weit größerer Zuschlag zu machen, insbesonder' dann, wenn dem Einfluß der Bohrung nicht durch eine kräftige Versteifung des inneren Scheibenrandes: in Form einer Nabe begegnet wird, wie dies in Fig. Io schematisch dargestellt ist. Unter Berücksichtigung des eingangs dieses Abschnittes Gesagten geht

Gl. (3) fïr den besonderen Fall, dab $x=x_{i}$ und $y={ }_{2}^{h}$, iiber in die Form

ঐemnach muß fïr $x=x_{i}$

$$
\circ=\circ+c \frac{h}{2} \cos \varphi\left[m \frac{d \psi}{d x}+\frac{\psi}{x}\right]
$$

$$
\begin{aligned}
& \frac{d \psi}{d x}=-\frac{\psi}{m \frac{\psi}{x_{i}}} \text {. . . . . . . . } \\
& \text { t in Gl. (4), gibt für } x=x_{i} \text { und } \eta=\frac{h}{2}
\end{aligned}
$$

fiir $x=x_{i}$

$$
\begin{aligned}
\sigma_{t} u_{i} & =\sigma_{t 0 i}+c \frac{h}{2} \cos \varphi\left[-\frac{\mathrm{I}}{m} \frac{\psi}{x_{i}}+m \frac{\psi}{x_{i}}\right] \\
& =\sigma_{t(t i}+c \frac{h}{2} \cos \varphi\left[\frac{m^{2}-1}{m} \frac{\psi}{x_{i}}\right]
\end{aligned}
$$

sein. Diese Beziehung, eingesetzt in Gl. (4), gibt fiir $x=x_{i}$ und $\eta=\frac{h}{2}$

$$
\psi_{i}=\left[\begin{array}{c}
\sigma_{t h_{i}}-\sigma_{t i) i} \\
\frac{1}{2}
\end{array}\right]\left(\begin{array}{c}
m x_{i} \\
m^{2}-\mathrm{I}
\end{array}\right) \frac{\mathrm{I}}{c \frac{h}{2} \cos \gamma} .
$$


Sobald wir also für $x=x_{i}$ außer dem für den Punkt $J_{0}$ in Fig. Io gültigen Wert $\sigma_{t a i}$ noch den Wert $\sigma_{\frac{t}{2} h_{i}}$ für $\frac{J h_{i}}{2}$ abschätzen, liefert die letzte Gleichung den zugehörigen Wert für die Winkeländerung $\psi_{i}=\Delta \varphi_{i}$.

Berücksichtigt man überdies, daß wir früher setzten $c=\frac{m E}{m^{2}-\mathrm{I}}$, so geht diese letzte Gleichung iiber in die etwas einfachere Form

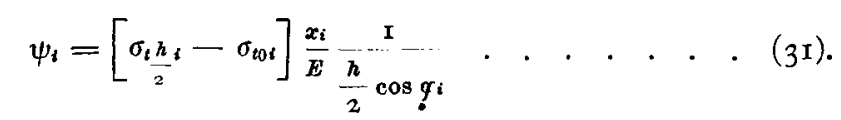

Damit sind alle Unterlagen geschaffen für Anwendung der Hauptgleichungen (I), (II), (III) oder (Ia), (II a) und (IIIa).

Wir können nunmehr die Radial- und Tangentialspannungen und die Winkeländerung in allen Punkten des Plattenquerschnittes vom inneren Rand bis zur Auflage und der Einspannstelle am äußeren Rand berechnen.

\section{Randbedingungen.}

In Anlehnung an die Radscheibenberechnung wollen wir hierunter die Bedingangen verstehen, unter welchen die Platte am äußeren Rand unterstïtzt ist, und besonders die beiden Fälle herausgreifen, in denen die Platte am Außenrand frei aufliegt oder fest eingespannt ist. Um Verwechslungen vorzubeugen, möge mit der Numerierung der "Fälle* einfach weiter gefahren werden.

3. Fall: Die Platte liegt am äußeren Rand frei auf, Fig. II.

Die Mittelfaser des Meridianschnittes endige im Halbmesser $x_{a}$. Wir betrachten die Auflagefläche der Platte als Mantel eines Kegelstumpies, dessen Fr-

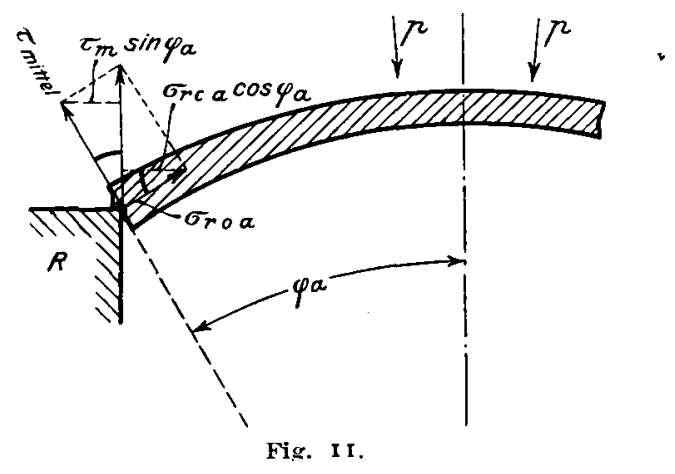

zeugende die Länge $h_{a}$ haben. Sie erleidet in Richtung der Symmetrieachse $z-z$ einen Gesamtschub von der Größe

$$
\Sigma P_{a}^{\prime}=\left(x_{a}^{2}-x_{i}^{2}\right) \pi p .
$$

Diese Kraft erzeugt im Auflagepunkt des Plattenrandes, Fig. Ir, in Richtung der Meridian-Mittelfaser eine mittlere Radialspannung $\sigma_{\text {roa }} . \mathrm{Zu}$ deren Berechnung führt lolgende: Weg: Wir denken uns in der Mitte des äußeren BegrenzungsKegelmantels ringsum eine Kerbe eingedreht, deren eine Fliache eben und zur Symmetrieachse senkrecht gerichtet ist. Mit dieser Ebene liegt die Platte auf einem im Raum starren Ring $R$ auf. Der Ring $R$ kann nur Kräfte parallel zur Symmetrieachse, nicht aber senkrecht dazı gerichtete Kräfte und auch kein Moment auf die Platte übertragen. Die für $\mathrm{I} \mathrm{cm}$ des Umfanges vom Wider- 
lagerring $R$ auf die Platte übertragene Kraft kann zerlegt werden in zwei Komponenten:

I) in die Normalkraft $=\sigma_{r, a} h_{a} \mathrm{I}$.

2) in die Schubkraft $=\boldsymbol{\tau}_{\text {mittel }} \boldsymbol{h}_{l} \mathrm{I}$.

Weil der Ring $\boldsymbol{k}$ keine Kraftkomponente in Richtung senkrecht zur Symmetrieachse ausüben kain, so muß die Summe der in die Richtung des Halbmessers $x$ entfallenden Komponenten der unter r) und 2) genannten Kräfte gleich null sein.

Wir finden hierfür an Hand der Fig. I I folgende Gleichung:

demnach

$$
\left(\tau_{\text {mittel }} h_{a} \mathrm{I}\right) \sin \tau_{a}-\left(\sigma_{r 0 a} h_{a} \mathrm{I}\right) \cos \tau_{a}=0 \text {, }
$$

$$
\tau_{\mathrm{mittel}}=\sigma_{r 0 a} \frac{\cos \gamma_{a}}{\sin \varphi_{a}} .
$$

Der ganze ling $R$ übt auf die Platte eine Widerlagerkraft aus:

$$
\left[\left(\tau_{\text {mittel }} h_{a} \mathrm{I}\right) \cos \gamma_{a}+\left(r_{r 0 a} h_{n} \mathrm{I}\right) \sin \Upsilon_{a}\right] 2 \pi x_{a} .
$$

Sie ist gleich der Krait, welche der iußere Ueberdruck $p$ in "Richtung der Symmetrieachse auf die l'latte ausübt.

Wir finden also:

$$
\left[\tau_{\text {mittel }} h_{a} \cos \Upsilon_{a}+\sigma_{r_{0} a} h_{a} \sin \Upsilon_{a}\right] 2 \pi x_{a}=\left(x_{a}{ }^{2}-x_{i}{ }^{2}\right) \pi p . \quad . \quad \text { (32). }
$$

Aus der vorhergehenden Gleichung substituieren wir den Wert für $\boldsymbol{\tau}_{\text {mittel }}$ und erhalten:

$$
\begin{aligned}
& 2 \pi x_{a} h_{a}\left(\sigma_{r 0 a} \frac{\cos ^{2} \varphi_{a}}{\sin q_{a}}+\sin \tau_{a}\right)=\left(x_{a}{ }^{2}-x_{i}{ }^{2}\right) \pi p .
\end{aligned}
$$

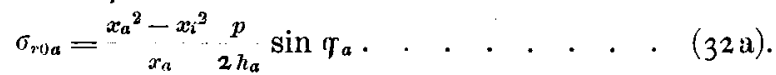

Ist am Außenrand der Platte die Meridian-Mittelfaser normal zur Symmetrieachse $z-z$ gerichtet, also $\varphi_{a}=0$, so ist laut Gl. (32a) auch $\sigma_{r 0 a}=0$.

Als zweite Randbedingung gilt für den Fall 3), wo die Platte am Rand frei aufliegt, die Bedingung, dal3 im äußersten Querschnitt keine Biegungsspannungen herrschen dürfen, daß also dort das Moment der inneren Spannungen

$$
\boldsymbol{M}_{\boldsymbol{\sigma}_{r a}}=0
$$

sein mul. Dies ist der Fall, wenn in Gl. (20) für

wenn also für

$$
\boldsymbol{x}=\boldsymbol{x}_{\boldsymbol{a}} \ldots\left[m \frac{\boldsymbol{d} \psi}{\boldsymbol{d} \boldsymbol{x}}+\frac{\psi}{x}\right]=0 . . . \quad . \quad . \quad \text {. (33), }
$$

$$
x=x_{a} \ldots m \frac{d \psi}{d x}=-\frac{w}{x} \text {. . . . . . . . (33a). }
$$

Es müssen nun für $x=x_{i}$ die Werte $\sigma_{t 0 i}$ und $\sigma_{\frac{t}{2} i}$ solange geändert, und für jedes Wertepaar muß die ganze Intervallrechnung durchgeführt werden, bis die in den Gl. (32a) und (33) ausgedrückten Randbedingungen mit hinreichender Annäherung in Erfüllung gehen.

Am besten geht man dann so vor, daß man in einem Dreikoordinatensystem, z. B. in Richtung der $x$-Achse die für die verschiedenen Durchrechnungen angenommenen Werte $r_{t 0 i}$, in Richtung der $y$-Achse die Werte $\sigma_{\frac{t}{2}} h_{i}$, aufträgt und sodann senkrecht über dem durch zwei zusammengehörige Werte fixierten Punkt der $(x-y)$-Ebene einmal den Wert $\sigma_{r a a}^{\prime}$ und den Klammeraus- 
druck $\left[m \frac{d \psi}{d x}+\frac{\psi}{x}\right]$ in passendem Maßstab bildlich darstellt. Weicht $\sigma_{r_{0 a}^{\prime}}$ ab vom Wert $\sigma_{m a}$, der durch Gl. (32a) festgelegt ist, und wird der Klammerausdruck [] nach Gl. (33) für $x_{a}$ nicht gleich null, so sind für $\sigma_{t 0 i}$ und $\sigma_{\frac{t}{2}}{ }_{i}$ neue

Annahmen zu treffen. Das graphische Bild im Dreikoordinatensystem zeigt den Weg zu einer zweckmäßigen Interpolation.

4. Fall: Die Platte ist außen eingespannt. Hierzu Fig. I2.

Der allgemeinere Fall wäre nun der, daß man annimmt, die die Platte haltende Wandung sei für sich auch drehbar. Das würde aber zu einer endlosen Rechnung führen. Wir wollen uns daher auf den etwas.engeren Fall beschränken, der Querschnitt der zylindrischen Außenwandung sei nicht nach innen oder außen drehbar, sondern ändere unter dem Einfluß der Platte einerseits und des Ueberdruckes $p$ anderseits lediglich seinen Durchmesser, und zwar ist die Aenderung dieses Durchmessers gleich der Aenderung des Außendurchmessers $\left(2 x_{n}\right)$ der Platte.

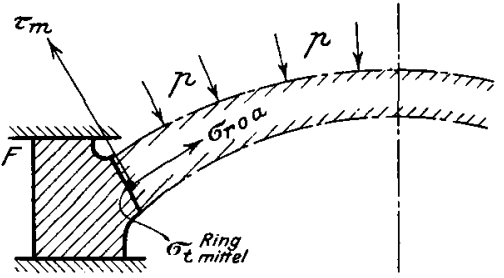

Fig. I2.

Daß die Platte an der Uebergangstelle zum zylindrischen Mantel nicht drehbar eingespannt sei, läßt sich durch die Bedingungsgleichung ausdrücken

für $x=x_{a} \ldots \psi_{a}^{\prime}=0$. . . . . . . . (34).

Zwischen der im Stützring sich bildenden mittleren Tangentialspannung $\sigma_{i \mathrm{ittel}}$ und den an der Uebergangstelle von der Platte zum Ring herrschenden mittleren Normal- und Schubspannungen, d. i. $\sigma_{r 0 a}$ bezw. $\tau_{\text {mittel, }}$ lassen sich folgende Beziehungen aufstellen:

Der Ring habe einen Querschnitt $F$, dann ist

$$
\begin{aligned}
& 2 \sigma_{\text {mittel }} F=2 x_{a} h_{a}\left(\sigma_{\text {raa }} \cos \varphi_{a}-\tau_{\text {mittel }} \sin \varphi_{a}\right) \\
& \underset{\text { im Stützring }}{\sigma_{\text {mittel }}=}=\frac{x_{a} h_{a}}{F}\left(\sigma_{r 0 a} \cos \varphi_{a}-\tau_{\text {mittel }} \sin \varphi_{a}\right) \quad . \quad . \quad . \quad . \quad \text {. } \quad \text { (35). }
\end{aligned}
$$

Der Zusammenhang zwischen $\sigma_{r a n}$ und $\tau_{\text {mittel }}$ am Plattenrand geht aus G1. (32) hervor.

Unter dem Einfluß der Tangentialspannung $\sigma_{\text {mittel }}$ dehnt sich der mittlere Halbmesser des inneren Begrenzungskegels vom Stützring, d. i. $x_{a}$, um die Strecke

$$
\Delta x_{a}^{\prime}=x_{a}^{\prime} \frac{\sigma_{\text {mittel }}}{E} .
$$

Der Außenhalbmesser $x_{a}$ der Platte erfährt eine Dehnung

$$
\Delta r_{a}^{\prime \prime}={ }_{E}^{x_{a}}\left(\sigma_{t 0 a}-\frac{\sigma_{r 0 a}}{m}\right)
$$

Unter der Bedingung, daß die Platte sich vom Ring nicht lösen und ihm gegenüber nicht vel'schieben darf, muß sein

also

$$
\Delta x_{a}^{\prime}=\Delta x_{a}{ }^{\prime \prime},
$$

für den Ring: für die Platte:

$$
\sigma_{\text {mittel }}=\left(\sigma_{t 0 a}-\frac{\sigma_{r 0 a}}{m}\right) . . \quad . \quad . \quad . \quad\left(3^{6}\right)
$$


Die aus den (1). (35) und (36) berechneten Werte für $\sigma_{\text {mittel }}$ des Ringes mïssen miteinander übereinstimmen. Bis dies der Fall und die Bedingungsgleichung (34) erfiullt ist, muß mit dem Wertepaar $\sigma_{t 0 i}$ und $\sigma_{t} h_{i}$ variiert werden.

5. Fall: Die Platte ist am Rande so befestigt, daß ihr Außendurchmesser sich nicht ändern kann.

Dies drückt sich aus durch die Bedingung: $\Delta x_{a}=0$; dann ist auch $\frac{\Delta x_{a}}{x_{a}}=0$ und weil $\frac{\Delta x_{a}}{x_{a}}=\varepsilon_{t 0 a}$, so muß auch $\varepsilon_{t 0 a}=0$ sein.

Nun ist laut Elastizitätslehre $\left.{ }^{1}\right)$ :

$$
\varepsilon_{t 0 a}=\frac{\mathbf{I}}{\boldsymbol{E}}\left(\sigma_{t 0 a}-\frac{\sigma_{r_{n} n}}{m}\right) .
$$

$\varepsilon_{t 0 a}$ kann nur $=0$ werden, wenn

$$
\sigma_{i 0 a}={ }_{m}^{\sigma_{0 a}} \text {. . . . . . . . . . . . } .(36 \mathrm{a}) .
$$

Diese Begingungsgleichung dafür, daß der Außendurchmesser $x_{a}$ unver:indert bleibe, hat ihre Gültigkeit ganz unabhängig davon, ob die Platte am Rand so gestützt ist, daß ihr äußerster Querschnitt sich noch drehen kann $\left(\psi_{a}\right.$ nicht $\left.=0\right)$ oder zugleich so eingespannt ist, daß dies nicht möglich $\left(\psi_{a}=0\right)$, wie dies als " 4 . Fall " behandelt wurde.

Um zu diesen vorläufigen Schlußergebnissen zu gelangen, haben wir uns bisher nur der Hauptgleichungen (Ia), (IIa) und (III a) bedient und uns also stillschweigend nur um die Normalspannungen in der Nittelfaser gekümmert.

Ist erst ein Wertepaal $\sigma_{t 0 i}$ und $\sigma_{t_{i}}$ so gefunden, daß die sich hierauf stützende Durchrechutung der Gl. (I) bis (III) von Intervall zu Intervall die Randbedingungen erfüllt, so können mit Hïlfe der Gl. (3) und (4) für jeden im Abstand $\eta$ von der Meridianmittelfaser liegenden Punkt die Radialspannung $\sigma_{r}$ und die 'Tangentialspannung $\sigma_{t}$ berechnet werden. Man wird sich jedoch darauf beschrünken, dies für die auf der äußeren und inneren Plattenoberfläche gelegenen Punkte zu tun, deren Abstand von der Mittelfaser

$$
\eta= \pm_{2}^{h} \text { ist. }
$$

Zwischenliegende Werte ändern sich in einem linearen Verhältnis mit dem Abstand $\eta$.

Trägt man in einem axonometrischen Bild den Meridianschnitt durch die Platte und senkrecht dazu einmal die Radial-, sodann die Tangentialspannung auf, so erhält man ein äußerst anschauliches Bild von dem Verlauf der spezifischen Beanspruchungen über den ganzen Plattenquerschnitt. Dieses Bild wird zeigen, wo in der Platte schwache Stellen sind, wo sie verstärkt werden sollte, wo andererseits an Material gespart werden dürfie, um die Platte einem Körper gleicher Festigkeit bustmöglich anzupassen.

Natürlich kann jeder der soeben behandelten Fílle I) und 2) mit jedem der Fialle 3) bis 5) vereingt werden, so daß also unser Verfahren die Berechnung von Platten zuliilst, die in der Mitte voll oder durchbrochen und am Rand frei aufliegend oder eingespannt und so gestützt sind, daß sich ihr Außenhalbmesser $\left(x_{a}\right)$ ändern kann oder nicht.

1) s. Föppl 1909 Band III S. 246. 


\section{Durchbiegung der gewölbten Platte.}

Haben wir im Vorstebenden für jeden Punkt des Plattenquerschnittes die Radial-, die Tangentialspannung und die Winkeländerung berechnet, so sind wir damit in der Lage, die Durchbiegung ermitteln zu können. Es genügt, wenn wir hierbei nur die Mittelfaser in Betracht ziehen, welche der Radialspannung $\sigma_{r 0}$ und der Tangentialspannung $\sigma_{10}$ ansgesetzt ist.

Wir schlagen denselben Weg ein, den v. Bach für die Ermittlung der Durchbiegung eines gekrümmten Balkens gezeigt hat ${ }^{1}$.

In Fig. I3 sei durch den Linienzug $J-A$ lediglich die Mittelfaser einer gewölbten Platte dargestellt. Aus ihr greifen wir ein beim Punkt $\boldsymbol{P}$ gelegenes Element von der Länge $d s$ heraus.

Wir berechnen vorerst die Verschiebung des zweiten Endpunktes $C$ dieses Elementes gegenüber dem Punkt $P$. Während der $\mathrm{Be}-$ lastung der Platte durch die äußere spezifische Spannung $p$ gelangt das Faserelement aus der Lage $P-C$ vom unbelasteten Zustand in die Lage $P^{\prime}-C^{\prime \prime}$ des endgültig belasteten $\mathrm{Zu}$ -

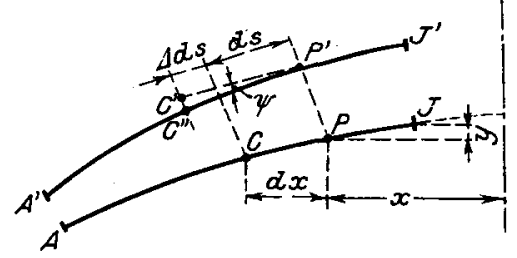

Fig. I 3. standes. Diese Veränderung kann als in zwei Phasen ausgeführt gedacht werden:

I) Verschiebt sich das Element aus der Lage $P-C$ zu sich selbst parallel und dehnt sich unter dem Einfluß der Längsspannung $\sigma_{r 0}$ und der Querspannung $\sigma_{t 0}$ um den Betrag

$$
J(d s)=\frac{d s}{E}\left(\sigma_{r 0}-\frac{\sigma_{t 0}}{m}\right)
$$

Hierbei gelangt es in die Lage $P^{\prime}-C^{\prime}$.

2) Dreht es sich aus dieser Lage um den Winkel $\psi$ in die Lage $P^{\prime}-C^{\prime \prime}$. Wie wir schon bei Berechnung der Spannung $\sigma_{t v}$ sahen, ändert sich hierbei die $\boldsymbol{x}$-Koordinate des Punktes $C$ gegenüber dem Punkt $P$ um den Betrag

$$
\begin{aligned}
& J(d x)=\Delta(d l s) \cos \varphi-C^{\prime} C^{\prime \prime} \sin \varphi \\
& =\frac{d \boldsymbol{s}}{E}\left(\sigma_{r 0}-\frac{\sigma_{t 0}}{m}\right) \cos \varphi-d s \psi \sin \varphi \quad .
\end{aligned}
$$

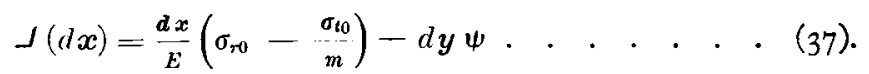

Die $y$-Koordinate des lunktes $C$ ändert sich gegenüber dem Punkt $P$ um den Betrag

$$
\begin{aligned}
& J(d y)=J(d s) \sin \varphi+C^{\prime} C^{\prime \prime} \cos \varphi \\
& ={ }_{E}^{d \boldsymbol{s}}\left(\sigma_{r 0}-\frac{\sigma_{t 0}}{m}\right) \sin \varphi+d s \psi \cos \varphi
\end{aligned}
$$

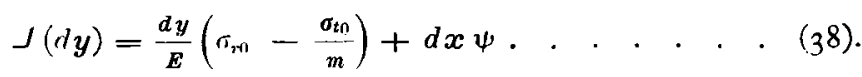

(Hierbei setzen wir als Regel, die $y$-Koordinate in Fig. I 3 von der Höhe des Punktes $J$ aus von oben nach unten positiv zu zählen.)

Der Punkt $C$ ändert während der allmählich vor sich gehenden Durchbiegung der Platte seine Koordinaten gegenüber dem Punkt $J$ um die Werte

$$
\begin{aligned}
& \Delta x_{J}^{*}=2 j J(d x) \quad . \quad . \quad . \quad . \quad . \quad . \quad . \quad . \quad \text { (39) }
\end{aligned}
$$

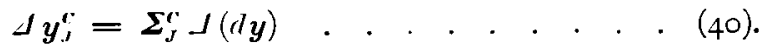

(iemäi unserer bisher geübten Rechnungsweise setzen wir statt der unendlich kleinen Differentiale $d x$ und $d y$ die endlich kleinen Intervalle $d x$ und $d y$ ein.

1) s. Bach »Elastizitäts- und Festiekkeitslehre« I9O2 S. 485 . 


$$
-24-
$$

Will man die Aenderung der $x$-Ordinate des Punktes $C$ in bezug auf die Symmetrieachse $z-z$, Fig. I 3 , berechnen, so ist zu berücksichtigen, daß die Ordinate des Punktes $J$ sich bereits ändert um den Betrag

$$
\left.\Delta \boldsymbol{x}_{J}=\frac{\boldsymbol{x}_{J}}{\boldsymbol{E}}\left(\sigma_{\mathrm{t} 0}-\frac{\sigma_{r 0}}{m}\right) \text {. . . . . . . . (4 } \mathrm{I}\right) .
$$

Dieser Wert ist zu dem in Gl. (39) aufgestellten Wert zu addieren, um dic Gesamtzunahme der $x$-Ordinate des Punktes $C$ gegenïber der Symmetrieachse $\mathrm{zu}$ erhalten.

Wir finden also für diesen Punkt

$$
\Delta x_{C}=\left\{\begin{array}{l}
\Sigma_{J}^{c}\left[\frac{d x}{E}\left(\sigma_{r 0}-\frac{\sigma_{t 0}}{m}\right)\right] \\
-\Sigma_{J}^{c}[\psi d y] \\
+\frac{x_{J}}{E}\left(\sigma_{t 0}-\frac{\sigma_{+0}}{m}\right)_{x=x_{J}}
\end{array} . \quad . \quad . \quad . \quad . \quad\right. \text { (42). }
$$

Hat die Platte in der Mitte kein Loch, so fällt der letzte Summand natürlich weg, weil dann $x_{J}=0$.

$$
\Delta y_{c}=\Sigma_{J}^{c}\left[\frac{d y}{E}\left(\sigma_{, 0}-\frac{\sigma_{t_{0}}}{m}\right)\right]+\Sigma_{J}^{c}(\psi d x) . . . \quad . \quad(43) .
$$

Man schafft sich ein anschauliches Bild von der Formänderung der Platte, wenn man die Koordinatenänderung eines jeden Punktes der Meridian-Mittelfaser in viel größerem, und zwar dem 20- bis Ioofachen Maßstab aufzeichnet gegenüber den Koordinaten selbst.

\section{Zahlenbeispiele.}

Das entwickelte Rechnungsverfahren soll num angewendet werden aut einige Zahlenbeispiele:

Wir wählen als erstes Beispiel den in Fig. I4 dargestellten gewölbten gußeisernen Deckel, wie ein solcher in den Werkstiitten von Escher, Wyß \& Cie.

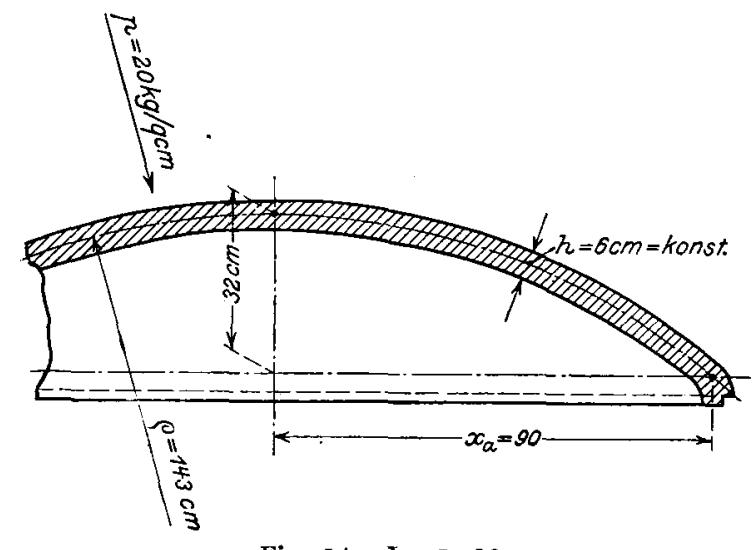

Fig. I4. I. I: 20.

in Zürich dazu verwendet wird, die Gehäuse von Dampiturbinen probehalber abzupressen. Es ist dies also ein Hülfsboden, der nicht zur Lieferung der 'Turbine gehört.

Der Boden ist gemäß Fig. I4 in der Mitte voll, hat eine gleichmäßige Wölbung mit einem Krümmungshalbmesser $\varrho=143 \mathrm{~cm}$ und soll laut Werkstattzeichnung eine stets gleiche Dicke von $h=6 \mathrm{~cm}$ haben. 
Wir haben diese gewölbte Platte als Beispiel gewählt, weil sie als Teil einer Hohlkugel vom Halbmesser $\varrho=I 43 \mathrm{~cm}$ und von stets gleicher Dicke einen wertvollen Vergleich mit der vollständigen Hohlkugel für den Fall gleicher spezifischer Belastung zuläßt.

Die nachstehende Rechnung wurde durchgeführt für einen auf der konvexen Seite wirkenden Ueberdruck von 20 at

$$
p=(-20) \mathrm{kg} / \mathrm{q} \mathrm{cm} \text {. }
$$

Dieser Druck wurde für die Rechnung so hoch gewïhlt, um sie zuverläßlicher zu gestalten. Für kleinere Ueberdrücke ändern sich die Ergebnisse proportional.

$$
\text { Vergleich mit der Hohlkugel. }
$$

Bei $(-20)$ at Ueberdruck würde die vollständige Hohlkugel vom mittleren Ifalbmesser $\varrho=143 \mathrm{~cm}$, der Dicke $h=6 \mathrm{~cm}$ eine mittlere Druckspannung $\sigma_{k}$ erfahren, die sich nach der Formel berechnen läßt:

$$
\begin{aligned}
& \sigma_{k}=\left(R^{2} \pi p\right):(2 R \pi h)=\frac{R p}{2 h}, \\
& \sigma_{k}=\frac{143 \cdot(-20)}{2 \cdot 6}=-239 \mathrm{~kg} / \mathrm{qcm} . . \quad . \quad . \quad . \quad(44) .
\end{aligned}
$$

Unter dieser spezifischen Belastung würde sich der Halbmesser verändern un den Betrag

$$
\Delta R=\frac{R}{E}\left(\sigma_{t k}-\frac{\sigma_{r k}}{m}\right) .
$$

Hierin ist $R=\mathrm{I}_{43} \mathrm{~cm} ; E$ für Gußeisen $=900000 \mathrm{~kg} / \mathrm{qcm} ; m$ für dieses Material rd. $\left.5^{1}\right) ; \sigma_{t k}=\sigma_{r k}=\sigma_{k}=-239 \mathrm{~kg} / \mathrm{qcm}$.

$$
\begin{aligned}
& \Delta R=\frac{143}{900000}(1-1 / 5)(-239)=-0,0305 \mathrm{~cm}, \\
& \Delta R=-0,305 \mathrm{~mm} \text {. . . . . . . . (45). }
\end{aligned}
$$

Wie bereits bemerkt, ist es nun höchst lehrreich, festzustellen, wie sich die Hohlkugelkalotte in ihrer Beanspruchung und Formänderung der vollständigen Hohlkugel gegenüber verhält.

Untersuchung der Platte nach Fig. It für $p=(-20) \mathrm{kg} / \mathrm{qcm}$.

Diese Platte von der Form einer Hohlkugelkalotte hat einen äußeren Halbmesser

$$
x_{a}=90 \mathrm{~cm} \text {. }
$$

Weil sie in der Mitte nicht durchbrochen, so ist

$$
\boldsymbol{x}_{i}=\text { o. }
$$

Der Boden liegt bei der Verwendung auf einer im Turbinengehäuse vor gesehenen Randfläche auf, welche einer Ebene angehört, die zur Turbinenachse also zur Symmetrieachse der Platte senkrecht steht. Diese Auflage vermag demnach auf die Platte nur Reaktionskräifte in Richtung parallel zur Symmetrieachse zu übertragen.

Der Meridianquerschnitt erfïhrt laut dem auf $\$ .47$ Gesagten eine mittlere Tangentialspannung

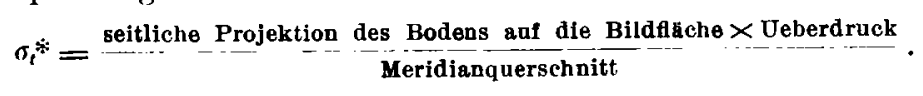

Die seitliche Projektion des Bodens auf die Bildfläche berechnet sich wie folgt: (vergl. Fig. I)

1) siehe Föppl Bd. III S. 43 Zeile 5 von unten. 


$$
\begin{aligned}
& \text { Fläche } A_{1} J A_{2} J_{1} A_{1}=F=\frac{\varrho^{2}}{2}\left(2 \frac{\gamma_{a}{ }^{0} \pi}{180^{0}}-\sin \left(2 \gamma_{a}^{0}\right)\right) \text {, } \\
& \sin \varphi_{a}{ }^{0}=\frac{x_{a}}{p}=\frac{90}{I_{43}}=0,63 \text {, } \\
& \boldsymbol{q}_{a}{ }^{0}=39^{0} ; \tilde{\boldsymbol{q}_{a}}=0,68 \mathrm{I} \\
& \sin \left(2 \varphi_{a}^{\circ}\right)=\sin 78^{\circ}=0,978 \text {, } \\
& 2 \frac{\varphi_{a}^{0} \pi}{180^{0}}=2 \widehat{\varphi_{a}}=\mathrm{I}, 362 . \\
& F=\frac{143^{2}}{2}(\mathrm{I}, 362-0,978)=3920 \mathrm{qcm} . \\
& f=2 \cdot 0,68 \mathrm{I} \cdot \mathrm{I} 43 \cdot 6=\mathrm{II} 70 \mathrm{qcm} . \\
& \sigma_{t}^{*}=\frac{F p}{r}=\frac{3920(-20)}{\mathrm{I} 70}=(-67) \mathrm{kg} / \mathrm{qcm} . . .
\end{aligned}
$$

statt $\sigma_{k}=(-239) \mathrm{kg} / \mathrm{qcm}$, wie wir in Gl. (44) für :die vollständige Hohlkugel gefunden hatten. Diese Verschiedenheit läßt bereits vermuten, daß in dem zu berechnenden Boden die Beanspruchung von Punkt zu Punkt der Mittelfaser (und in noch höherem Maß der Außenfaser) stark veriinderlich sein werde. Es ist nämlich anzunehmen, daß die Beanspruchung des Bodens in der Nähe der Symmetrieachse von derjenigen in der vollen Hohlkugel nicht sehr abweicht. Damit aber die mittlere Beanspruchung des Bodens, d. i. $\sigma_{t}^{*}$ nur etwa $1 / 4$ (d. i. "-67" gegeniiber »-239") von derjenigen der Hohlkugel sei, muß am Außenrand eine große Beanspruchung auftreten, welche ein entgegengesetztes Vorzeichen zu derjenigen in der Mitte des Bodens hat. Die in den nachstehenden Figuren und in Zahlentafel 8 wiedergegebenen Rechnungswerte bestätigen denn auch die Richtigkeit dieser Vermutung.

Die außen frei aufliegende Platte erfährt daselbst laut Gl. (32a) eine mittlere sogenannte Radialspannung

$$
\begin{gathered}
\sigma_{r a}=\frac{x_{a} p \sin \varphi_{a}}{2 h_{a}}=\frac{90 \cdot(-20) \cdot 0,63}{2 \cdot 6} \\
\sigma_{r a}=(-94) \mathrm{kg} / q \mathrm{~cm} \ldots \text {. . (Druckspannung) . . . . (47). }
\end{gathered}
$$

Nunmehr stellen wir uns das Schema für die Hauptzahlentafel auf:

Dabei beginnen wir nicht mit $x_{i}=0$, wie wir dies eigentlich tun soliten, sondern mit $x=$ ro $\mathrm{cm}$. Wir müßten nämlich sonst die ersten Intervalle $d x$ so klein nehmen, damit sie gegenüber $x$ vorschriftsmäßig klein genug sind, daß dadurch die Rechnung sehr weitliutifg würde, ohne besonderen Gewinn zu ergeben. So schätzen wir die zu machenden Annahmen statt für $x=0$ für $x=1 \circ \mathrm{cm}$ und extrapolieren nach erfolgter Durchrechnung rückwärts auf $x=0$.

Vom Halbmesser $x=10 \mathrm{~cm}$ ausgehend, wählen wir die Intervalle $d x$ zuerst nur klein $(d x=\mathrm{x})$, dann immer größer, bis zuletzt $d x=5 \mathrm{~cm}$ wird und bleibt. (Wie sich jedoch erst später herausstellte, wäre es für die Genauigkeit der Rechnung besser gewesen, das Intervall $d x$ gegen den Außenrand hin wieder kleiner werden zu lassen, z. B. $d x=5,4,3,2 \mathrm{~cm}$.) Für welche Halbmesser die Einzelrechnungen auszuführen sind, muß eben das praktische Gefühl und etwas Uebung weisen. In unserem Beispiel wurden sie vorgenommen für

$$
\begin{array}{r}
x=\mathrm{I0}, \mathrm{I} \text { I, I2, I3, I4, 16, I8, 20, 22, 24, 26, 29, 32, 36, 40, 45, 50, } \\
55,60,65,70,75,80,85 \text { und } 90 \mathrm{~cm} .
\end{array}
$$

Zahlentafeln I bis 4 zeigen als Ausführungsbeispiel den Kopf und wenigstens den oberen Teil der sogenannten "Haupttafel«. Die einzelnen Spalten sind gekennzeichnet durch fortlaufende, eingeklammerte Nummern. In jeder Spalte ist in deren Kopf schematisch dargestellt, ob sie aus früheren Spalten entstanden, aus welchen und in welcher Weise. 


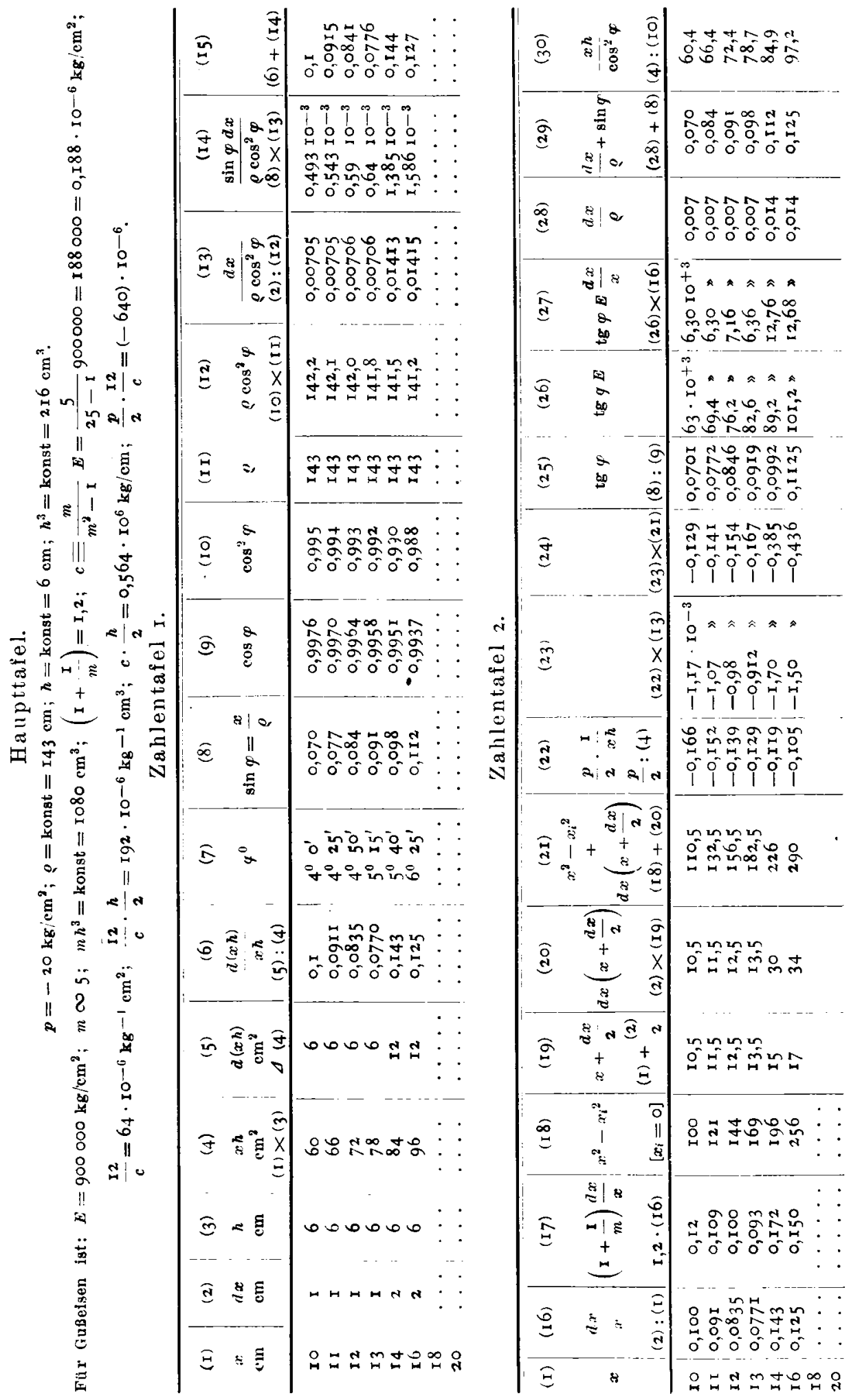



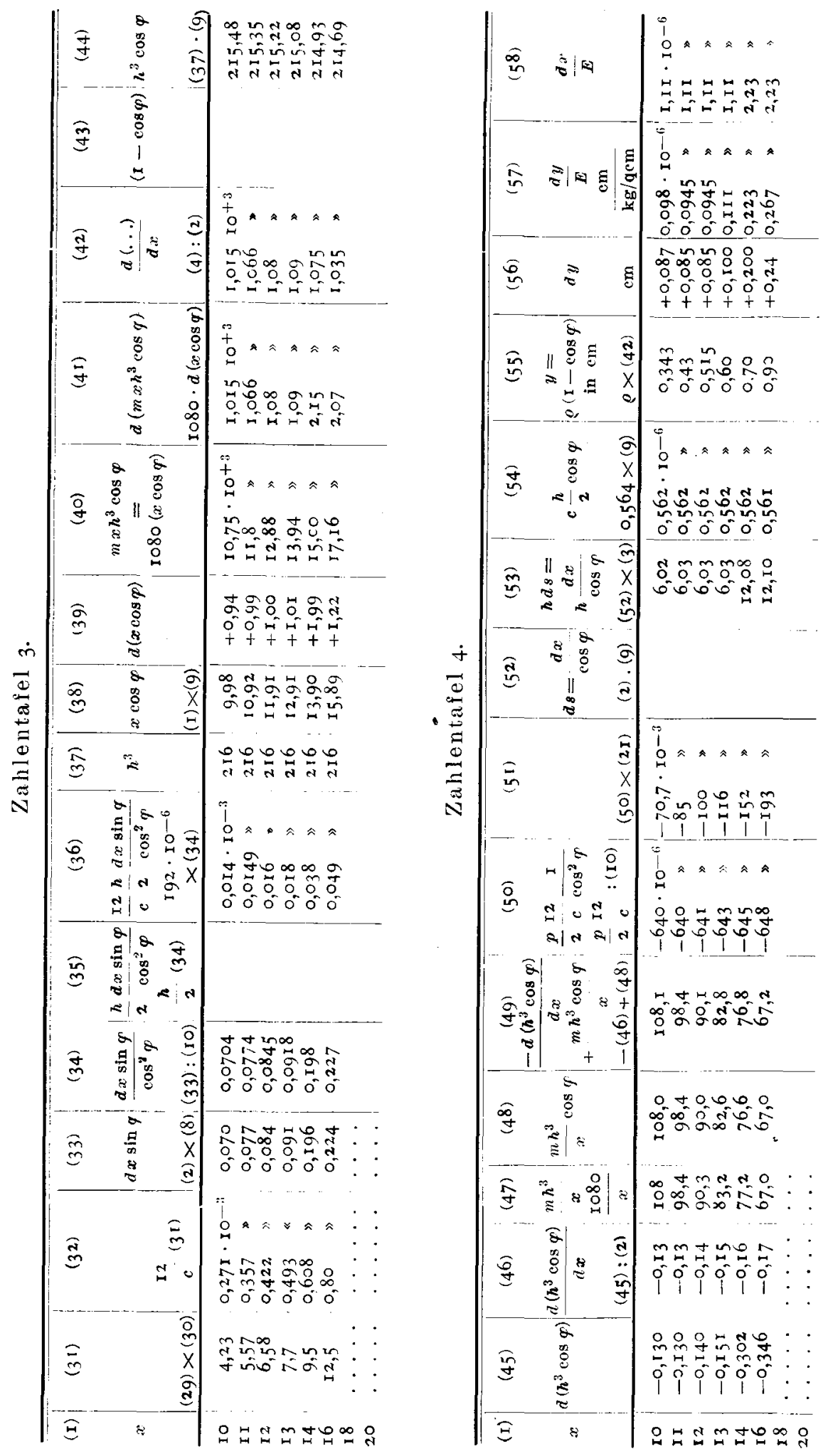
Des weiteren wird in den naichsten Zallentafeln 5 bis 7 ebenfalls nur der Beginn eines sogenannten "Ausrechnungsblattes wiedergegeben, und zwar sind erst die Konstanten aus der "Haupttafel ", aber noch keine Ausrechnungswerte cingetragen. Für letztere sind vielmehr erst .... eingetragen.

Vergleichshalber wurden die beiden naheliegenden Fälle durchgerechnet: Fall 3): Platte am Rand frei aufliegend,

4): » $~ »$ eingespannt,

und zwar beide in Kombination mit Fall $\mathrm{r}$ ): Platte innen voll.

Zahlentafel 5. (Ausrechnungsblatt.)

\begin{tabular}{|c|c|c|c|c|c|c|c|c|c|c|}
\hline $\begin{array}{l}\text { (I) } \\
x\end{array}$ & $\begin{array}{c}(8 \mathrm{I}) \\
(\mathrm{I} 5) \cdot \sigma_{r_{0}}\end{array}$ & $\begin{array}{c}(82) \\
(I 6) \cdot \sigma_{t_{0}}\end{array}$ & $\begin{array}{c}(83) \\
d \sigma_{r_{0}}= \\
+(24) \\
-(81) \\
+(82)\end{array}$ & $\begin{array}{c}(84) \\
\sigma_{r_{G 2}}= \\
\sigma_{r_{01}}+d \sigma_{r_{0}}\end{array}$ & $\begin{array}{c}(85) \\
\sigma_{r_{0}}-\sigma_{t_{0}}\end{array}$ & $\begin{array}{l}\quad(86) \\
\left(\mathrm{I}+\frac{\mathrm{I}}{m}\right) \frac{d x}{x} \\
\times\left(\sigma_{r_{0}}-\sigma_{t_{0}}\right) \\
=(\mathrm{I} 7) \cdot(85)\end{array}$ & $\begin{array}{c}(87) \\
(27) \cdot \psi \\
(27) \cdot(102)\end{array}$ & $\begin{array}{c}(88) \\
\frac{d \sigma_{r_{0}}}{m} \\
0,2 \cdot(83)\end{array}$ & $\begin{array}{c}(89) \\
a \sigma_{t_{0}}= \\
+(86) \\
-(87) \\
+(88)\end{array}$ & $\begin{array}{c}(90) \\
\sigma_{t_{02}}= \\
\sigma_{t_{01}}+d \sigma_{t_{0}}\end{array}$ \\
\hline 10 & & & & & & & & & & \\
\hline I I 1 & $\begin{array}{l}0,1 \\
\ldots\end{array}$ & 0,100 & $\begin{array}{c}-0,129 \\
\ldots\end{array}$ & & & $\begin{array}{c}0,12 \\
\ldots\end{array}$ & $\begin{array}{l}6,30 \cdot 10^{3} \\
\ldots \ldots\end{array}$ & & & \\
\hline 12,1 & $\begin{array}{c}0,0915 \\
. .\end{array}$ & $\begin{array}{l}0,091 \\
\cdots \cdots\end{array}$ & $\begin{array}{l}-0, I 4 I \\
. . .\end{array}$ & & & $\begin{array}{c}0,109 \\
\ldots .\end{array}$ & $\mid \begin{array}{c}6,30 \cdot 10^{3} \\
\ldots \ldots\end{array}$ & & & \\
\hline I 31 & $\begin{array}{l}0,084 \mathrm{I} \\
\ldots\end{array}$ & $\begin{array}{c}0,0835 \\
\ldots\end{array}$ & $\begin{array}{l}-0,154 \\
. . .\end{array}$ & & & $\begin{array}{c}0, \mathrm{t} 00 \\
\ldots .\end{array}$ & $\begin{array}{l}7,15 \cdot 10^{3} \\
. \ldots\end{array}$ & & & \\
\hline 141 & & & & 1 & & & & & & \\
\hline
\end{tabular}

Zahlentafel 6 .

\begin{tabular}{|c|c|c|c|c|c|c|c|c|c|c|}
\hline$d x$ & $\begin{array}{c}(92) \\
(32) \sigma_{r_{0}} \\
(32) \cdot(84)_{1}\end{array}$ & $\begin{array}{c}(93) \\
\left(3^{6)} \sigma_{t_{0}}\right. \\
(36) \cdot(90)_{1}\end{array}$ & $\begin{array}{c}\text { (94) } \\
(42)\left(\frac{d \psi}{d x}\right) \\
(42) \cdot(100)\end{array}$ & $\begin{array}{c}(95) \\
(49) \cdot \psi_{1} \\
(49) \cdot(I 02)\end{array}$ & $\begin{array}{c}(96) \\
(92)+(93) \\
-(94)+(95)\end{array}$ & $\begin{array}{c}(97) \\
(96)-(51)\end{array}$ & $\begin{array}{c}(98) \\
(97):(40) \\
=\frac{d^{2} \psi}{d x^{2}}\end{array}$ & $\begin{array}{c}\text { (99) } \\
\frac{d^{2} \psi}{d x^{2}}(d x)\end{array}$ & $\left\{\begin{array}{c}(100) \\
\left(\frac{d y}{d x}\right) \\
(100)_{2}= \\
(100)_{1}+(99)\end{array}\right.$ & $\begin{array}{c}\text { (IOI) } \\
d \psi= \\
\left(\frac{d \psi}{d x}\right) d x \\
(100) \cdot(2)\end{array}$ \\
\hline 10 & & & & & & & & & & \\
\hline $\begin{array}{lll} & 1 & 1 \\
\end{array}$ & $\begin{array}{c}0,271 \cdot 10^{-3} \\
\therefore . .\end{array}$ & $\begin{array}{c}0,0136 \cdot 10^{-3} \\
. .\end{array}$ & $\begin{array}{l}+ \text { IOI } 5 \\
. . .\end{array}$ & $\begin{array}{c}108, I \\
\ldots .\end{array}$ & & $\begin{array}{c}+70,7 \cdot 10^{-3} \\
. \cdots\end{array}$ & $\begin{array}{c}: 10,7 \cdot 10+3 \\
. . .\end{array}$ & & & \\
\hline 12 & $\begin{array}{c}0,357 \cdot 10^{-3} \\
\ldots . . .\end{array}$ & $\begin{array}{c}0,0149 \cdot 10^{-3} \\
\cdots\end{array}$ & $\begin{array}{l}+1066 \\
. . . \\
\end{array}$ & $\begin{array}{r}98,4 \\
\ldots . . . \\
\end{array}$ & & $\mid \begin{array}{c}+85 \cdot 10^{-3} \\
. \ldots\end{array}$ & $\begin{array}{l}: 11,8 \cdot 10+3 \\
. . .\end{array}$ & & & - \\
\hline 13 & $\begin{array}{c}0,422 \cdot 10^{-3} \\
\ldots . .\end{array}$ & $\begin{array}{c}0,016 \cdot \mathrm{IO}^{-3} \\
\ldots\end{array}$ & $\begin{array}{l}+1080 \\
. . . .\end{array}$ & $\begin{array}{c}90,4 \\
. \quad \ldots .\end{array}$ & & $\begin{array}{c}+100 \cdot 10^{-3} \\
\ldots . .\end{array}$ & $\begin{array}{l}: 12,9 \cdot 10+3 \\
. \quad . .\end{array}$ & & & \\
\hline I4 I & & & & & & & & & & \\
\hline
\end{tabular}

Zahlentafel 7 .

\begin{tabular}{|c|c|c|c|c|c|c|c|c|c|}
\hline $\begin{array}{l}\text { (I) } \\
x \\
d x\end{array}$ & $\begin{array}{c}(\mathrm{IO2}) \\
\psi_{2}= \\
\psi_{1}+d \psi \\
(\mathrm{IO2})_{1}+(\mathrm{IOI})_{2}\end{array}$ & $\begin{array}{c}(103) \\
m\left(\frac{d \psi}{d x}\right) \\
3,33 \cdot(100)\end{array}$ & $\begin{array}{c}(104) \\
\frac{\psi}{x} \\
(102):(1)\end{array}$ & $\begin{array}{c}\text { (105) } \\
m \frac{d \psi^{\prime}}{d x}+\frac{\psi}{x} \\
(1 \circ 3)+(104)\end{array}$ & $m \frac{\psi}{x}$ & $\frac{d \psi}{d x}+m \frac{\psi}{x}$ & $\begin{array}{c}(108) \\
c \frac{h}{2} \cos \varphi \times \\
\left(m \frac{d \psi}{d x}+\frac{\psi}{x}\right) \\
(54) \cdot(105)\end{array}$ & $\begin{array}{c}(1 \circ 9) \\
c \frac{h}{2} \cos q \times \\
\left(\frac{d \psi}{d x}+m \frac{\psi}{x}\right) \\
(54) \cdot(107)\end{array}$ & $\begin{array}{r}h d s \sigma_{t, 0} \\
(53) \cdot(90)\end{array}$ \\
\hline 10 & & & & & & & $\begin{array}{l}0,562 \cdot 10^{6} \\
. . . \\
\end{array}$ & & $\begin{array}{r}6,02 \\
. \quad . \\
\end{array}$ \\
\hline II & & & & & & & $\begin{array}{l}0,562 \cdot 10^{6} \\
. . .\end{array}$ & & $\begin{array}{r}6,03 \\
. \\
\end{array}$ \\
\hline I2 1 & & & & & & & $\begin{array}{l}0,562 \cdot 10^{6} \\
.\end{array}$ & & $\begin{array}{r}6,03 \\
. \quad . \\
\end{array}$ \\
\hline 13.1 & & & & & & & $\begin{array}{l}0,562 \cdot 10^{6} \\
. . . \\
\end{array}$ & & $\begin{array}{r}6,03 \\
. \quad .\end{array}$ \\
\hline I 41 & & & & & & & & & \\
\hline
\end{tabular}


Die Ergebnisse dieser beiden Hauptdurchrechunngsgruppen sind in die l'ig. I 5 bis 20 eingetragen und geben ein ebenso lehrreiches wie anschauliches Bild über den Verlauf der Normalspannungen in den einzelnen Punkten der Platte und die Wanderung der einzelnen Punkte der Meridian-Mittelfaser infolge der Durchbiegung. Wir heben besonders hervor, daß die einzelnen Ergebnisse wie folgt zusammengestellt sind:
Für Fall . . . . . . . . . . . .
Zahlen beispiel
3
4

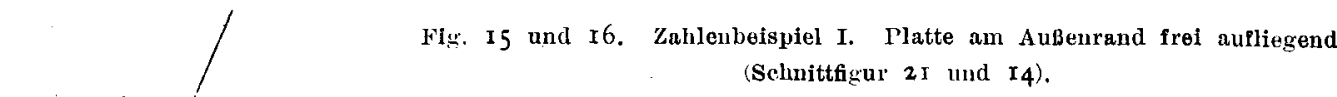

Fig. I 5. Radialspannung $\sigma_{r}$ in $\mathrm{kg} / \mathrm{qem}$
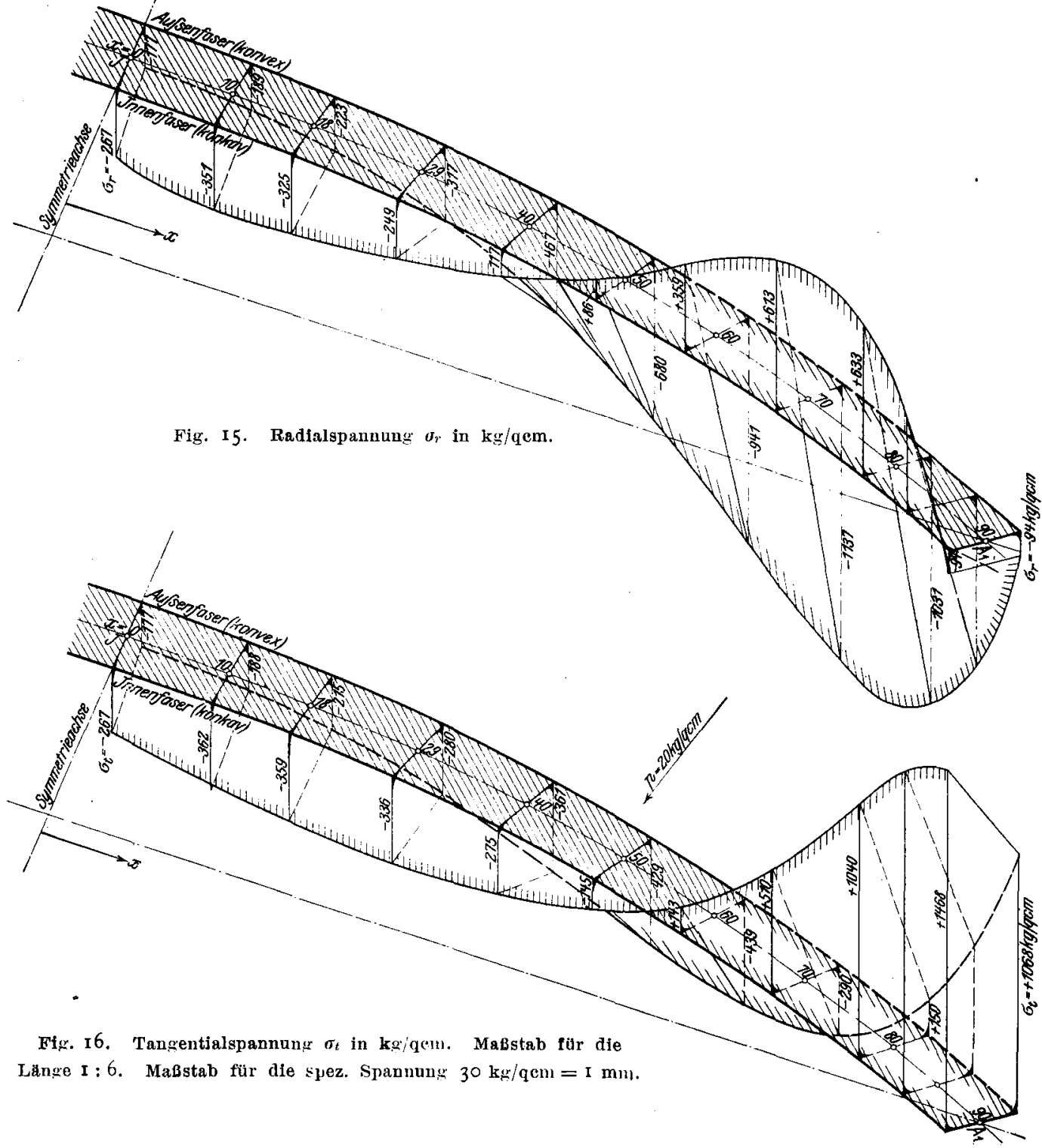
Platte am Rand . . . . . . frei aufliegend, eingespannt (aber in Richtung des Halbmessers beweglich.)

Axonometrisches Bild der »Radialspannungen« Fig. I 5 " » »'Tangentialspannungen « " I6 Fig: 17 Durchbiegung bezogen auf die Plattenmitte I 8

Fig. I9

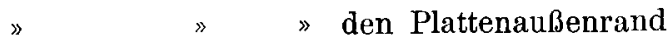
$\gg 20$.

Die zeichnerischen Maßstäbe sind in die einzelnen Figuren eingetragen.

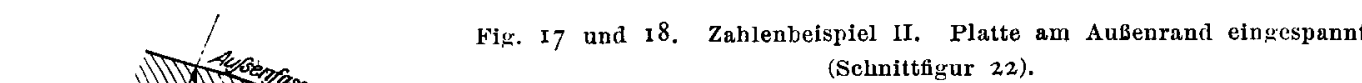
(Schnittfigur 22).

Fig. I 7. Radialspannung $o_{r}$ in $\mathrm{k} \underline{g}_{j} \mathrm{q} \mathrm{cm}$.
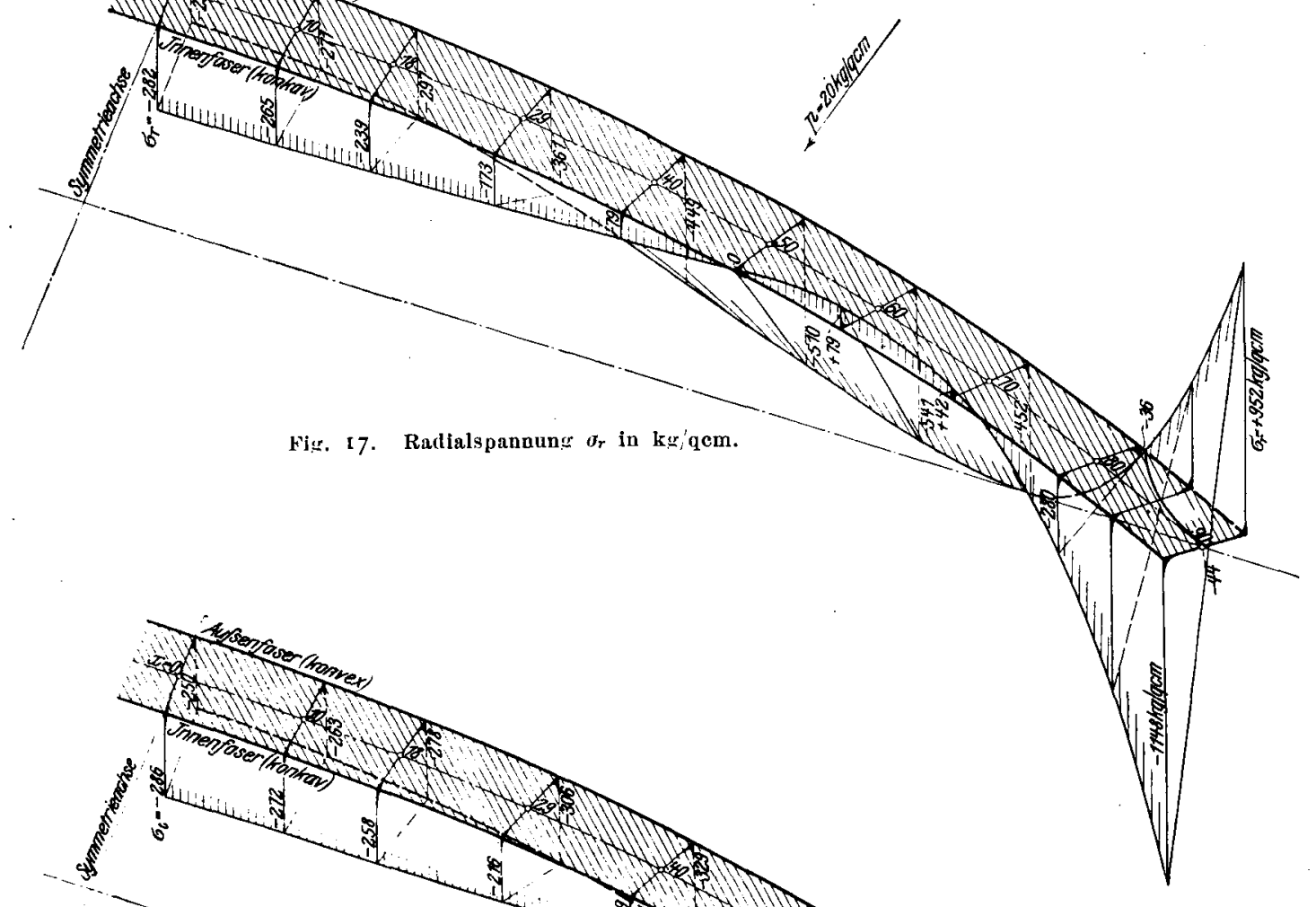

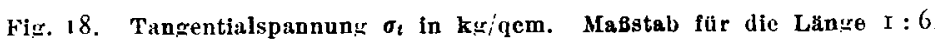
Ma@stab für die spez. Spannung $30 \mathrm{~kg} / \mathrm{qcm}=\mathrm{I} u \mathrm{~m}$. 

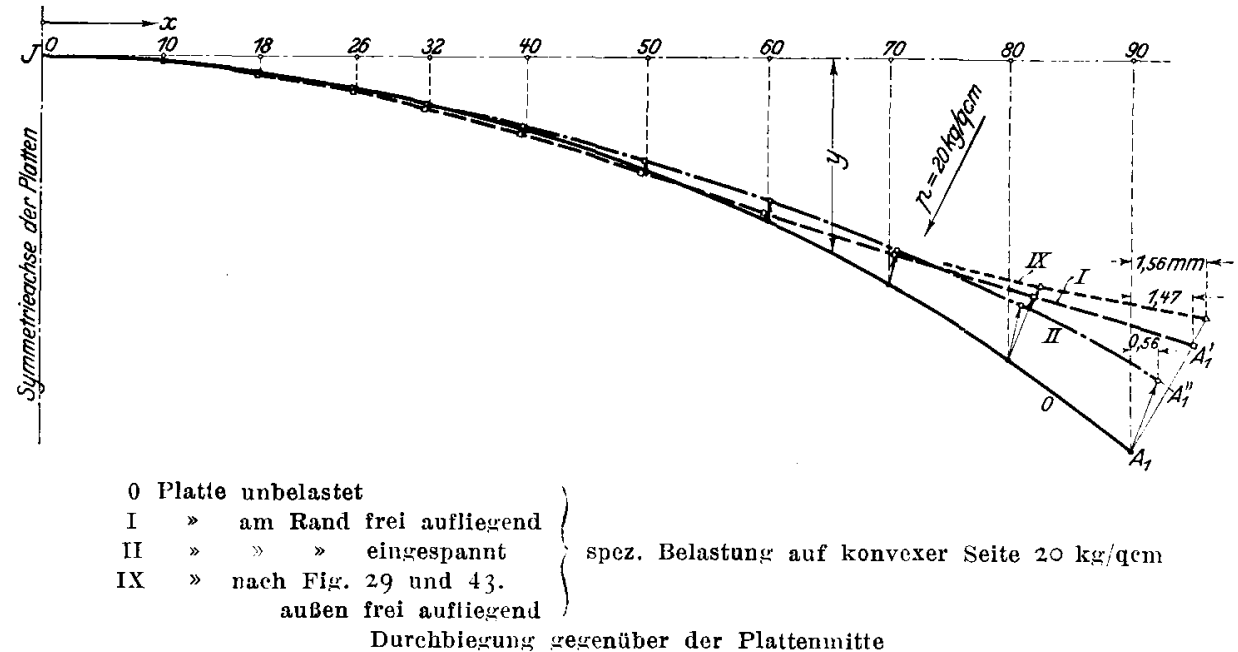

Maßstab fül die Mittelfaser I: 8. Maßstab für die Ausbiegung $5:$ I. Verhältnis der gezeichneten Ausbiegung zur aufozezelcheten Mittelfaser 40: I.

Fig. 19. Lage der Mitlelfaser des Meridianschnittes.

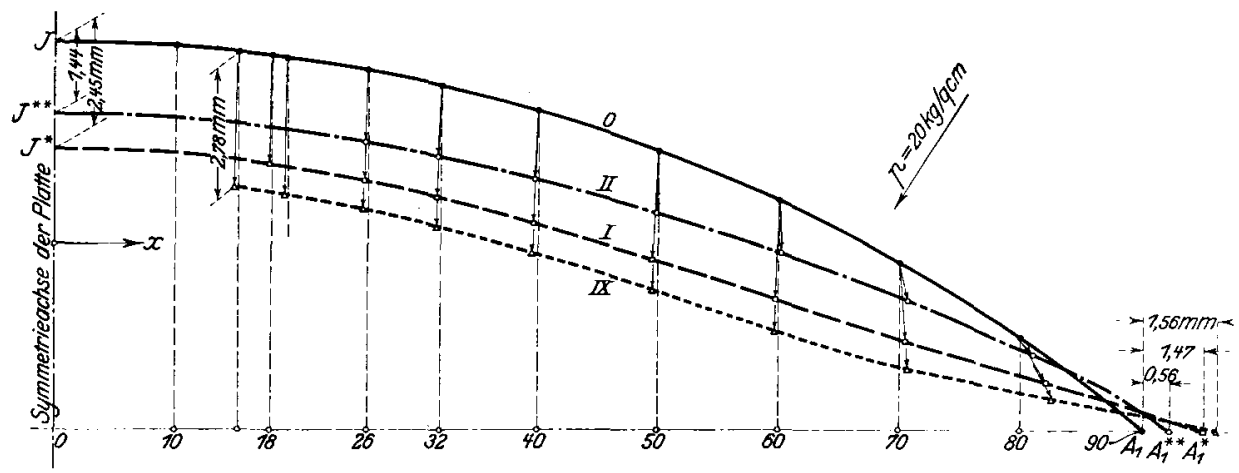

Fir. 20. Durchbiegung gegenaber der Auflagefläche der Platte. Maßstabe wie in Fig. I9.

\section{Diskussion der Rechnungsergebnisse der Zahlenbeisplele I und II.}

Ein Vergleich der Figuren I $_{5}$ bis I 8 zeigt, daß die Normalspannungen in der am Rand eingespannten Platte durchschnittlich viel niedriger sind, als in der frei aufliegenden Platte. In letzterer tritt die größte Spannung als Tangentialspannung $\sigma_{t}=+\mathrm{I} 468 \mathrm{~kg} / \mathrm{qcm}$ (Zug) an der Innenfaser im Abstand $x=90 \mathrm{~cm}$, Fig. 16 , die nächst größte als Radialspannung $\sigma_{r} \sim-1180 \mathrm{~kg} / \mathrm{qcm}$ (Druck) an der Außenfaser zwischen den berechneten Punkten $x=70$ und $80 \mathrm{~cm}$ auf, Fig. I 5. Bei der am Rand eingespannten Stelle beträgt die Höchstbeanspruchung - I 48 $\mathrm{kg} / \mathrm{qcm}$. Es ist dies die Druckspannung in Richtung des Meridians an der Innenfaser des Außenrandes, Fig. I7. Die höchste an dem am Rand eingespannten Deckel auftretende Tangentialspannung finden wir ebenfalls am außeren Rand, und zwar an der äußeren Faser mit $+726 \mathrm{~kg} / \mathrm{qcm}(\mathrm{Zug})$. Alle anderen am eingespannten Deckel erscheinenden Spannungen sind viel kleiner als die soeben genannten.

Die Figuren 15 bis 18 zeigen ferner, daß tür beide Lagerungsarten des Deckels Punkte zu finden sind, die entweder in Richtung des Meridians oder des Parallelkreises spannungslos sind. Es ist dies der Fall z. B. laut Fig. 15 in Rich- 
tung des Meridians an der inneren Begrenzungsfaser im Abstand $x=45 \mathrm{~cm}$ von der Symmetrieachse, laut Fig. I6 in »tangentialer" Richtung an der äußeren Begrenzungsfaser bei $x=78 \mathrm{~cm}$, an der inneren Begrenzungsfaser bei $x=$ $56 \mathrm{~cm}$ usw.

Eine weitere Betrachtung der die "Radialspannungen " am frei aufliegenden und am außen eingespannten Deckel darstellenden Fig. I5 und I7 zeigt, daß in beiden Fällen zwischen der Symmetrieachse und dem Außenrand je ein Querschnitt vorkommt, der keiner Biegungs-, sondern nur einfacher normaler Druckspannung unterworfen ist. Es ist dies jeweils derjenige Kegelschnitt, in welchem im graphischen Bild die Radialspannungen in der inneren und äußeren Begrenzungsfaser gleich groß erseheinen. Schätzungsweise trifft dies zu in Fig. I 5 für die Kote $x=22 \mathrm{~cm}$, in Fig. I 7 für den Halbmesser $x=8 \mathrm{~cm}^{1}$ ). In Fig. I 5 verschwindet das Biegungsmoment natürlich ein zweites Mal für $x=90 \mathrm{~cm}$ laut Voraussetzung, daß die Platte dort frei aufliege.

Die in den Fig. I 5 bis 18 gegebenen Darstellungen der Spannungsverteilungen lehren ferner, daß die Platte einem "Körper gleicher Festigkeit" angeniihert werden könnte, wenn sie in der Mitte dünner, gegen außen und besonders am Rand dicker gehalten wäre.

\section{Durchbiegung des nach Fig. 14 ausgebildeten und auf der konvexen Seite mit $20 \mathrm{~kg} / \mathrm{qcm}$ belasteten Deckels.}

In den Fig. I9 und 20 ist die rechnerische Durchbiegung der Mittelfaser des Deckels dargestellt für die zwei Fälle:

I) Die Platte ist am Rand frei aufliegend (Kurve I).

II) Die Platte ist am Rand so eingespannt, daß ihr Außenquerschnitt sich wohl in Richtung senkrecht zur Symmetrieachse parallel verschieben, nicht aber verdrehen kann. Der Kürze halber wollen wir diesen Fall mit »Platte außen eingespannt« bezeichnen, obschon dieses Einspannen nur bedingt geschieht (Wahrung der Verschiebbarkeit) (Kurve $I I$ ).

Die Kurve $O$ stellt die Meridian-Mittelfaser (Kreisbogen) im unbelasteten Zustand dar. Es ist wohl zu benchten, daß die Maßstäbe für die Mittelfaser einerseits und für jhre Durchbiegung anderseits verschieden sind, und zwar ist die Meridian-Mitteliaser aufgezeichnet im Maßstab $\mathrm{I}: 8$, die Aenderung von deren Koordinaten im Maßstab $5: 1$, so daß also die Aenderungen der Koordinaten relativ zu den Koordinaten selbst in fofacher Vergrößerung dargestellt sind.

Weil wir von der Mitte aus gerechnet haben, ist in Fig. I9 demgemäß die Verschiebung der einzelnen Punkte relativ zum Mittelpunkt $J$ aufgetragen und dabei die Annahme gemacht, daß alle weiter außen liegenden Punkte sich verschieben können. Die Mitteliaser geht ans ihrer ursprïnglichen Lage $J A_{1}$ (Deckel unbelastet) uiber entweder in die Lage $J A_{1}^{\prime}$ oder in die Lage $J A_{1}^{\prime \prime}$, je nachdem der Deckel am Rand »frei aufliegend « oder »eingespannt" ist.

Die Darstellung Fig. 20 ist für die der Wirklichkeit eher entsprechende Annahme getroffen, dals der Randpunkt $A_{1}$ sich nur auf der Wagerechten, d. i. senkrecht zur Srmmetrieachse, nicht abel parallel hierzu bewegen könne, und zwar nach den Lagen $A_{1}{ }^{*}$ oder $\boldsymbol{A}_{1}{ }^{* *}$, je nachdem die Platte am Rand frei aufliegt oder eingespannt ist. Der Mittelpunkt der Platte bewegt sich hierbei aus der ursprünglichen Lage $J$ nach $J^{*}$ oder $J^{* *}$.

1) Hier zeigt die elastische Linie relative Inflexjonspunkte. 


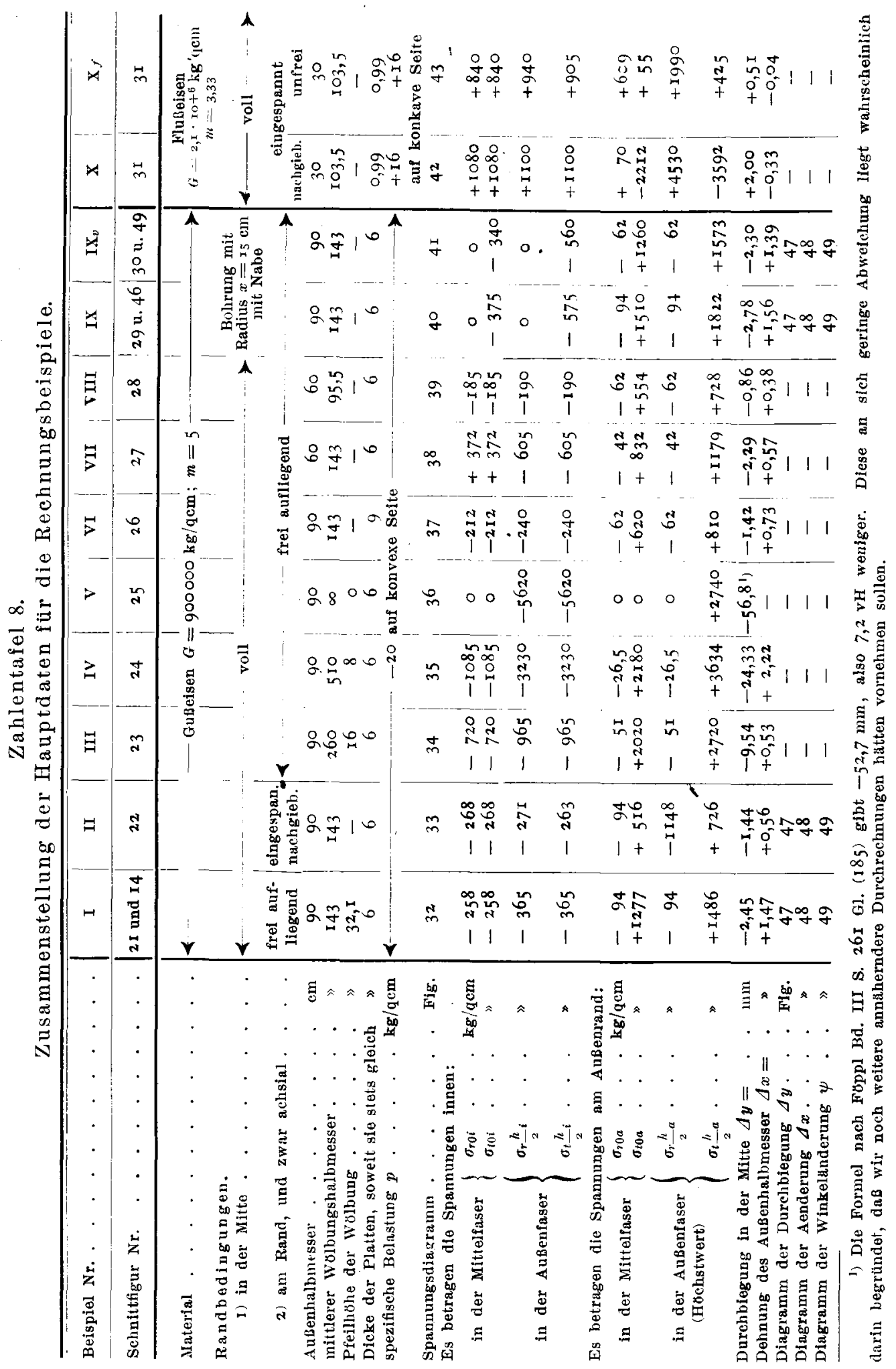


Fir. $2 \mathrm{I}$ bis $3 \mathrm{I}$. Mabstab für Fig. $2 \mathrm{I}$ bis 30 I $: 45$.

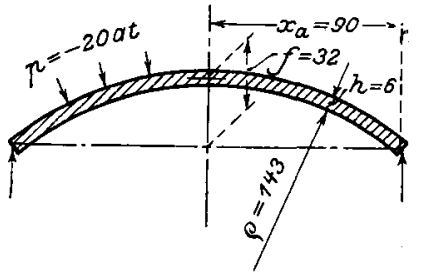

Fig. 2I. I.

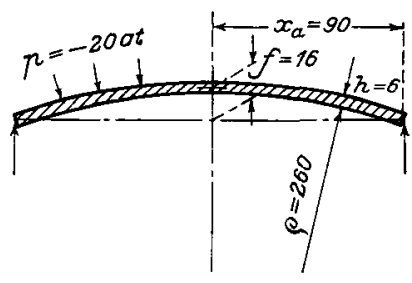

Firig. 23. III.

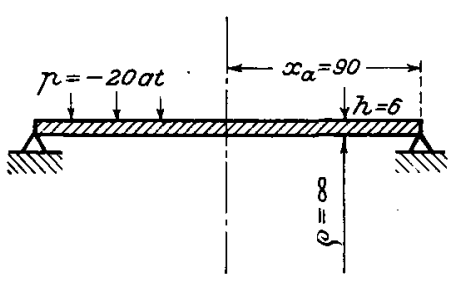

Fix. 25. V.

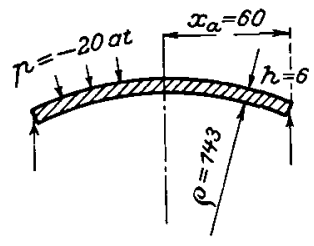

Fig. 27. VII.

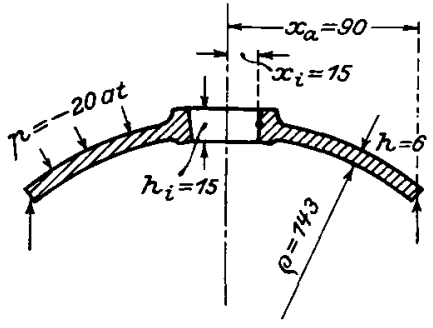

Fig. 29. IX.

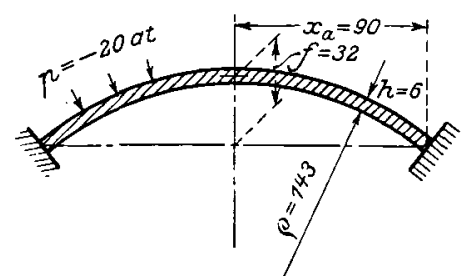

Fig. 22. II.

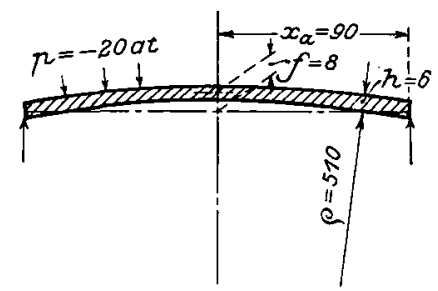

Fig. 24. IV.

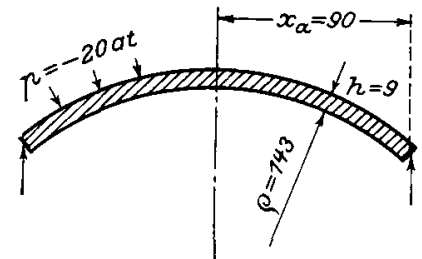

Hiw. 26. VI.

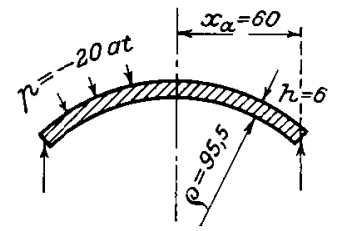

Fig. 28. VILI.

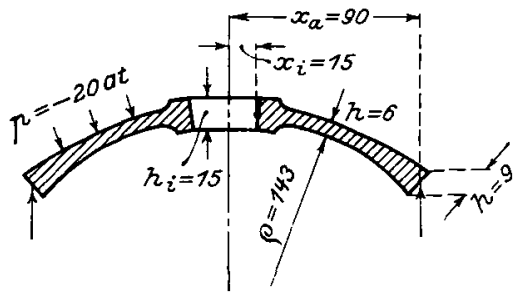

Fig. 30. IX .

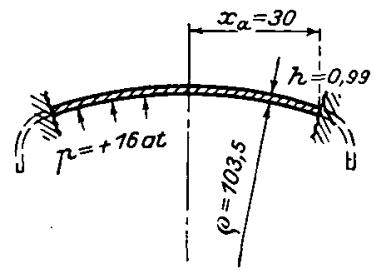

Fír. 3I. X. Masstab $2: 45$. 

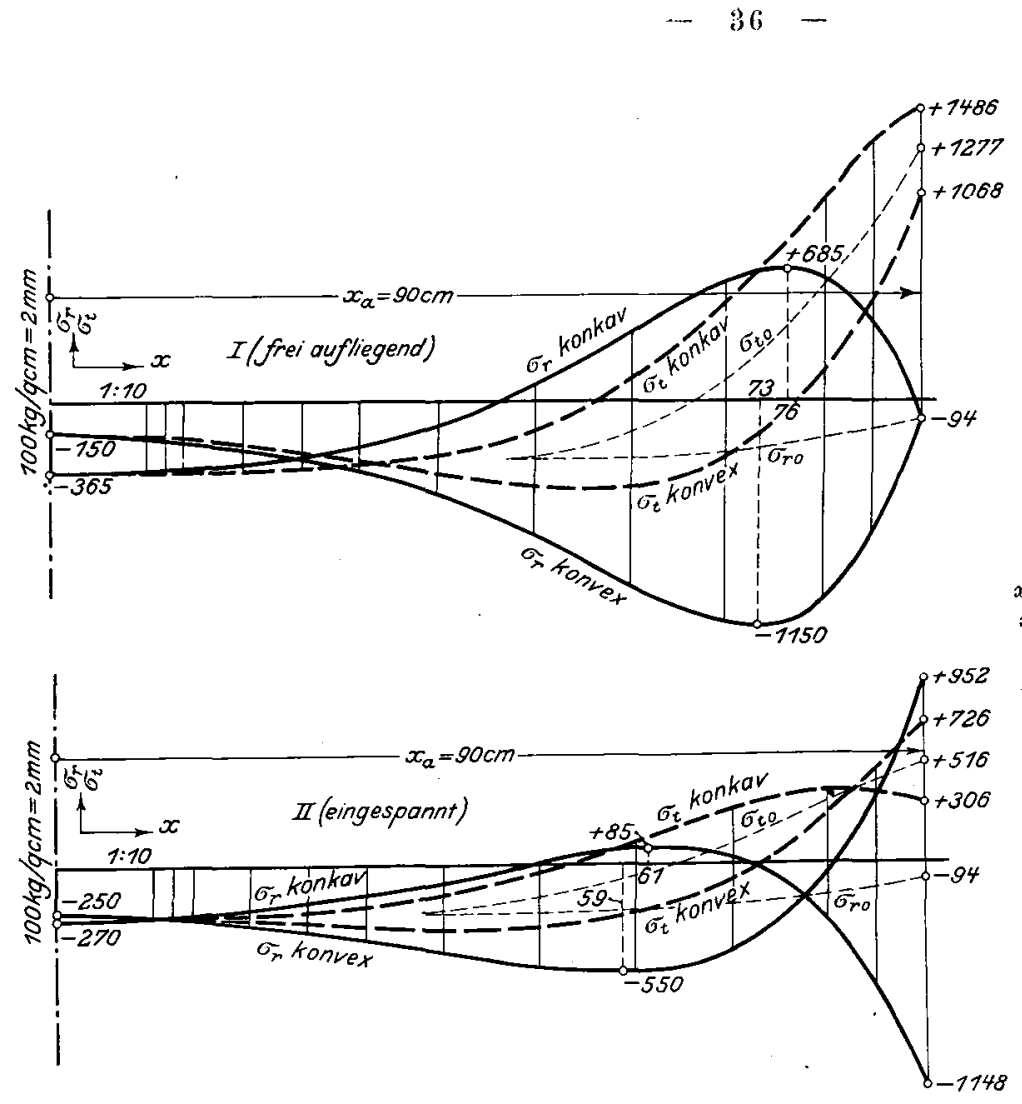

Fig. 32 und 33 .

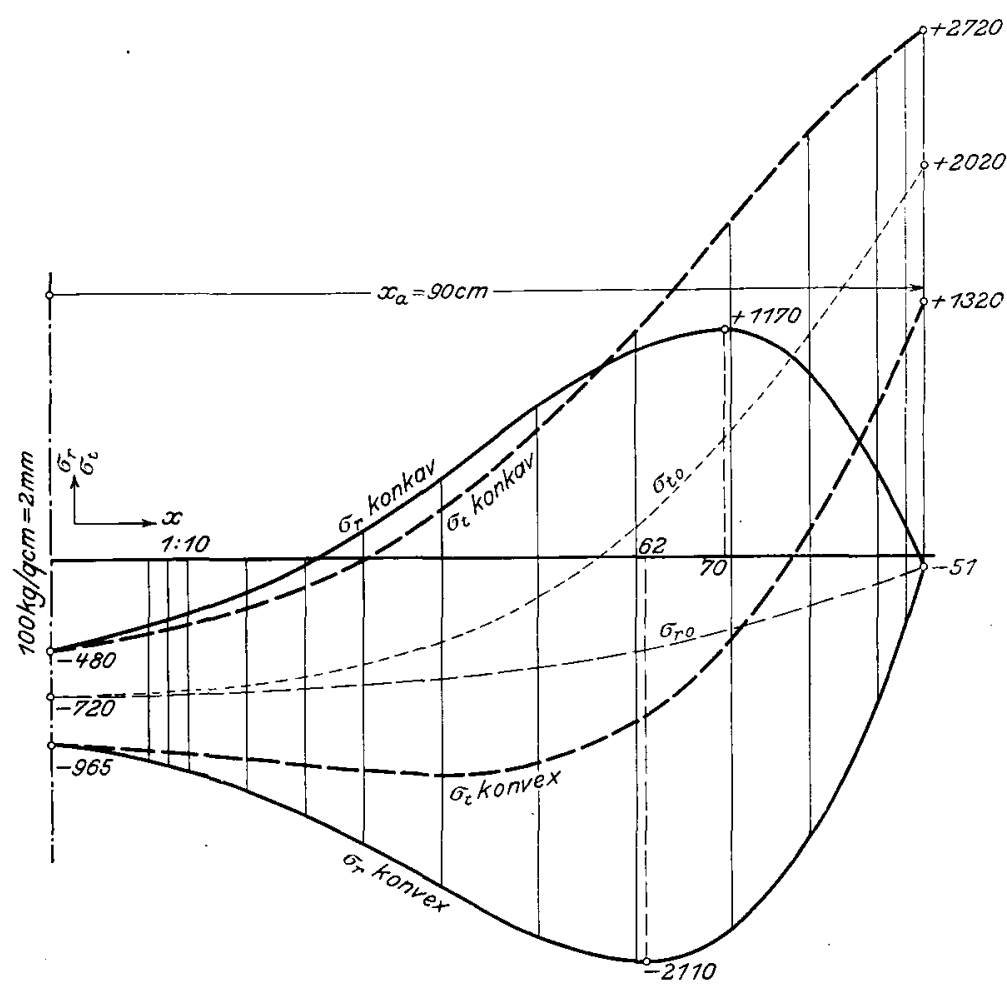

Fi $x .34$.

\begin{tabular}{|c|c|c|}
\hline Zahlenbeispiel & $\begin{array}{l}\quad I \\
\text { frei auf- } \\
\text { : liegend }\end{array}$ & $\begin{array}{c}\text { II } \\
\text { einge- } \\
\text { spannt }\end{array}$ \\
\hline$\rho=$ in $\mathrm{cm}=$ konst & 143 & I 43 \\
\hline$x_{a}=\mathrm{i}$ & 90 & 90 \\
\hline$x_{i}=$ in $\mathrm{cm}$ & 0 & \\
\hline$=$ in $\mathrm{cm}=$ konst & 6 & 6 \\
\hline$=$ in $a t=$ & -20 & -20 \\
\hline
\end{tabular}

Material: Gußeisen.
Zahlenbeispiel III.

$g=260 \mathrm{~cm}=$ konst

$x_{a}=90 \mathrm{~cm}$

$x_{i}=0$

$h=6 \mathrm{~cm}=\mathrm{konst}$

$y=-20$ at

Material: GuBeisen. 
Die für die wirklichen Koordinatenänderungen gefundenen Rechnungswerte sind in den Fig. I9 und 20 eingeschrieben. (Für Fall II, d. h. "außen eingespannt, ist der Wert $J_{x_{a}}$ nicht zuverlaßlich. Vermutlich liegt ein Rechenfehler vor infolge der Wahl zu großer Intervalle für $d x$ gegen den Außenrand hin.)

Nach unserer Rechnung und nach Fig. 20 kommt die größte Aenderung der Koordinate in Richtung parallel zur Symmetrieachse in der Mitte der

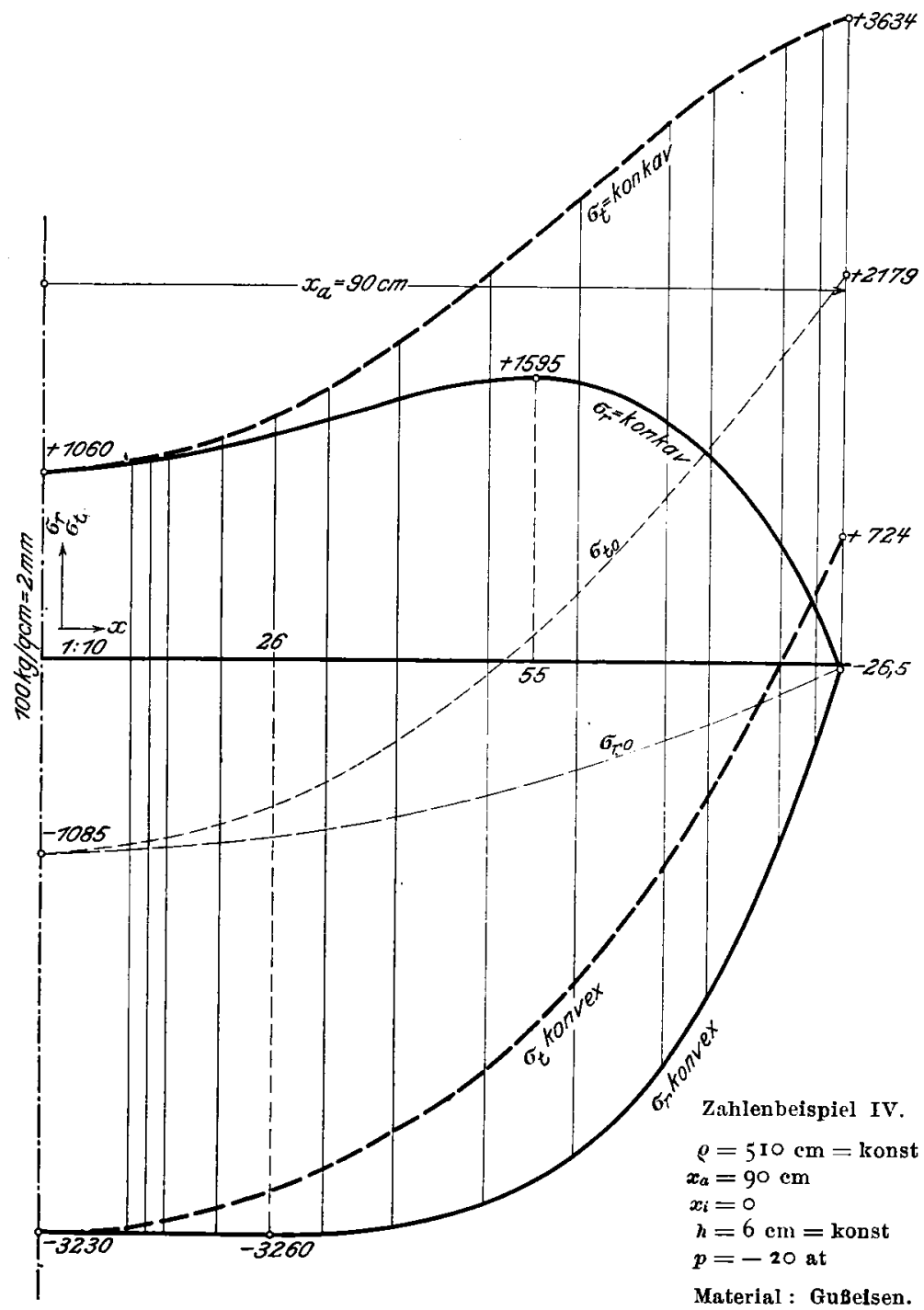

Fig. 35 .

Platte vor, wie nicht anders zu erwarten war. Sie beträgt bei der frei aứliegenden Platte 2,45 mm, bei der am Rand eingespannten Platte nur I,44 mm.

Der äußerste Punkt $\boldsymbol{A}_{1}$ der Meridian-Mittelfaser verschiebt sich um 1,47 mm nach außen, wenn die Platte außen frei aufliegend, dagegen nur um rd. $0,56 \mathrm{~mm}$, wenn die Platten »außen eingespannt.

Von der Wiedergabe aller Rechnungs- und insbesondere der Zwischenwerte wurde mit Rücksicht auf deren große Zahl abgesehen. 
Im AnschluB an diese eingehend besprochenen Beispiele I und if wurden noch zelın weitere Beispiele durchgerechnet. In Zahlentalel 8 sind von allen zwöll Beispielen die ihnen zugrunde gelegten Berlingungen zusammengestellt. Die Hauptabmessungen der durehgerechneten Platten finden sich in den Fig. 2I bis 3I. Diese Beispiele geben über folgende Fragen Aufsehluh:

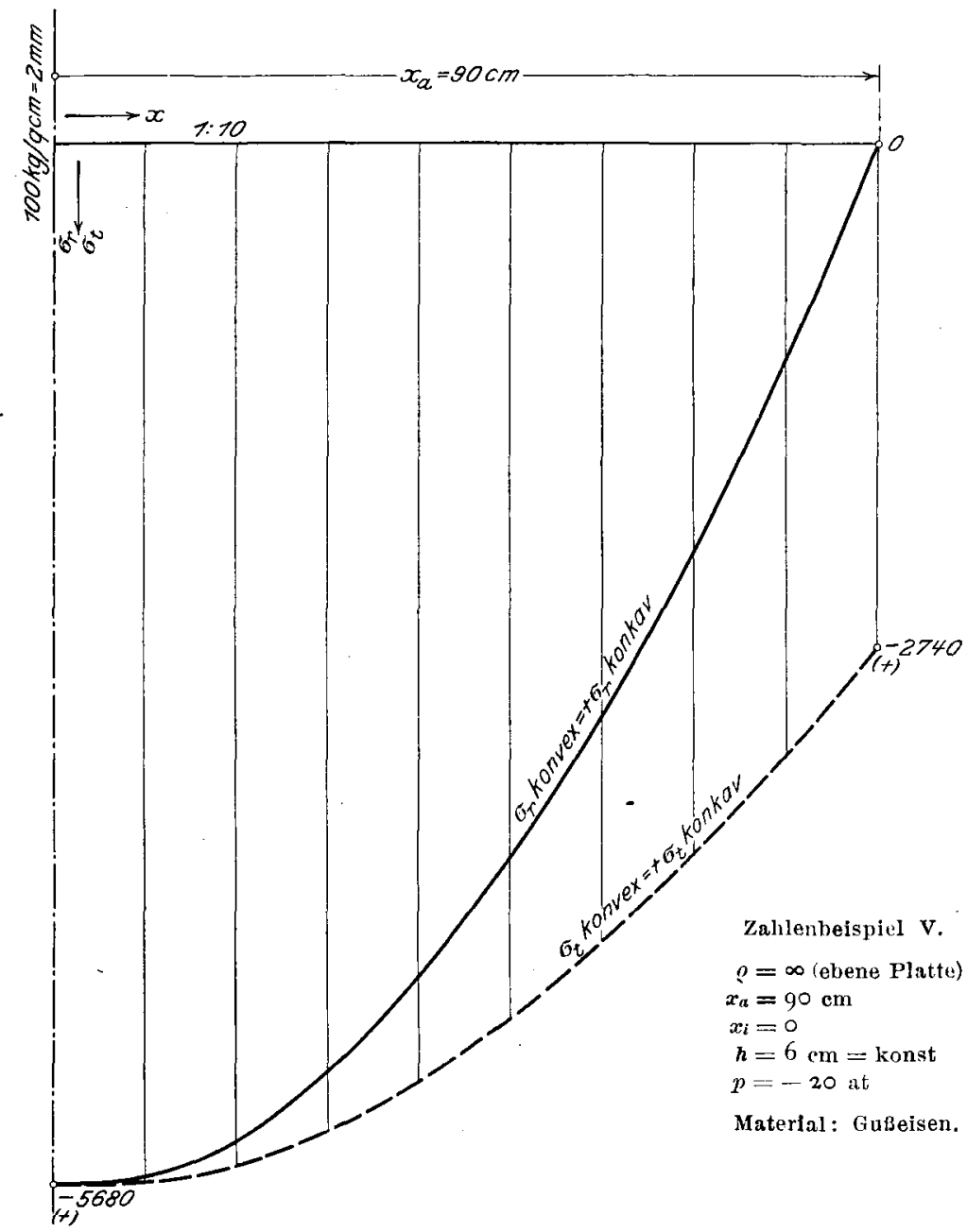

Fig. 36 .

a) Unterschied zwischen den Randbedingungen "Platte außen frei aufliegend " und "eingespannt".

b) Einfluß der Plattenwölbung.

c) " Plattendicke.

d) " "Größe des äußeren Halbmessers " $x_{n}$ ".

e) $"$ Bohrung und der versteifenden Nabe.

f) " "Verdickung des Plattenrandes.

g) Unterschied zwischen »außen nachgiebig eingespannt« und * fest eingespannt«.

Die einer und derselben Gruppe a) bis g) angehörigen Platten unterseheiden sich nur durch die für den jewciligen Programmpunkt gekennzeichneten Daten. 
Die Beispiele I bis $\mathrm{IX}_{v}$ haben das gemein, daß es sich um die Berechnungen gubeiserner Platten handelt $(E=900000 \mathrm{~kg} / \mathrm{qcm} ; m=5)$, welche von der konvexen Seite mit $p=20 \mathrm{~kg} / \mathrm{qcm}$ belastet sein sollen. Diese Belastung gibt zugestandenermaßen für fast alle Beispiele für Gußeisen durchaus unzulässig

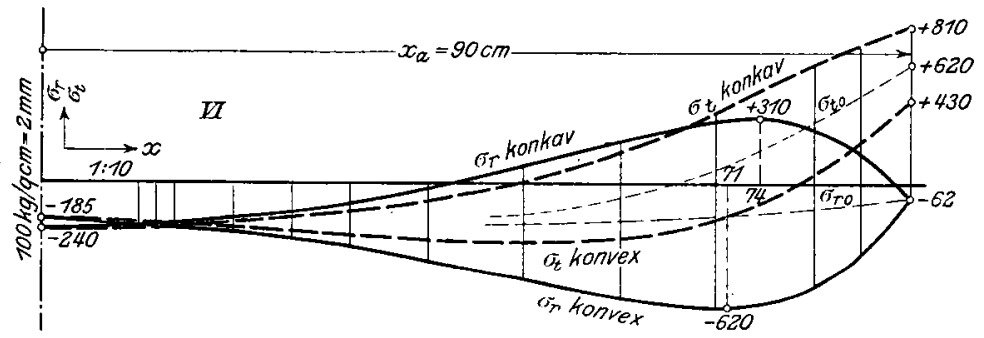

Fi $\underline{x} .37$.

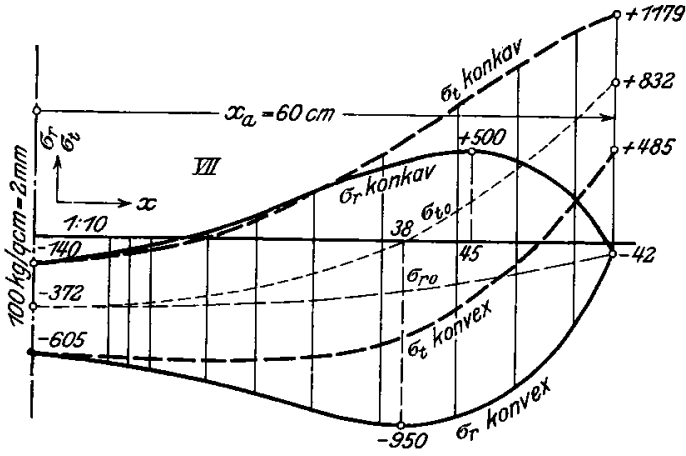

\begin{tabular}{l|c|c:c} 
Zahlenbeispiel & VI & VII : VIII
\end{tabular} \begin{tabular}{ll|l|l|l}
$\varphi=-$ konst & em & I 43 & I 43 & 95.5
\end{tabular} \begin{tabular}{ll|l|l:l}
$x_{\boldsymbol{a}}=-$ & $\$$ & 90 & 60 & 60
\end{tabular} $x_{i}=! \quad 》 \quad 0000$ \begin{tabular}{lc|r|r:r}
$h=$ konst & $\gg$ & 6 & 6 & 6
\end{tabular}

Fig. 38.

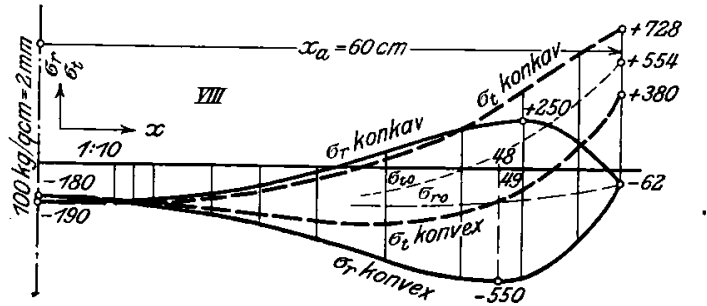

Fig. 39.

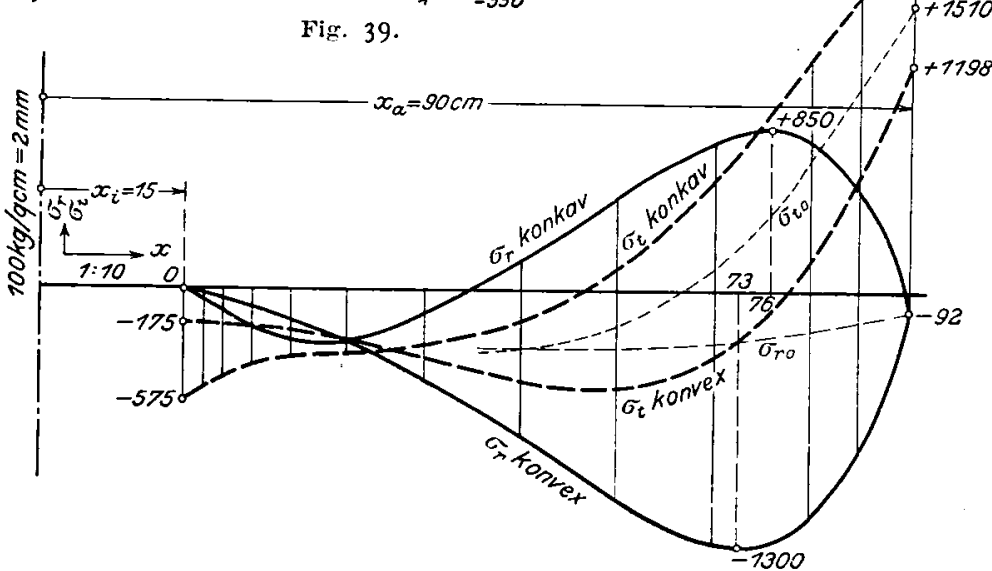

Zablenbeispiel IX. $\rho=143 \mathrm{~cm}=$ konst, $x_{a}=90 \mathrm{~cm}, x_{i}=15 \mathrm{~cm}$ der Bohrung in der Mitte, $h=6 \mathrm{~cm}=$ konst von $x=32$ bis $x_{a}=90 \mathrm{~cm}, \quad p=-20$ at, Material: Gubeisen. 


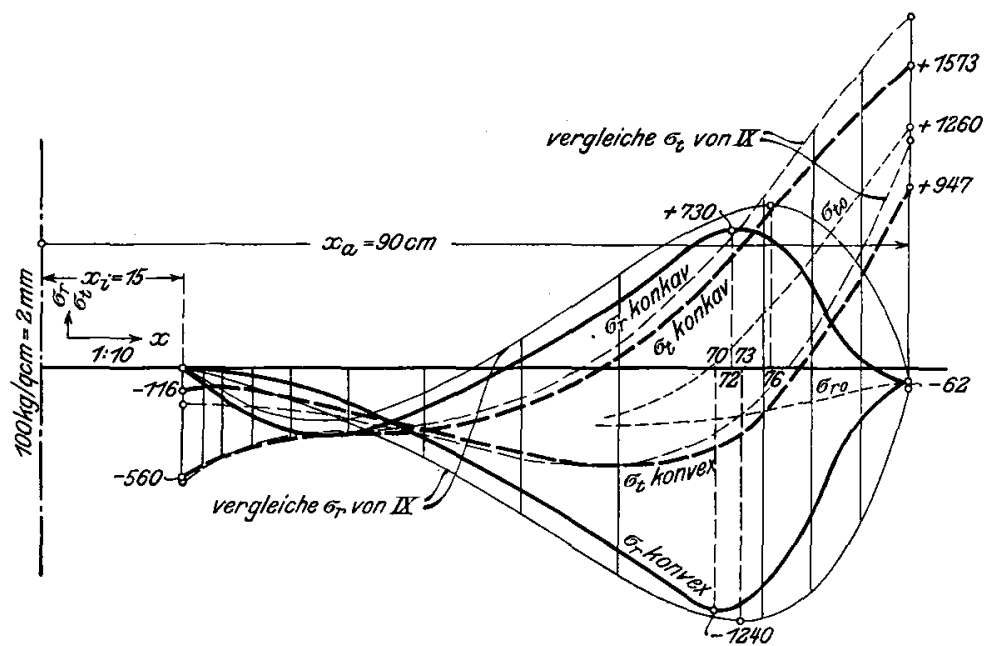

Zahlenbeispiel $\mathrm{IX}_{v} . \rho=\mathrm{I} 43 \mathrm{~cm}=$ konst, $x_{a}=90 \mathrm{~cm}, x_{i}=15 \mathrm{~cm}$ der Bohrung in der Mitte, $h=$ koust, $\quad p=-20$ at, Material: Gubeisen.

Fig. 4I.

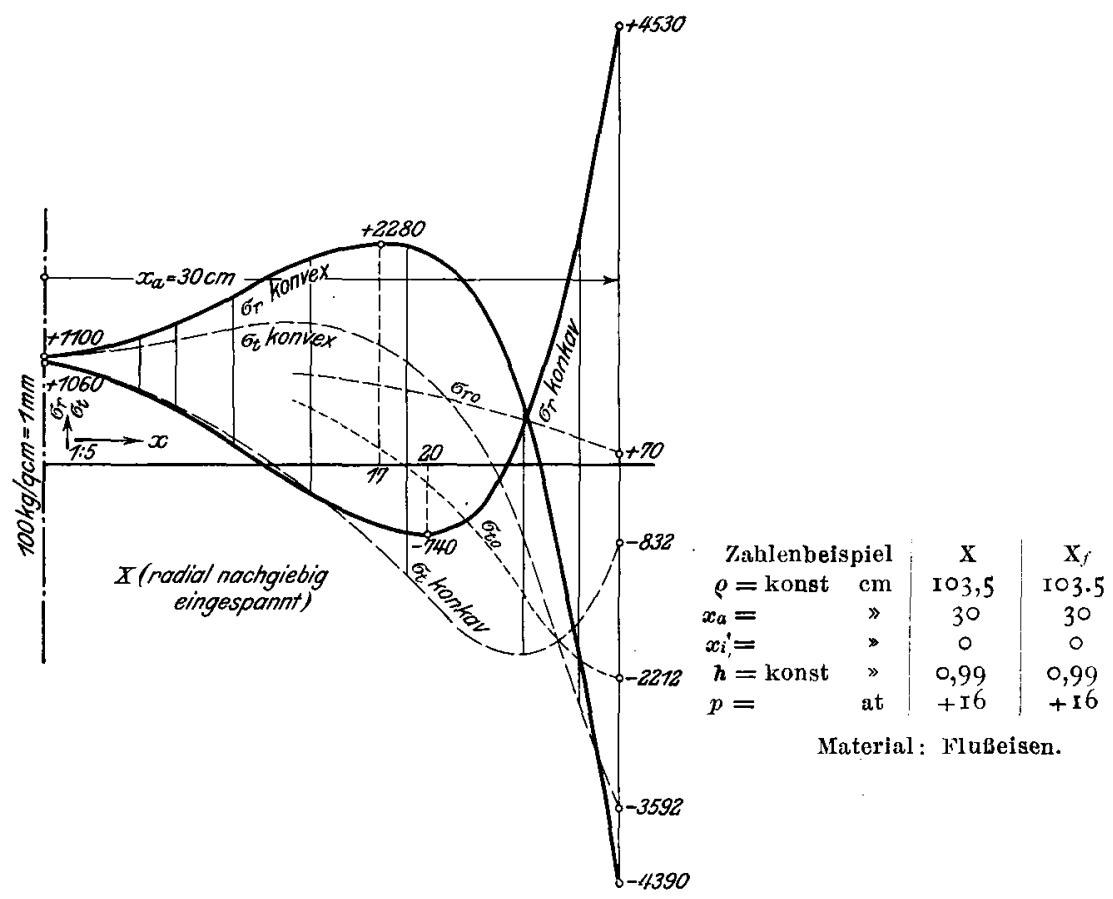

Fig, 42.

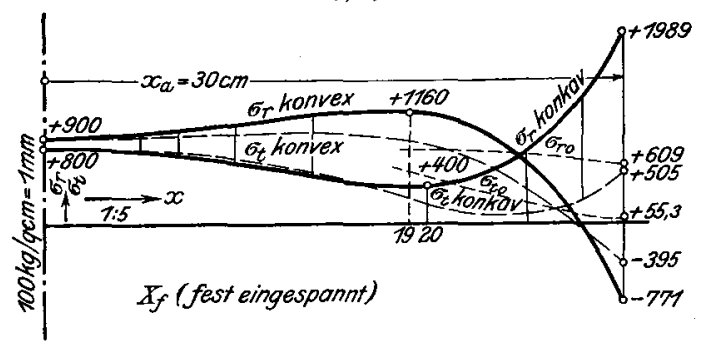

Fig. 43 . 
hohe Beanspruchungen. Sie soll deshalb auch nur für unsere vergleichsweise Rechnung, nicht aber in Wirklichkeit Gültigkeit haben. Wir erinnern uns, daß sich alle rechnerisch festgestellten Spannungen und Dehnungen proportional zur gewählten spezifischen Belastung ändern würden.

Den Beispielen $\mathrm{X}$ und $\mathrm{X}_{f}$ ist der Boden aus Flußeisen zugrunde gelegt, der von Prof. v. Bach bezüglich Durchbiegen untersucht und in der Z. d. V. d. I. . I 899 S. 1585 in Fig. I und 2 abgebildet wurde. $(E=2100000 \mathrm{~kg} / \mathrm{qcm} ; m=10 / 3$.) Die Hauptrechnungsergebnisse sind in den Diagrammen Fig. 32 bis 43 aufgetragen.

\section{Diskussion der Rechnungsergebnisse.}

a) HinfluB der Randbedingungen »außen frei aufliegend und "außen eingespannt".

Die Beispiele I und II nach den Schnittfiguren 2 I and 22 wurden bereits eingehend erörtert. Die aus den Fig. I5 bis 18 bekannten Spannungen der beiden äußeren und der mittleren Faser sind in kleinerem Maßstab und als einfache Funktion des Abstandes $x$ von der Symmetrieachse in den Fig. 32 und 33 wiedergegeben in gleicher Weise wie in den Fig. 34 bis 43 für die übrigen Beispiele.

\section{b) Einfluß der Plattenwölbung.}

Die einschlägigen Zahlenbeispiele I, III, IV und $\mathrm{V}$ beziehen sich alle aul gußeiserne volle Platten mit einem äußeren Halbmesser $x_{a}=90 \mathrm{~cm}$ und einer gleichbleibenden Dicke $h=6 \mathrm{~cm}$ (s. Schnittskizzen 21, 23, 24 und 25). Die Fig. 32, 34, 35 und 36 geben ein Bild für den Verlauf der Spannungen in der mittleren und in den beiden äußersten Fasern einer jeden Platte.

In Fig. 44 sind die hauptsächlichsten Ergebnisse der 4 Rechnungsbeispiele in Funktion der Pfeilhöhe dargestellt, welche beträgt für die Platte

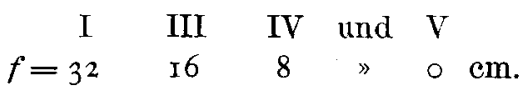

Die Krümmungshalbmesser dieser Platten betragen

Die Platte V ist »eben".

$$
\varrho=\mathrm{I} 43260 \quad 510 \Rightarrow \infty \mathrm{cm} .
$$

Von der Wölbung $f=32 \mathrm{~cm}$ ausgehend, nimmt die mittlere Radialspannung $\sigma_{r}$ mit abnehmender Plattenwölbung anfänglich schwach, dann immer mehr zu. Sinkt beispielsweise die Plattenwölbung " $f$ " von 32 auf I 6 und $8 \mathrm{~cm}$, so steigt die Spannung $\sigma_{r 0}$ von 258 auf 720 und $1085 \mathrm{~kg} / \mathrm{qcm}$ Druck.

Dieses Verhalten ist $z u$ vergleichen mit der Beanspruchung zweier nach Fig. 45 gegeneinander gestellter, gleich langer, gerader Stäbe. Bei gleichbleibender Last $P$, welche am gemeinsamen Gelenkpunkt $G$ angreift, wird die auf jede Stütze entfallende Komponente $N$ und damit die spezifische Normalspannung (Druck) in den Stützen mit abnehmender Pfeilhöhe immer größßer.

Die übrigen, in den vier Platten I, III, IV und V aúitretenden Spannungen sind entweder der Zahlentafel 8 oder dem Diagramm Fig. 44 zu entnehmen. Letzteres zeigt deutlich, wie die Höchstspannung mit zunehmender Wölbung abnimmt. Geben wir beispielsweise der Platte statt einer Pfeilhöhe von $8 \mathrm{~cm}$ eine solche von $32 \mathrm{~cm}$, so sinkt die größte Tangentialspannung in der Außenlaser und damit die in der Platte überhaupt auftretende größte Spannung vom Betrag $+3624 \mathrm{~kg} / \mathrm{qcm}$ auf $+\mathrm{I} 486 \mathrm{~kg} / \mathrm{qcm}$. Dabei steigt das Gewicht der Platte 
nur von 1065 auf I $80 \mathrm{~kg}$, also nur im Verhältnis I: I,II. Der geringe Mehraufwand an Gewicht von II vH ergibt eine Verringerung der Beanspruchung im Verbailtnis $2,44 \mathrm{zu}$ I oder lïßt eine im Verbäitnis I zu 2,44 gesteigerte Belastung zu. Der Vergleich der rechnerischen Ergebnisse von den Platten I und IV zeigt, wie sehr sich der geringe Materialmehraufwand lohnt.

Wie bereits weiter oben elwïhnt wurde, ist eine Platte nach Schema I im Betrieb auf $I_{5}$ at, statt wie in obiger Rechnung angenommen mit 20 at belastet. worden. Ihre rechnerisch höchste Beanspluchung betrug hierbei $1486 \times \frac{15}{20}$

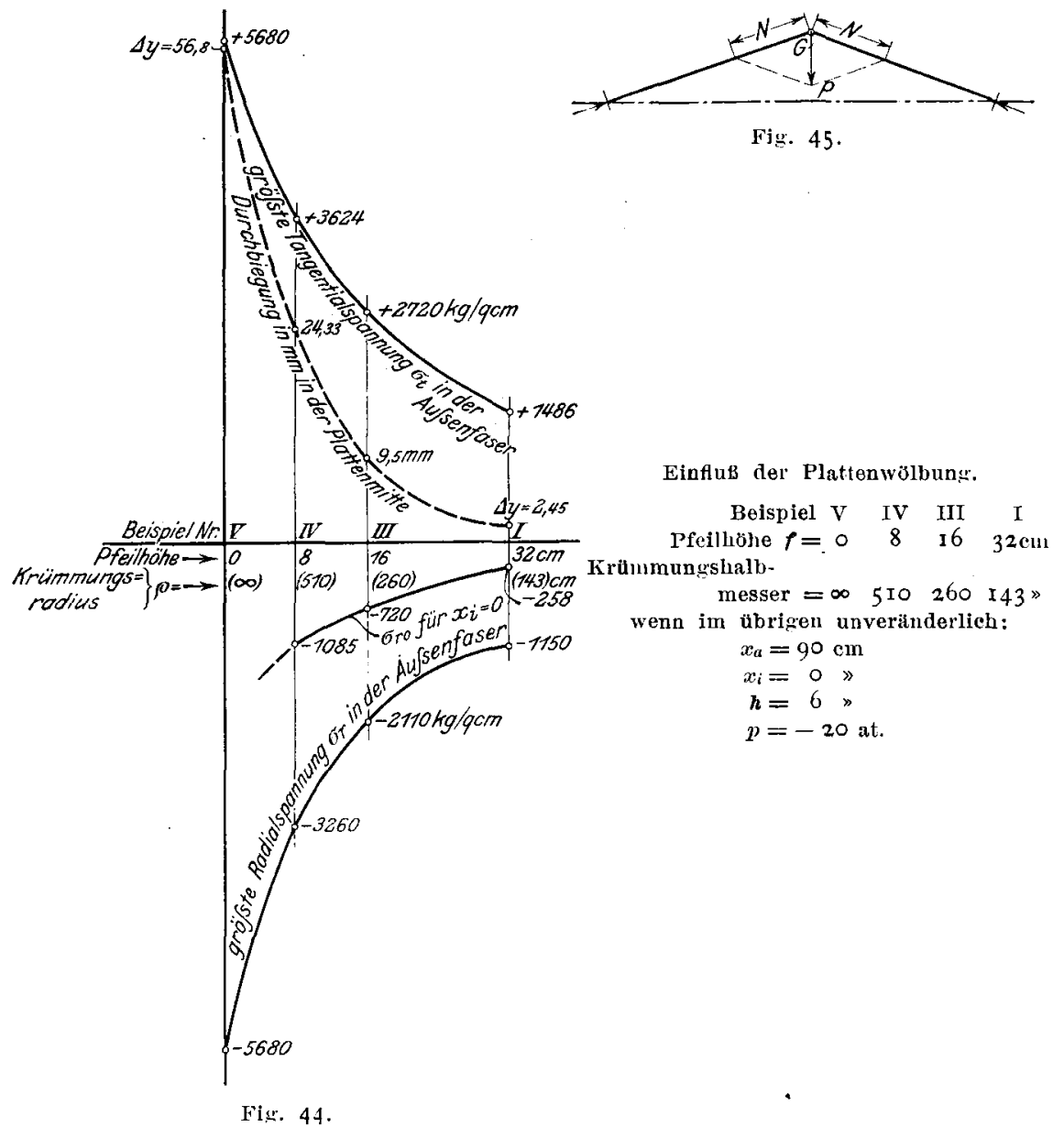

$=1056 \mathrm{~kg} / \mathrm{qcm}$, lag also für Gußeisen schon in ziemlich gefährlicher Nähe der Bruchgrenze. Jedenfalls hätte, wie die Beispiele III und IV zeigen, die Platte für diese hohe Belastung von $I_{5} \mathrm{~kg} / \mathrm{q} \mathrm{cm}$ nicht mit geringerer Wölbung (bei gleichbleibender Dicke) ausgeführt werden dürfen - von der Anwendung einer ebenen, frei aufliegenden Platte $V$ gar nicht zu reden.

c) Einfluß der Plattendicke $» / \ll$.

Die Platten . . . . . . . . . . . . . . . . I

nach den Schnittiguren $\mathrm{Nr}$....................

unterscheiden sich nur durch ihre stetsgleichen Dicken 
I VI

welche sich verhalten wie . . . . . . . . . . $\quad$ I $\quad$ : I, 5 ,

deren Quaảrate sich verhalten wie . . . . . . I I : 2,25 .

Laut Zahlentafel 8 und gemäß Fig. . . . . . . . 32 bezw. 37 treten in diesen beiden Platten iolgende Spannungen auf:

I) Radial- gleich Tangentialspannungen in der Mittelfaser und in der Plattenmitte $\left.\sigma_{r 0}\right|_{x=0}=. \cdot-25^{8}-212 \mathrm{~kg} / \mathrm{qcm}$.

Diese verhalten sich wie . . . . . . . . . . $1,22 \quad:$ I, also nicht, wie man etwa vermuten könnte, wie die Plattendicken . . . . . . . . . . . .

2) Tangentialspannungen in der Mittelfaser am Außenrand $\sigma_{10}{ }_{x=90}$. . . . . . . . . . . . . $+1277+620$,

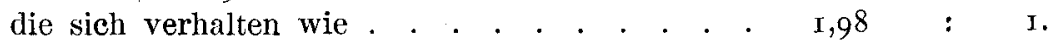

3) Größte Tangentialspannung in der Außenfaser am $\ddot{a} u ß e r e n$ Rand $\sigma_{t} \frac{h}{2}: x=90$.. . . . . . . . $+\mathrm{r}_{4} 86+810 \mathrm{~kg} / \mathrm{qem}$, deren Verhältnis gleich. . . . . . . . . . $\mathrm{I}, 82 \quad$ : $\mathrm{I}$.

4) Größte Radialspannung in einer der Außenfasern . -- II50 $-620 \mathrm{~kg} / \mathrm{qcm}$. Sie verhalten sich wie. . . . . . . . . . . $\mathbf{1}, 7 \mathbf{2}$ : $\mathbf{I}$

Keine der unter 2) bis 4) einander gegenübergestellten Faserspannungen der Beispiele I und VI verhalten sich - wie man hätte vermuten können umgekehrt wie die Quadrate der Plattendicke (2,25: I) und wie dies bei ebenen Platten der Fall wäre. Das Verhältnis ist kleiner. In sonst gleichartig gebauten gewölbten Platten sinkt demnach die Beanspruchung in einem geringeren Maße als umgekehrt zum Quadrat der Dicke, wenn man von einer gegebenen Platte zu einer dickeren übergeht.

\section{d) Einflub des aiußeren Halbmessers $x_{a}$ ".}

Die Platten der beiden Rechnungsbeispiele . . . . I und VII haben gemä $B$ Schnittskizzen . . . . . . . . . 21 bezw. 27 bei sonst gleichbleibenden Abmessungen einen Außenhalbmesser $x_{a}$. . . . . . . . . . . . . . $9060 \mathrm{~cm}$. Diese Halbmesser verhalten sich wie. . . . . . . I,5 : I. Ihre Quadrate verhalten sich wie . . . . . . . 2,25 : I. Laut Diagramme ... . . . . . . . . . . . . $32 \quad 3^{2}$ treten in diesen zwei Platten folgende Spannungen auf:

I) Größte Radial- resp. 'Tangentialspannung in der

Symmetrieachse, wo $x_{i}=0$. . . . . . . $-36_{5}-60_{5} \mathrm{~kg} / \mathrm{qcm}$, sie verhalten sich wie. . . . . . . . . . . I : $\mathrm{I}, 666$.

2) Größte Radialspannung . . . . . . . . - II $50-950 \mathrm{~kg} / \mathrm{qcm}$, ihr Verhältnis ist. . . . . . . . . . . . $\mathrm{I}, 2 \quad$ : $\mathrm{I}$.

3) Größte Tangentialspannung . . . . . . . $+1486+1 \mathrm{I} 79 \mathrm{~kg} / \mathrm{qcm}$, deren Verbältnis . . . . . . . . . . . . . $\mathrm{I}, 26 \quad:$ I.

Irgend eine Gesetzmäßigkeit für die Abhängigkeit der Normalspannungen von dem einfachen Plattendurchmesser oder von dessen Quadrat scheint hier also nicht zu herrschen. Im Gegensatz zu ebenen Platten, wo die Spannungen sich wie die Quadrate der Plattendurchmesser verhalten, nehmen die unter I) 
genannten Spannungen mit kleiner werdendem Außenhalbmesser wider Erwarten sogar noch zu. Die unter 2) und 3) aufgefiuhrten Spannungen nehmen nicht einmal im umgekebrten Verhältnis der einfachen Außenhalbmesser ab, geschweige denn im umgekehrten Verhältnis von deren Quadraten.

Daß für den Zusammenhang der Beispiele VI und VII sich keine Gesetzmäßigkeit aus den Spannungsdiagrammen 37 und $3^{8}$ herauslesen lißßt, trotzdem die Durchmesser 90 bezw. $60 \mathrm{~cm}$ und die Dicken $h=9$ bezw. $6 \mathrm{~cm}$ ähnlich sind, rührt davon her, dal. die Krümmungshalbmesser nicht auch im gleichen Verhältnis zueinander geändert, sondern einander gleich belassen wurden, nämlich $\varrho=143 \mathrm{~cm}$.

Erst die Platte VIII steht in allen Abmessungen im gleichen Verhältnis zur Platte VI, nämlich 3:2. Es konnte daher erwartet werden, daß ähnlich gelegene Punkte genau gleiche Spannungen aufweisen. In Wirklichkeit zeigen die Ausrechnungen für Beispiel VI eine größte Spannung von $(-620) \mathrm{kg} / \mathrm{qcm}$, für Beispiel VIII eine solche von $(-550) \mathrm{kg} / \mathrm{qcm}$. Daß diese Ergebnisse wie auch die Spannungsdiagramme 37 und 39 überhaupt nicht besser übereinstimmen, liegt an zwei Gründen. Fürs erste hätten bei beiden Zahlenbeispielen weitere Durchrechnungen gemacht werden sollen, um den wahren Werten noch näher zu kommen. Sodann kommt bei den in der Mitte vollen Platten eine Unsicherheit dadurch hinein, daß man die Rechnung nicht mit $x=0$ beginnen kann. So wurde bei Beispiel VI mit $x=1 \circ \mathrm{cm}$, bei Beispiel VIII mit $x=8 \mathrm{~cm}$ begonnen, und es mußten für diese Halbmesser Annahmen getroffen werden, nicht nur für $\frac{d \psi}{d x}$, sondern auch noch für $\psi$.

Wenn man zuverlißlicher rechnen will, muß man mit kleinerem $x$ beginnen und kleinere Intervalle wählen. Dies ist jedoch recht zeitraubend. Mit Rücksicht darauf, daß ich mir die Berechnung so vieler Beispiele zur Aufgabe gestellt, habe ich obige Ungenauigkeit in den Kauf genommen. Für einen einzelnen Fall würde man dieser einen Berechnung noch mehr Zeit opfern müssen.

\section{e) Einfluß der Bohrung und der versteifenden Nabe.}

Hierüber gibt ein Vergleich der Zahlenbeispiele I und IX Auskunft. Die Platte IX ist dargestellt in Fig. 29 im Maßstab $r: 45$ und in Fig. 46 im Maßstab $I: 20$ der Fig. I4, welche ein Bild der zuerst betrachteten Platte I ist. Die Platte IX unterscheidet sich von Platte I nur dadurch, daß in ihrer Mitte eine Bohrung vom Halbmesser $x_{i}=I_{5} \mathrm{~cm}$ und daß das durch diese Bohrung entfernte

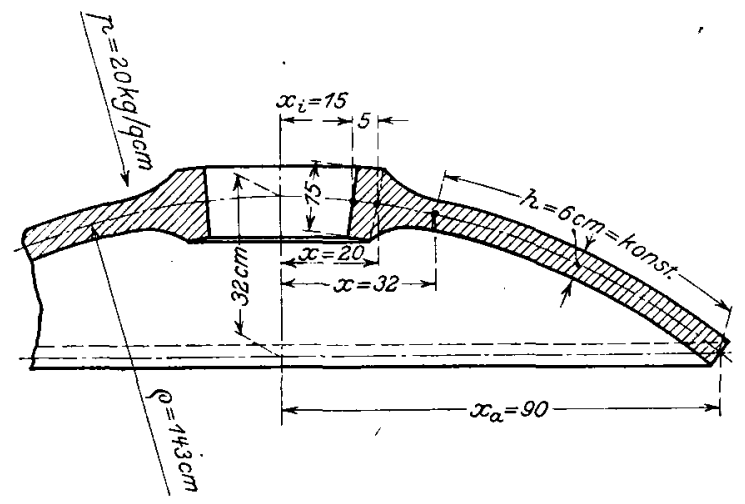

Fig. 46. Platte IX. I :20. 
Material ersetzt ist oder vielmehr sein soll durch eine Nabe von ${ }_{5} \mathrm{~cm}$ axialer Länge und $5 \mathrm{~cm}$ Dicke; diese Nabe geht allmählich auf die ursprüngliche Plattendicke $h=6 \mathrm{~cm}$ über. Von da ab bis an den Außenrand $\left(x_{a}=90 \mathrm{~cm}\right)$ ist die stets gleiche Plattendicke $h=6 \mathrm{~cm}$, wie in Beispiel I. Diese neue Platte soll außen ebenfalls frei aufliegen und durchgehend von der konvexen Seite her mit $20 \mathrm{~kg} / \mathrm{qcm}$ belastet sein.

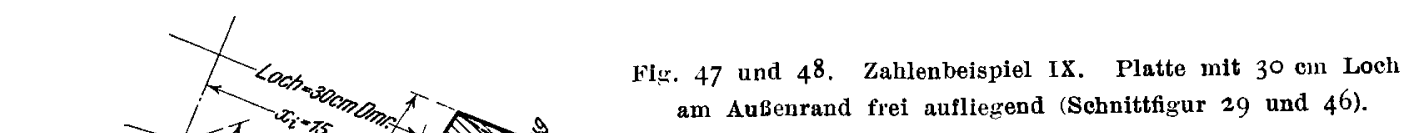

Fig. 47. Radialspannung 0 , in $\mathbf{k} g / q \mathrm{~cm}$.

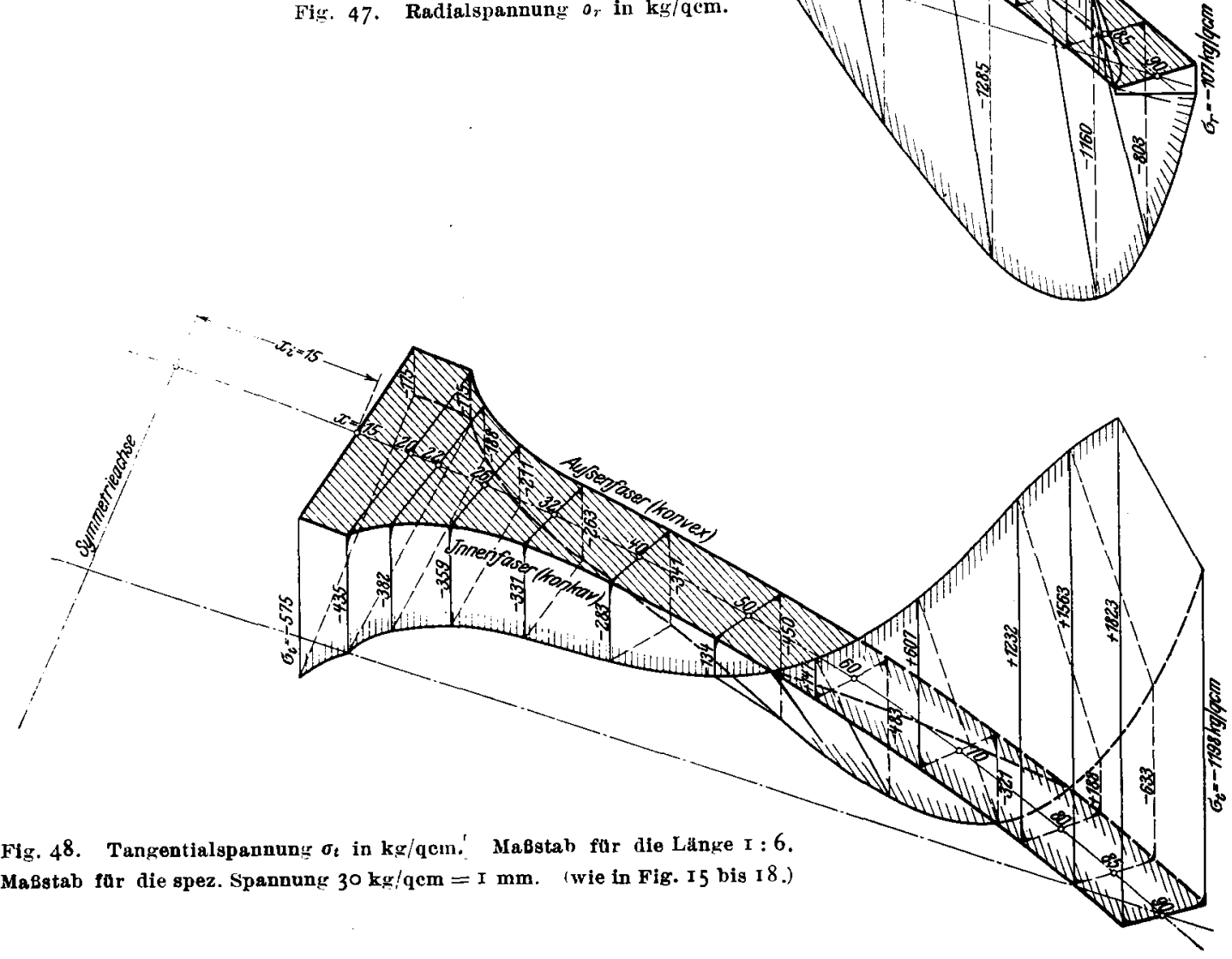


Die Fig. 40 zeigt im gleichen Maßstab, wie Fig. 32 für I'latte I den Verlauf der Spannungen in den beiden Außen- und in der Mittelfaser. Desgleichen sind in Fig. 47 in axonometrischer Weise die Radialspaunungen und in Fig. 48 die Tangentialspannungen dargestellt, wie in den Fig. 15 und r6 für Platte I. Diese Diagramme zeigen, dals wir die Abmessungen der Nabe ziemlich gut getroffen haben. Die höchste auftretende Spannung, die Tangentialspannung $\sigma_{t}$ in der konkaven Außenfaser am Außenrand, beträgt $(+1822)$ statt in Platte I $(+\mathrm{I} 486 \mathrm{~kg} / \mathrm{qcm})$. Die höchste Radialspannung beträgt (- I $300 \mathrm{~kg} / \mathrm{qcm}$ ) gegenüber $(-\mathrm{Ir} 50 \mathrm{~kg} / \mathrm{qcm})$. Die beiden Spannungsdiagramme 32 und 40 reigen in den äußeren Hialften der Platten I und IX einen völlig gleichartigen Verlauf. eingetragen.

Die Verschiebungen der Meridianmittelfaser sind in die Fig. I9 und 20

\section{i) Einfluß der Vordickung des PIattenrandes.}

Um diesen Einfluß festzustellen, wurde als Beispiel $I_{v}$ [verdickt] eine in Fig. $3 \circ \mathrm{im} \mathrm{Maßstab} \mathrm{I}: 45$, in Fig. $49 \mathrm{im}$ gleichen Maßstab wie Platte 1 in Fig. I4 dargestellte Platte gerechnet, welche sich von Platte IX dadurch unterscheidet, daß ihre Dicke nur zwischen den Halbmessern $x=32$ und $x=65$ unverändert $=6 \mathrm{~cm}$ ist und daß sie von diesem Halbmesser ab gegen den Außenrand hin allmählich bis zum Betrag $h_{a}=9 \mathrm{~cm}$ ansteigt. Am Rand ist sie wie die Platten $1 X$ und I frei aufliegend gedacht (s. die Fig. I4, 49 und 46).

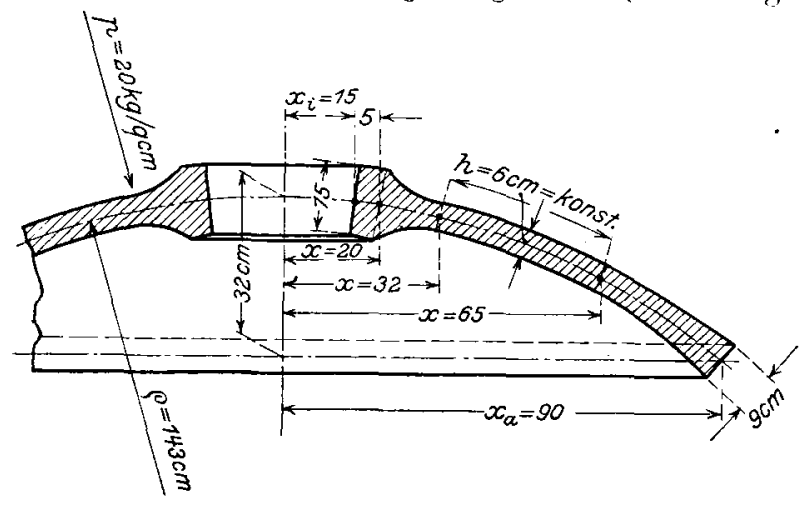

Fig. 49. Platte IX $\mathbf{X}_{*}$ I : 20 .

Durch die Verdickung des Außenrandes nach Fig. 49 gegenüber Fig. 46 ist laut Diagramm 4I gegenüber Diagramm Fig. 40 die höchste Spannung gesunken vom Betrag $(+\mathrm{I} 822)$ auf den Betrag $(+1573)$ und die Durehbiegung in der Mitte vom Betrag $(-2,78) \mathrm{mm}$ auf den Wert $(-2,30) \mathrm{mm}$. In Diagramm ${ }_{4 \mathrm{I}}$ sind in dünnen Linien die Werte aus dem Spannungsdiagramm 40 wiederholt, so daß die Abweichung leicht ersichtlich ist. Die Platte IX $\mathrm{I}_{v}$ ist bezüglich Beanspruchung von der Platte I nur unwesentlich versehieden. Wir haben also tatsächlich den an sich schädlichen und sonst so gefürchteten Einfluß der Bohrung in der Mitte durch die Ausbildung der Nabe und die Verstärkung des Plattenrandes ausgeglichen.

Vergleichshalber sind von den Rechnungsbeispielen

$$
\begin{aligned}
& \text { I nach Fig. I4 bezw. 2I (frei aufliegend) } \\
& \text { II " } " 22 \text { (eingespannt) } \\
& \text { IX } " ~ " 46 \Rightarrow 29 \text { (frei aufliegend) } \\
& \mathrm{IX}_{v} \text { » }, 49 \text { " } 30 \text { " }
\end{aligned}
$$


jo in einem Diagramm vereinigt und als Eunktion des Abstandes $x$ von der Symmetrieachse aufgetragen:

In Fig. 50 die Durchbiegung $J_{y}$ der Meridian-Mitteliaser.

In Fig. $5^{\mathrm{I}}$ die Aenderung $J x$ der Abstande $x$ der einzelnen Punkte der Meridian-Mittelfaser von der Symmetrieachse.

In Fig. $5^{2}$ die Aenderung $\psi$, die der zwischen dem Krümmungshalbmesser und der Symmetrieachse gelegene Winkel $\boldsymbol{p}$ erleidet.

Die Werte der Platten I, IX und IX, weichen nicht sehr voneinander ab, dagegen verhält sich die Platte II ganz anders, und man sieht daraus nochmals den guinstigen Einfluß des »Eingespanntseins". Wie Fig. 52 zeigt, drehen sich alle Querschnitte des inneren Teiles der Platten im Sinne der Winkelvergrößerung,

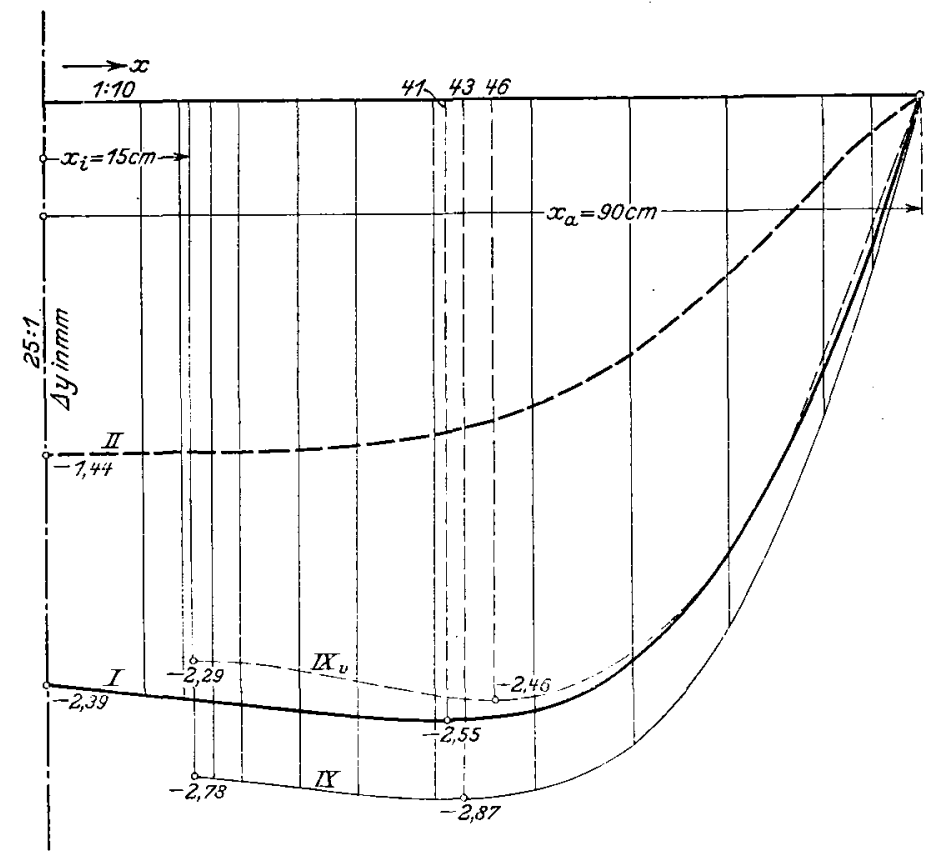

Fìr. 50. Durchbiegung $\Delta y$ in Funktion des Abstandes $x$ von der Symmetrieachse für die Zahlenbeispiele I, II, IX, IXr.

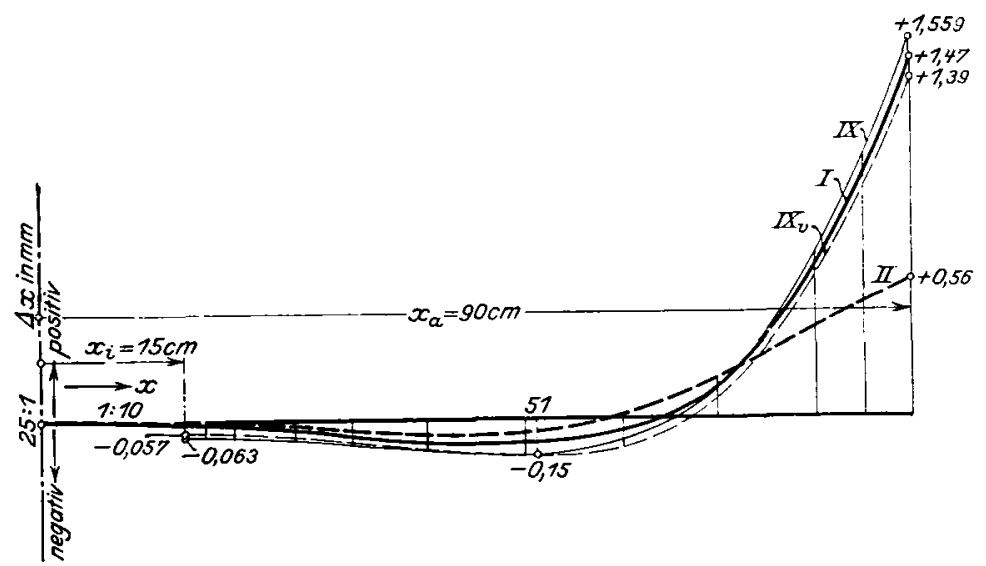

Fị. 5x. Aenderung $d x$ in Funktion des Abstandes $x$ von der Symmetrietchse für die Zahlenbeispiele I, II, IX, IX 
d. h. die Punkte der konvexen Außenfaser entfernen sich, diejenigen der konkaven Innenfaser nähern sich der Symmetrieachse. In den weiter außen gelegenen Querschnitten ist das Umgekehrte der Fall. Zwischendrin liegt ein Inflexionspunkt, wie ihn Prof. Dr. Stodola für den kegelförmigen Boden berechnet hat ${ }^{1}$ ). Für die am Rand eingespannte Platte II liegt er bei $x \sim 20 \mathrm{~cm}$,

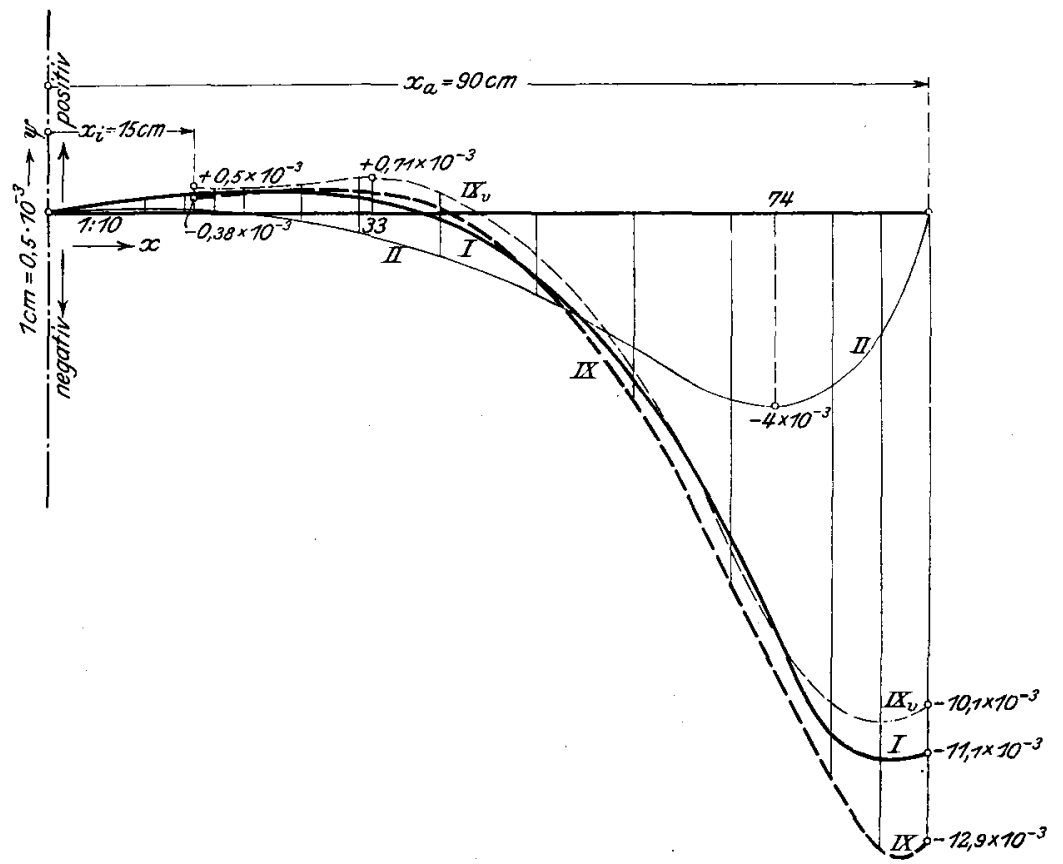

Fig. 52. Winkeländerung $\psi$ in Funktion des Abstandes $x$ von der Symmetrieachse für die Zahlenbeispiele I, II, IX, IX $\mathbf{X}_{v}$.

fiur die drei am Rand frei aufliegenden Platten I, IX und $\mathrm{IX}_{v}$ nahe beieinander, und zwar bei $x \sim 40 \mathrm{~cm}$.

Wie eine aus der Grundform I entwickelte Platte mit Bohrung jedoch ohne versteifende Nabe beansprucht wäre und sich durchbiegen würde, und wie die Beanspruchungen und Durchbiegungeu von der Größe der Bohrụng abhängig sind - dies zu untersuchen, mag einer besonderen Arbeit vorbehalten bleiben.

\section{Die Biegungsmomente der inneren Spannungen in Abhängigkeit vom Halbmesser $x$.}

Bei der Berechnung des Ausdruckes $\left(\frac{d \psi}{d x}\right)$ stießen wir unter anderen auf den Wert $\boldsymbol{M}_{\boldsymbol{\sigma}_{r}}$, das ist das Moment der auf die Begrenzungsfläche $C D E \boldsymbol{F}$ des in Fig. 3 dargestellten Plattenelementes wirkenden Normalspannungen $\sigma_{r}$. Wir fanden als Gl. (20):

$$
M_{\sigma_{r}}=d \alpha c x_{12}^{h^{3}} \cos \varphi\left[m \frac{d \psi}{d x}+\begin{array}{l}
\psi \\
x
\end{array}\right] .
$$

Dividieren wir diesen Ausdruck durch die mittlere Breite $(x d \alpha)$ der Fläche $C D E F$, so erhalten wir den auf je einen Zentimeter des mittleren Parallelkreisumfanges entfallenden Anteil des Momentes $\boldsymbol{M}_{\boldsymbol{\sigma}_{r}}$, nämlich:

$$
M_{\boldsymbol{\sigma}_{r}}{ }^{\prime}=\frac{\boldsymbol{M}_{\boldsymbol{\sigma}_{r}}}{(\boldsymbol{x} d \boldsymbol{a})}=h^{3} \cos \phi\left[m \frac{d \psi}{d \boldsymbol{x}}+\frac{\psi}{x}\right]_{\mathrm{I} 2}^{c} \text {. . . . (48). }
$$

1) S. Stodola: "Die Dampfturbinen", IV. Auflage S. 602. 
Wir können diesen Wert nennen »das spezifische Moment der Normalspannungen $\sigma_{r} \ll$. Seine Maßeinheit ist $\mathrm{kgcm} / \mathrm{cm}$. In unserer abgekül'zten Ziffernsprache lautet die Formel (vergl. Zahlentafel 3 und 7 ):

$$
M_{\sigma_{r}^{\prime}}^{\prime}=(44)(105) \frac{c}{12} \text {. . . . . . . }(48 \mathrm{a}) \text {. }
$$

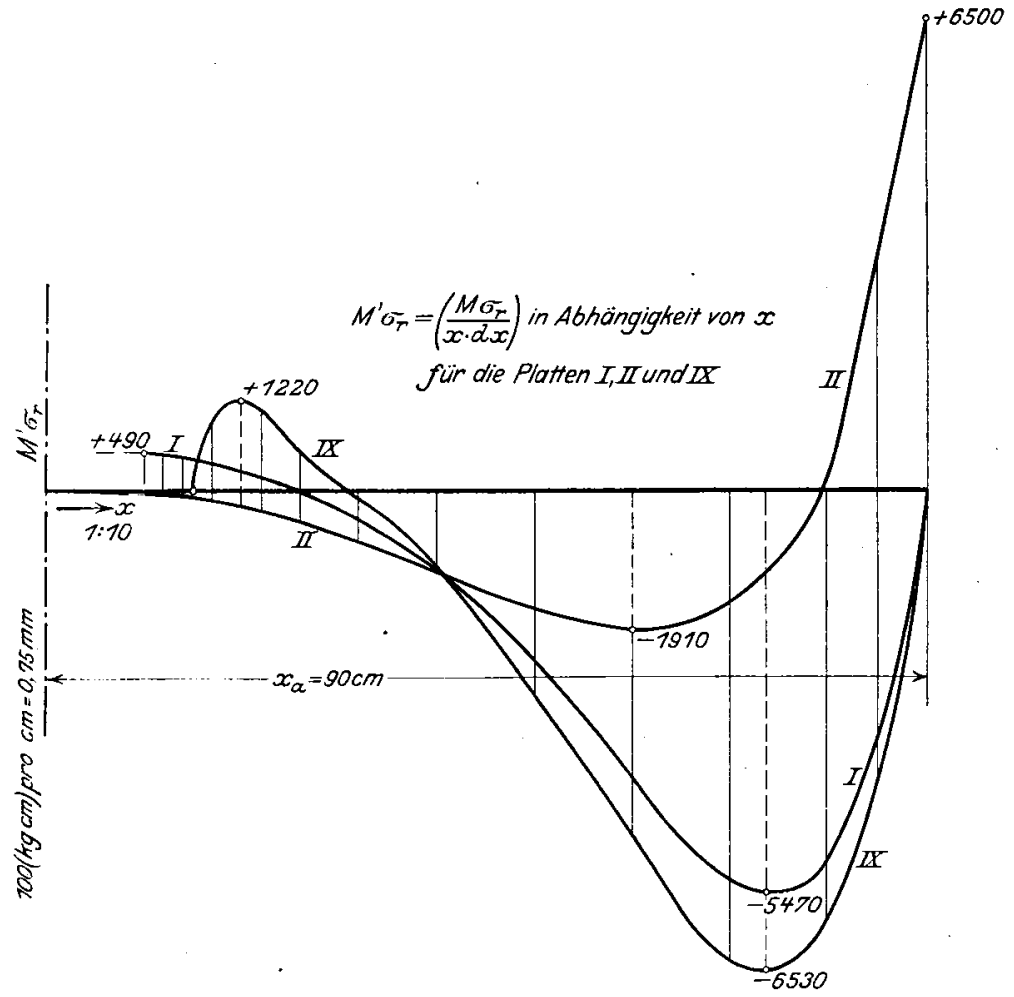

Fig. 53. Maßstab für die Ordinaten: $100 \mathrm{kgrm} / \mathrm{cm}=0,75 \mathrm{~mm}$.

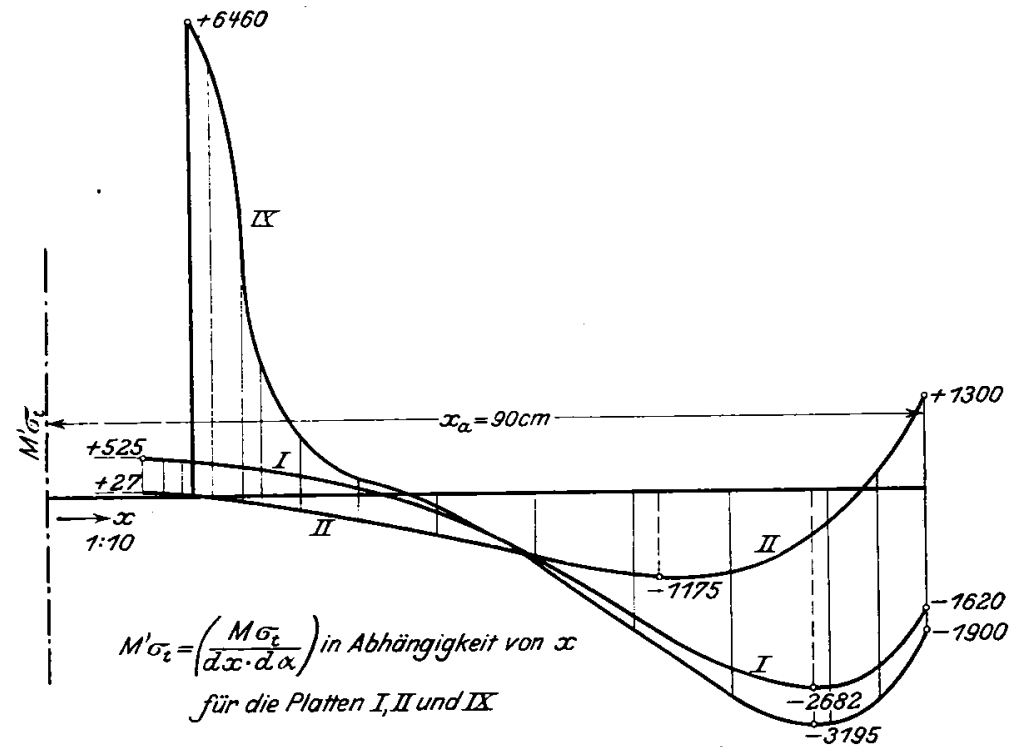

Fig. 54. Maßstab für die Ordinaten: $100 \mathrm{kgem} / \mathrm{cm}=0,75 \mathrm{~mm}$. 
In ganz gleicher Weise läbt sich für das auf das Plattenelement $C$ bis $K$, Fig. 3 bezw. 9, wirkende resultierende Moment.$I_{\sigma_{t}}$, welches von den Tangentialspannungen $\sigma_{t}$ herrührt, aus der (il. (24) das "spezifische« Ioment $M_{\sigma_{t}}{ }^{\prime}$ ableiten:

$$
\begin{aligned}
& M_{n_{t}^{\prime}}={ }_{d x d x t}^{M I_{\sigma_{t}}}=\left(h^{3} \cos \varphi\right)\left[\frac{d \psi}{d x}+m \frac{y^{\prime}}{x}\right] \frac{c}{12} \cdot \text {. . . (49). }
\end{aligned}
$$

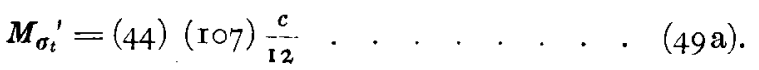

Beispielshalber wurden die in den Gl. (48) und (49) definierten Werte für eine größere Anzahl der Werte von $x$ ausgerechnet für die Platten I, II und IX und in den Diagrammen Fig. 53 bezw. 54 bildlich dargestellt in Funktion von $x$.

\section{Die für eine gegebene Platte erforderlichen Durchrechnungen.}

Um zu zeigen, wie sich bei den einzelnen Durchrechnungen für verschiedene Annahmen die Endergebnisse stellen, und wie manche Durchrechnungen überhaupt durchgeführt werden müssen, greifen wir das Zahlenbeispiel IX heraus. Zur Berechnung der in Fig. $4^{6}$ dargestellten Platte wurden fünf verschiedene Annahmen gemacht mit dem in nachstebender Zahlentafel ersichtlichen Eriolg:

$$
\begin{aligned}
& \text { Durchrechnung . . . . . Nr. 91 } 92 \quad 93 \quad 94 \quad 95 \\
& \text { Annahme für } x_{i}=\mathrm{I} 5 \mathrm{~cm}\left(\text { wo } \sigma_{r 0}=0\right) \\
& \sigma_{t 0 i}=. \cdot \cdot \cdot-320-630-400-330-375 \mathrm{~kg} / \mathrm{qcm} \\
& \psi_{i}=. \text {. . . }+190+225-\mathrm{I}_{50}+285+385 \cdot \mathrm{10}^{-1 i}
\end{aligned}
$$

Endergebnisse für $x_{a}=90 \mathrm{~cm}$.

a) Spannungsprobe:

$$
\begin{aligned}
& \sigma_{r 0 a}=\cdot \cdot \cdot \cdot-\mathrm{IO}_{4}+603+\mathrm{r}_{3} 8-\mathrm{I}_{2} 6-63 \mathrm{~kg} / \mathrm{qem} \\
& \text { statt nach Gl. (32a) - } 92-92-92-92-92 \% \\
& \text { Fehlbetrag = - } 12+695+230-34+29 \text { » }
\end{aligned}
$$

b) Winkelprobe nach Gl. (33).

$$
\begin{array}{lccccc}
{\left[m \frac{d \psi}{d x}+\frac{\psi}{x}\right]=} & +899+8^{\prime} 792+7^{\prime} 029 & +447 & -100 \cdot 10^{-6} \\
\text { statt . . . . . . . . . } & 0 & \circ & 0 & \circ & \circ
\end{array}
$$

Wie diese Zusammenstellung zeigt, hätten eigentlich noch mehr Durchrechnungen erfolgen sollen, um den in den Gl. (32a) und (33) gestellten Randbedingungen näher zu kommen. Doch ist schon bei der Durchrechnung 95 die Annäherung für die Praxis durchaus hinreichend. (Beim Aufzeichnen des Spannungsdiagrammes 34 wurden die Kurven für die Radialspannungen $\sigma_{r}$ so gedreht, daß sie bei $x_{n}=90 \mathrm{~cm}$ durch den Punkt $(-92 \mathrm{~kg} / \mathrm{cm})$ hindurchgehen.) -Bei anderen Zahlenbeispielen waren allerdings weit mehr Durchrechnungen erforderlich als nur fünf. Um folgenschwere Fehler zu verhüten, empfiehlt sich das fortlaufende graphische Aufzeichnen der Zwischwerte.

Als weiteres Anwendungsbeispiel wurde endlich die Platte nachgerechnet, die seinerzeit durch Prof. v. Bach untersucht worden ist, und deren Prüfungsergebnisse in der Z. d. V. d. I. I 899 S. 1586 veröffentlicht sind. Die Platte $A$ bestand aus Flußeisen $\left(E=2100000 \mathrm{~kg} / \mathrm{qcm} ; m={ }^{10} / 3\right)$. Unsere Schnittskizze Nr. $3 \mathrm{I}$ ist der Fig. I ans Z. d. V. d. I. 1899 S. 1585 nachgebildet. Wir führen die Rechnung nur durch bis zum Beginn der Krempe, das ist bis zum Halbmesser $x=30 \mathrm{~cm}$ und nehmen an, daß die Platte daselbst »eingespannt «, 
und daß sie auf der konkaven Seite gleichmäßig mit $p=+\mathrm{I} 6$ at (statt wie in den bisherigen Beispielen mit $p=-20$ at) belastet sei. "Eingespannt" soll heißen, daß der Rand sich nicht verdrehen kann. Dieses "Eingespanntsein * wollen wir im Rechnungsbeispiel $X$ so auffassen, daß der Außenrand in Richtung senkrecht zur Symmetrieachse beliebig nachgeben kann, während in Beispiel X der Meridianpunkt des Außenrandes so festgehalten gedacht werde, daß er sich in Richtung senkrecht zur Symmetrieachse kaum bewegt, daß er also nahezu "fix " sei. Daher die Bezeichnung " $\mathbf{X}_{f}$. Die Spannungen des Rechnungsbeispieles $\mathrm{X}$ sind im Diagramm 42 , diejenigen des Beispieles $X_{f}$ im Diagramm 43 aufgetragen. (Ihre Malsstäbe sind jedoch verschieden von denjenigen der Diagramme 32 bis $4 \mathrm{I}$.)

Das Spannungsdiagramm 43 zeigt mit seinen viel kleineren Ordinaten als im Diagramm 42 den günstigen Einfluß dieses $\gg$ Festhaltens « des Plattenrandes in Richtung senkrecht zur Symmetrieachse. Die Höchstspannung ist weit unter die Hälite gesunken gegenüber derjenigen in der Platte $\mathrm{X}$, deren Außenquerschnitt sich zwar auch nicht drehen, wohl aber radial verschieben kann. Dementsprechend ist auch die rechnerische Durchbiegung in der Plattenmitte viel kleiner, nämlich $0,5 \mathrm{I}$ statt 2,00 mm (siehe Zahlentafel 8).

v. Bach hat bei I6 at Belastung in der Mitte gegenüber dem Beginn der Krempe, d. i. gegenüber den Meßpunkten 4, 16,28 , 40 eive Durchbiegung von $\mathrm{I}, 25-0, \mathrm{I} 7=\mathrm{I}, 08 \mathrm{~mm}$ gemessen. Dieser Wert liegt zwischen den in unseren Beispielen $X$ und $X_{f}$ berechneten Werten 2,0 bezw. 0,5 I mm. Leider fehlt in der v. Bachschen Veröffentlichung die Angabe, um wieviel sich der Normalabstand der Meßpunkte 4, 16, 28 and 40 von der Symmetrieachse geändert hat. Es ist in der Zusammenstellung 7 S. I 586 nur gesagt, daß der Außenrand der Krempe, d. h. daß die Melspunkte 49 bis $5^{1}$ bei $p=16$ at im Mittel um $0,099 \mathrm{~mm}$ nach innen gerückt sind. Es ist jedoch anzunehmen, daß die Meßpunkte 4, I6, 28, to sich eher noch mehr nach innen verschoben haben. Unsere Beispiele $X$ und $\mathrm{X}_{i}$ ergeben eine Verkürzung des Außenhalbmessees $\left(x_{a}=30 \mathrm{~cm}\right)$ von 0,33 bezw. $0,04 \mathrm{~mm}$. Damit ist nachgewiesen, daß der von Bach untersuchte Boden bezüglich wirklicher Beanspruchung ein Mittelding zwischen unseren beiden rechnerisch untersuchten Grenzfällen $\mathrm{X}$ und $\mathrm{X}_{f}$ ist. Er wird also bei einer spezifischen Belastung von $I 6$ at eine Höchstbeanspruchung erfahren, die zwischen $+453^{\circ}$ von $F$ all $\mathrm{X}$ und $+199 \circ \mathrm{kg} / \mathrm{qcm}$ von $\mathrm{Fall} \mathrm{X}_{j}$ liegt. Vermutlich liegt diese wirkliche höchste Beanspruchung nahe bei $3000 \mathrm{~kg} / \mathrm{qcm}$.

Dieser Vergleich unserer Rechnungsergebnisse mit den Bachschen Meßergebnissen liefert den erwarteten Beweis für die praktische Verwendbarkeit vorliegenden Rechnungsverfahrens.

Alle Beispiele mit Ausnahme desjenigen Nr. V, d. i. der ebenen Platte, zeigen, daß der höchst beanspruchte Querschnitt sich außen am Rand der Hauptwölbung, oder wenigstens in dessen Nähe befindet, und tatsächlich sind auch wohl die meisten Brüche an Kesselböden von dieser Stelle, d. i. vom Uebergang zur Krempe, ausgegangeu.

Vorstehende Rechnungen, insbesondere diejenigen $\mathrm{Mr}$. I und $\mathrm{IX}_{v}$, sollen in erster Linie Beispiele für die Anwendbarkeit des eingangs theoretisch hergeleiteten Rechnungsverfahrens sein. Gleichzeitig geben sie ein sehr deutliches Bild der Abhängigkeit der Beanspruchung und der Durchbiegung einer Platte von ihrer Form und ihrer Unterstiutzungsart.

Ein großer Vorteil der hier angewendeten Rechnungsweise mit kleinen Differenzen liegt darin, daß für die Erzielung einer Rechnungsmöglichkeit weit 
weniger Einschränkungen gemacht werden müssen, als in den vorveröffentlichten Vorschlägen. Schüle mußte in seinem Aufsatz in "Dinglers Polyt. Journal " vom Io. Oktober I 900 voraussetzen, daß der Boden in der Mitte voll sei, überall gleiche Krümmung und Dicke habe. Von diesen drei Hauptbedingungen konnte Fankhausen für seine in der "Zeitschr. f. d. gesamte Turbinenwesen" vom Jahr I9I I S. 449 niedergelegte Arbeit die erste, aber auch nur die erste fallen lassen, mußte dagegen daran festhalten, daß die Platte ein Kugelboden von stets gleicher Dicke sei ${ }^{1}$ ). Und trotz dieser im Grunde doch recht großen und für die Anwendung in der Praxis hinderlichen Einschränkung sind Fankhausens Gleichungen nicht einfach zu nennen ${ }^{2}$ ).

Vorliegendes Verfahren gestattet die Ausrechnung von ungeteilten Böden mit

a) veränderlicher Dicke,

b) veränderlichem Wölbungshalbmesser,

c) Bohrung in der Mitte.

Als ein solch allgemeines Beispiel haben wir deshalb den Boden Nr. IX nach Schnittfigur Nr. $3 \circ$ bezw. Nr. 49 gewählt.

1) Vergl. Zeitschr. f. d. ges. Turbinenwesen« I9II S. $45^{\circ}$ Zeile 5 und S. 474 oben.

y) Sighe z. B. dessen Gl. (27). 